\title{
Lectures on Loop Quantum Gravity
}

\author{
T. Thiemann ${ }^{1}$ \\ MPI f. Gravitationsphysik, Albert-Einstein-Institut, \\ Am Mühlenberg 1, 14476 Golm near Potsdam, Germany
}

Preprint AEI-2002-087

${ }^{1}$ thiemann@aei-potsdam.mpg.de 


\begin{abstract}
Quantum General Relativity (QGR), sometimes called Loop Quantum Gravity, has matured over the past fifteen years to a mathematically rigorous candidate quantum field theory of the gravitational field. The features that distinguish it from other quantum gravity theories are 1) background independence and 2) minimality of structures.

Background independence means that this is a non-perturbative approach in which one does not perturb around a given, distinguished, classical background metric, rather arbitrary fluctuations are allowed, thus precisely encoding the quantum version of Einstein's radical perception that gravity is geometry.

Minimality here means that one explores the logical consequences of bringing together the two fundamental principles of modern physics, namely general covariance and quantum theory, without adding any experimentally unverified additional structures such as extra dimensions, extra symmetries or extra particle content beyond the standard model. While this is a very conservative approach and thus maybe not very attractive to many researchers, it has the advantage that pushing the theory to its logical frontiers will undoubtedly either result in a successful theory or derive exactly which extra structures are required, if necessary. Or put even more radically, it may show which basic principles of physics have to be given up and must be replaced by more fundamental ones.

QGR therefore is, by definition, not a unified theory of all interactions in the standard sense since such a theory would require a new symmetry principle. However, it unifies all presently known interactions in a new sense by quantum mechanically implementing their common symmetry group, the four-dimensional diffeomorphism group, which is almost completely broken in perturbative approaches.
\end{abstract}




\section{Contents}

\begin{tabular}{ll|l}
\hline Motivation and Introduction & 5
\end{tabular}

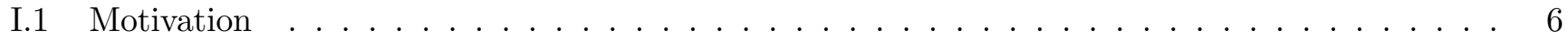

I.1.1 Why Quantum Gravity in the 21'stCentury ? . . . . . . . . . . . . . . . . . . . 6

I.1.2 The Role of Background Independence . . . . . . . . . . . . . . . . . . . . . . 9

I.2 $\quad$ Introduction: Classical Canonical Formulation of General Relativity $\ldots \ldots \ldots$. . . . . . . . . 15

$[.2 .1$ The ADM Formulation $\ldots \ldots \ldots \ldots \ldots \ldots \ldots \ldots$

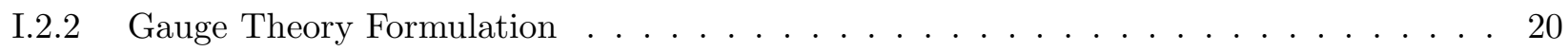

I.3 Canonical Quantization Programme for Theories with Constraints . . . . . . . . . . . . . . . 22

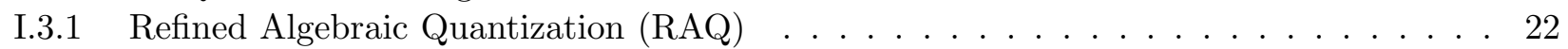

I.3.2 $\quad$ Selected Examples with First Class Constraints . . . . . . . . . . . . . . . . . . 25

II Mathematical and Physical Foundations of Quantum General Rel-

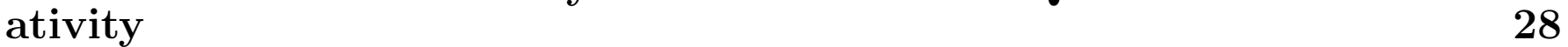

$\mathbb{\Pi I . 1}$ Mathematical Foundations $\ldots \ldots \ldots \ldots \ldots \ldots \ldots \ldots$

II.1.1 $\quad$ Polarization and Preferred Poisson Algebra $\mathcal{B}$. . . . . . . . . . . . . . . . . . . . 29

II.1.2 $\quad$ Representation Theory of $\mathcal{B}$ and Suitable Kinematical Representations . . . . . . . . . 33

III.1.2.1 Curves, Paths, Graphs and Groupoids . . . . . . . . . . . . . . . . . . . 33

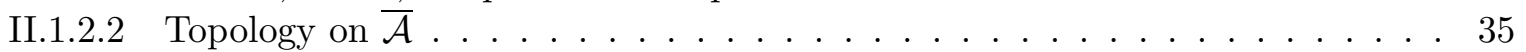

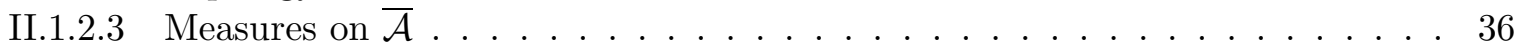

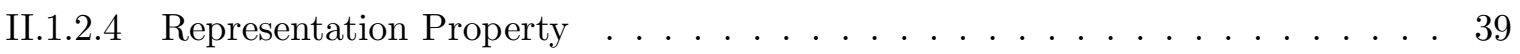

$\llbracket .2$ Quantum Kinematics . . . . . . . . . . . . . . . . . . . . . . . . 40

II.2.1 $\quad$ The Space of Solutions to the Gauss and Spatial Diffeomorphism Constraint . . . . . 41

II.2.2 $\quad$ Kinematical Geometrical Operators $\ldots \ldots \ldots \ldots \ldots$. . . . . . . . . . . . . . 42

III Selected Areas of Current Research $\quad 45$

III.1 Quantum Dynamics $\ldots \ldots \ldots \ldots \ldots \ldots \ldots \ldots \ldots$. . . . . . . . . . . . . . . 46

【II.1.1 A Possible New Mechanism for Avoiding UV Singularities in Background Independent

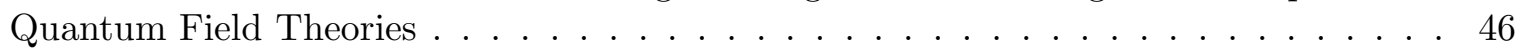

III.1.2 Sketch of a Possible Quantization of the Hamiltonian Constraint . . . . . . . . . . . 50

$\llbracket$ III.2 Loop Quantum Cosmology $\ldots \ldots \ldots \ldots \ldots \ldots \ldots \ldots$

III.2.1 A New Approach To Quantum Cosmology . . . . . . . . . . . . . . . . . . . . . . 56

III.2.2 Spectacular Results $\ldots \ldots \ldots \ldots \ldots$

III.3 Path Integral Formulation: Spin Foam Models . . . . . . . . . . . . . . . . . . . . . . 58

III.3.1 Spin Foams from the Canonical Theory $\ldots \ldots \ldots \ldots$. . . . . . . . . . . . . . 58

III.3.2 Spin Foams and BF - Theory . . . . . . . . . . . . . . . . . . . . . . . . . 59

III.4 Quantum Black Holes $\ldots \ldots \ldots$. . . . . . . . . . . . . . . . . . . . . . . . . . 62

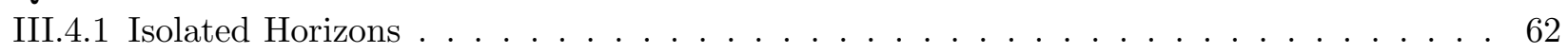

III.4.2 Entropy Counting $\ldots \ldots \ldots \ldots \ldots \ldots \ldots$ 
III.5 Semiclassical Analysis . . . . . . . . . . . . . . . . . . . . . . . . . . . 67

III.5.1 The Complexifier Machinery for Generating Coherent States . . . . . . . . . . . . . . 67

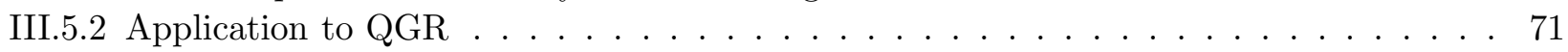

III.6 Gravitons . . . . . . . . . . . . . . . . . . . . . . . . . . . . . . . . . . . 75

III.6.1 The Isomorphism . . . . . . . . . . . . . . . . . . . . . . . . . . . . . . . 75

III.6.2 Induced Fock Representation With Polymer - Excitations . . . . . . . . . . . . . . . 77

IV Selection of Open Research Problems 80 
This is the expanded version of a talk given by the author at the 271st WE Heraeus Seminar "Aspects of Quantum Gravity: From Theory to Experimental Search", Bad Honnef, Germany, February 25th - March 1st 2002, http://www.uni-duesseldorf.de/QG-2002.

Basically, we summarize the present status of Canonical Quantum General Relativity (QGR), also known as "Loop Quantum Gravity". Our presentation tries to be precise and at the same time technically not too complicated by skipping the proofs of all the statements made. These many missing details, which are relevant to the serious reader, can be found in the notation used here in this overview e.g. in the recent, close to exhaustive review [1] and references therein. Of course, in order to be useful as a text for first reading we did not include all the relevant references here. We apologize for that to the researchers in the field but we hope that a close to complete list of their work can be found in [1]. Nice reports, treating complementary subjects of the field and more general aspects of quantum gravity can be found in [2].

The text is supplemented by numerous exercises of varying degree of difficulty whose purpose is to cut the length of the exposition and to arouse interest in further studies. Solving the problems is not at all mandatory for an undertsanding of the material, however, the exercises contain further information and thus should be looked at even on a first reading.

On the other hand, if one solves the problems then one should get a fairly good insight into the techniques that are important in QGR and in principle could serve as a preparation for a diploma thesis or a dissertation in this field. The problems sometimes involve mathematics that may be unfamiliar to students, however, this should not scare off but rather encourage the serious reader to learn the necessary mathematical background material. Here is a small list of mathematical texts, from the author's own favourites, geared at theoretical and mathematical physicists, that might be helpful:

\section{- General}

A fairly good encyclopedia is

Y. Choquet-Bruhat, C. DeWitt-Morette, "Analysis, Manifolds and Physics", North Holland, Amsterdam, 1989

- General Topology

A nice text, adopting almost no prior knowledge is

J. R. Munkres, "Toplogy: A First Course", Prentice Hall Inc., Englewood Cliffs (NJ), 1980

- Differential and Algebraic Geometry

A modern exposition of this classical material can be found in

M. Nakahara, "Geometry, Topology and Physics", Institute of Physics Publishing, Bristol, 1998

- Functional Analysis

The number one, unbeatable and close to complete exposition is

M. Reed, B. Simon, "Methods of Modern Mathematical Physics", vol. 1 - 4, Academic Press, New York, 1978

especially volumes one and two.

- Measure Theory

An elementary introduction to measure theory can be found in the beautiful book

W. Rudin, "Real and Complex Analysis", McGraw-Hill, New York, 1987 
- Operator Algebras

Although we do not really make use of $C^{*}$-algebras in this review, we hint at the importance of the subject, so let us include

O. Bratteli, D. W. Robinson, "Operator Algebras and Quantum Statistical Mechanics", vol. 1,2, Springer Verlag, Berlin, 1997

- Harmonic Analysis on Groups

Although a bit old, it still contains a nice collection of the material around the Peter \& Weyl theorem:

N. J. Vilenkin, "Special Functions and the Theory of Group Representations", American Mathematical Society, Providence, Rhode Island, 1968

- Mathematical General Relativity

The two leading texts on this subject are

R. M. Wald, "General Relativity", The University of Chicago Press, Chicago, 1989

S. Hawking, Ellis, "The Large Scale Structure of Spacetime", Cambridge University Press, Cambridge, 1989

- Mathematical and Physical Foundations of Ordinary QFT

The most popular books on axiomatic, algebraic and constructive quantum field theory are

R. F. Streater, A. S. Wightman, "PCT, Spin and Statistics, and all that", Benjamin, New York, 1964

R. Haag, "Local Quantum Physics", 2nd ed., Springer Verlag, Berlin, 1996

J. Glimm, A. Jaffe, "Quantum Physics", Springer-Verlag, New York, 1987

In the first part we motivate the particular approach to a quantum theory of gravity, called (Canonical) Quantum General Relativity, and develop the classical foundations of the theory as well as the goals of the quantization programme.

In the second part we list the solid results that have been obtained so far within QGR. Thus, we will apply step by step the quantization programme outlined at the end of section I.3 to the classical theory that we defined in section 1.2. Up to now, these steps have been completed approximately until step vii) at least with respect to the Gauss - and the spatial diffeomorphism constraint. The analysis of the Hamiltonian constraint has also reached level vii) already, however, its classical limit is presently under little control which is why we discuss it in part three where current research topics are listed.

In the third part we discuss a selected number of active research areas. The topics that we will describe already have produced a large number of promising results, however, the analysis is in most cases not even close to being complete and therefore the results are less robust than those that we have obtained in the previous part.

Finally, in the fourth part we summarize and list the most important open problems that we faced during the discussion in this report. 


\section{Part I}

\section{Motivation and Introduction}




\section{I.1 Motivation}

\section{I.1.1 Why Quantum Gravity in the 21'stCentury ?}

Students that plan to get involved in quantum gravity research should be aware of the fact that in our days, when financial resources for fundamental research are more and more cut and/or more and more absorbed by research that leads to practical apllications on short time scales, one should have a good justification for why tax payers should support any quantum gravity research at all. This seems to be difficult at first due to the fact that even at CERN's LHC we will be able to reach energies of at most $10^{4} \mathrm{GeV}$ which is fifteen orders of magnitude below the Planck scale which is the energy scale at which quantum gravity is believed to become important. Therefore one could argue that quantum gravity research in the 21 'st century is of purely academic interest only.

To be sure, it is a shame that one has to justify fundamental research at all, a situation unheard of in the beginning of the 20'th century which probably was part of the reason for why so many breakthroughs especially in fundamental physics have happened in that time. Fundamental research can work only in absence of any pressure to produce (mainstream) results, otherwise new, radical and independent thoughts are no longer produced. To see the time scale on which fundamental research leads to practical results, one has to be aware that General Relativity (GR) and Quantum Theory (QT) were discovered in the 20's and 30's already but it took some 70 years before quantum mechanics through, e.g. computers, mobile phones, the internet, electronic devices or general relativity through e.g. space travel or the global positioning system (GPS) became an integral part of life of a large fraction of the human population. Where would we be today if the independent thinkers of those times were forced to do practical physics due to lack of funding for analyzing their fundamental questions?

Of course, in the beginning of the 20'th century, one could say that physics had come to some sort of crisis, so that there was urgent need for some revision of the fundamental concepts: Classical Newtonian mechanics, classical electrodynamics and thermodynamics were so well understood that Max Planck himself was advised not to study physics but engineering. However, although from a practical point of view all seemed well, there were subtle inconsistencies among these theories if one drove them to their logical frontiers. We mention only three of them:

1) Although the existence of atoms was by far not widely accepted at the end of the 19th century (even Max Planck denied them), if they existed then there was a serious flaw, namely, how should atoms be stable ? Acceralated charges radiate Bremsstrahlung according to Maxwell's theory, thus an electron should fall into the nucleus after a finite amount of time.

2) If Newton's theory of absolute space and time was correct then the speed of light should depend on the speed of the inertial observer. The fact that such velocity dependence was ruled out to quadratic order in $v / c$ in the famous Michelson-Morley experiment was explained by postulating an unknown medium, called ether, with increasingly (as measurement precision was refined) bizarre properties in order to conspire to a negative outcome of the interferometer experiment and to preserve Newton's notion of space and time.

3) The precession of mercury around the sun contradicted the ellipses that were predicted by Newton's theory of gravitation.

Today we easily resolve these problems by 1) quantum mechanics, 2) special relativity and 3) general relativity. Quantum mechanics does not allow for continuous radiation but predicts a discrete energy spectrum of the atom, special relativity removed the absolute notion of space and time and general relativity generalizes the static Minkowski metric underlying special relativity to a dynamical theory of a metric field which revolutionizes our understanding of gravity not as a force but as 
geometry. Geometry is curved at each point in a manifold proportional to the matter density at that point and in turn curvature tells matter what are the straightest lines (geodesics) along which to move. The ether became completely unnecessary by changing the foundation of physics and beautifully demonstrates that driving a theory to its logical frontiers can make extra structures redundant, what one had to change is the basic principles of physics. $\square$

This historic digression brings us back to the motivation for studying quantum gravity in the beginning of the 21st century. The question is whether fundamental physics also today is in a kind of crisis. We will argue that indeed we are in a situation not unsimilar to that of the beginning of the 20th century:

Today we have very successful theories of all interactions. Gravitation is described by general relativity, matter interactions by the standard model of elementary particle physics. As classical theories, their dynamics is summarized in the classical Einstein equations. However, there are several problems with these theories, some of which we list below:

i) Classical - Quantum Inconsistency

The fundamental principles collide in the classical Einstein equations

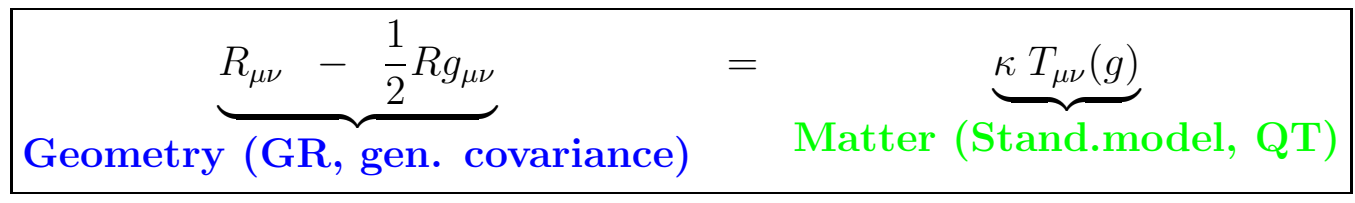

These equations relate matter density in form of the energy momentum tensor $T_{\mu \nu}$ and geometry in form of the Ricci curvature tensor $R_{\mu \nu}$. Notice that the metric tensor $g_{\mu \nu}$ enters also the definition of the energy momentum tensor. However, while the left hand side is described until today only by a classical theory, the right hand side is governed by a quantum field theory (QFT). Since complex valued functions and operators on a Hilbert space are two completely different mathematical objects, the only way to make sense out of the above equations while keeping the classical and quantum nature of geometry and matter respectively is to take expectation values of the right hand side, that is,

$$
R_{\mu \nu}-\frac{1}{2} R g_{\mu \nu}=\kappa<\hat{T}_{\mu \nu}\left(g_{0}\right)>, \quad \kappa=8 \pi G_{\text {Newton }} / c^{3}
$$

Here $g_{0}$ is an arbitrary background metric, say the Minkowski metric $\eta=\operatorname{diag}(-1,1,1,1)$. However, even if the state with respect to which the expectation value is taken is the vacuum state $\psi_{g_{0}}$ with respect to $g_{0}$ (the notion of vacuum depends on the background metric itself, see below), the right hand side is generically non-vanishing due to the vacuum fluctuations, enforcing $g=g_{1} \neq g_{0}$. Hence, in order to make this system of equations consistent, one could iterate the procedure by computing the vacuum state $\psi_{g_{1}}$ and reinserting $g_{1}$ into $\hat{T}_{\mu \nu}($.$) ,$ resulting in $g_{2} \neq g_{1}$ etc. hoping that the procedure converges. However, this is generically not the case and results in "run - away solutions" [3].

Hence, we are enforced to quantize the metric itself, that is, we need a quantum theory of

${ }^{1}$ Notice, that the stability of atoms is still not satisfactorily understood even today because the full problem also treats the radiation field, the nucleus and the electron as quantum objects which ultimately results in a problem in QED, QFD and QCD for which we have no entirely satisfactory description today, see below. 
gravity resulting in the

$$
\begin{gathered}
\widehat{\boldsymbol{R}}_{\mu \nu}-\frac{1}{2} \widehat{\boldsymbol{R}} \widehat{\boldsymbol{g}}_{\mu \nu}=\kappa \widehat{T}_{\mu \nu}(\widehat{\boldsymbol{g}}) \\
\text { Quantum - Einstein - Equations }
\end{gathered}
$$

The inverted commas in this equation are to indicate that this equation is to be made rigorous in a Hilbert space context. QGR is designed to exactly do that, see section [II.1.

ii) General Relativity Inconsistencies

It is well-known that classical general relativity is an incomplete theory because it predicts the existence of so-called spacetime singularities, regions in spacetime where the curvature or equivalently the matter density becomes infinite [4]. The most prominent singularities of this kind are black hole and big bang singularities and such singularities are generic as shown in the singularity theorems due to Hawking and Penrose. When a singularity appears it means that the theory has been pushed beyond its domain of validity, certainly when matter collapses it reaches a state of extreme energy density at which quantum effects become important. A quantum theory of gravity could be able to avoid these singularities in a similar way as quantum mechanics explains the stability of atoms. We will see that QGR is able to achieve this, at least in the simplified context of "Loop Quantum Cosmology", see section III.2.

iii) Quantum Field Theory Inconsistencies

As is well-known, QFT is plagued by UV (or short distance) divergences. The fundamental operators of the theory are actually not operators but rather operator valued distributions and usually interesting objects of the theory are (integrals of) polynomials of those evaluated in the same point. However, the product of distributions is, by definition, ill-defined. The appearance of these divergences is therefore, on the one hand, not surprising, on the other hand it indicates again that the theory is incomplete: In a complete theory there is no room for infinities. Thus, either the appropriate mathematical framework has not been found yet, or they arise because one neglected the interaction with the gravitational field. In fact, in renormalizable theories one can deal with these infinities by renormalization, that is, one introduces a short distance cut-off (e.g. by point splitting the operator valued distributions) and then redefines masses and coupling constants of the theory in a cut-off dependent way such that they stay finite as the cut-off is sent to zero. This redefinition is done in the framework of perturbation theory (Feynman diagrammes) by subtracting counter terms from the original Lagrangean which are formally infinite and a theory is said to be renormalizable if the number of algebraically different such counter terms is finite.

The occurance of UV singularities is in deep conflict with general relativity due to the following reason: In perturbation theory, the divergences have their origin in Feynman loop integrals in momentum space where the inner loop 4-momentum $k=(E, P)$ can become arbitrarily large, see figure 1 for an example from QED (mass renormalization). Now such virtual (offshell) particles with energy $E$ and momentum $P$ have a spatial extension of the order of the Compton radius $\lambda=\hbar / P$ and a mass of the order of $E / c^{2}$. Classical general relativity predicts that this lump of energy turns into a black hole once $\lambda$ reaches the Schwarzschild radius of 


$$
\mathrm{p}-\mathrm{k}
$$

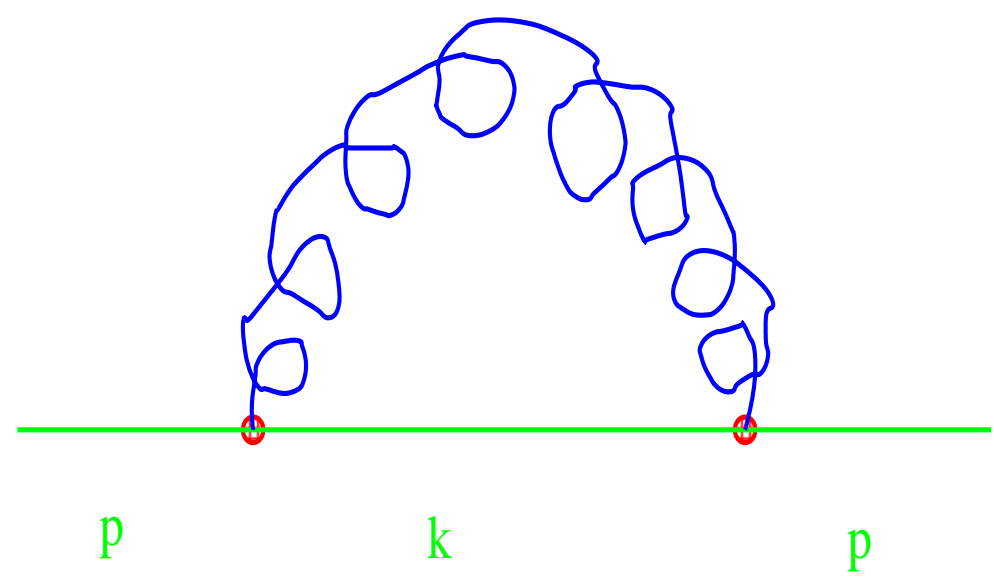

Figure 1: One loop correction to the electron propagator in QED

the order of $r=G E / c^{4}$. In a Lorentz frame where $E \approx P c$ this occurs at the Planck energy $E=E_{P}=\sqrt{\hbar / \kappa} c \approx 10^{19} \mathrm{GeV}$ or at the Planck length Compton radius $\ell_{P}=\sqrt{\hbar \kappa} \approx 10^{-33} \mathrm{~cm}$. However, when a (virtual) particle turns into a black hole it completely changes its properties. For instance, if the virtual particle is an electron then it is able to interact only electroweakly and thus can radiate only particles of the electrowak theory. However, once a black hole has formed, also Hawking processes are possible and now any kind of particles can be emitted, but at a different production rate. Of course, this is again an energy regime at which quantum gravity must be important and these qualitative pictures must be fundamnetally wrong, however, they show that there is a problem with integrating virtual loops into the UV regime. In fact, these qualitative thoughts suggest that gravity could serve as a natural cut-off because a black hole of Planck mass size $\ell_{P}$ should decay within a Planck time unit $t_{P}=\ell_{P} / c \approx 10^{-43} s$ so that one has to integrate $P$ only until $E_{P} / c$. Moreover, it indicates that spacetime geometry itself acquires possibly a discrete structure since arguments of this kind make it plausible that it is impossible to resolve spacetime distances smaller than $\ell_{P}$, basically because the spacetime behind an event horizon is in some sense "invisible". These are, of course, only hopes and must be demonstrated within a concrete theory. We will see that QGR is able to precisely do that and its fundamental discreteness is in particular responsible for why the Bekenstein Hawking

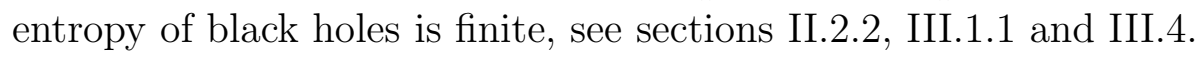

So we see that there is indeed a fundamental inconsisteny within the current description of fundamental physics comparable to the time before the discovery of GR and QT and its resolution, Quantum Gravity, will revolutionize not only our understanding of nature but will also drive new kinds of technology that we do not even dare to dream of today.

\section{I.1.2 The Role of Background Independence}

Given the fact that both QT and GR were discovered already more than 70 years ago and that people have certainly thought about quantizing GR since then and that matter interactions are more or less succesfully described by ordinary quantum field theories (QFT), it is somewhat surprising that we do not yet have a quantum gravity theory. Why is it so much harder to combine gravity with the principles of quantum mechanics than for the other interactions? The short answer is that 


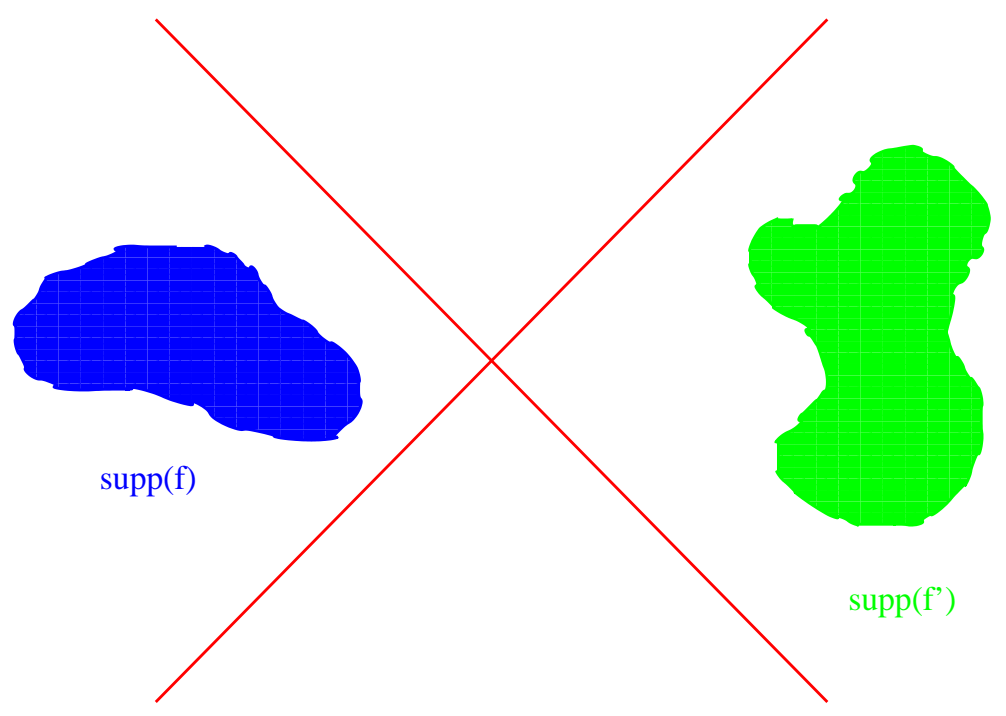

Figure 2: Spacelike separated regions in Minkowski space

\section{Ordinary QFT only incorporates Special Relativity.}

To see why, we just have to remember that ordinary QFT has an axiomatic definition, here for a scalar field for simplicity:

WIGHTMAN AXIOMS (Scalar Fields on Minkowsk Space)

W1 Poincaré Group P:

$\exists$ continuous, unitary representation $\hat{U}$ of $\mathcal{P}$ on a Hilbert space $\mathcal{H}$.

W2 Forward Lightcone Spektral Condition:

For the generators $\hat{P}^{\mu}$ of the translation subgroup of $\mathcal{P}$ holds $\eta_{\mu \nu} \hat{P}^{\mu} \hat{P}^{\nu} \leq 0, \quad \hat{P}^{0} \geq 0$.

W3 Existence and Uniqueness of a $\mathcal{P}$-inariant Vacuum $\Omega$ :

$\hat{U}(p) \Omega=\Omega \forall p \in \mathcal{P}$.

W4 P-Covariance:

$$
\begin{gathered}
\hat{\phi}(f):=\int d^{D+1} x f(x) \hat{\phi}(x), \quad f \in \mathcal{S}\left(R^{D+1}\right) \\
\hat{\phi}\left(f_{1}\right) . . \hat{\phi}\left(f_{n}\right) \Omega \text { dense in } \mathcal{H} \text { and } \hat{U}(p) \hat{\phi}(f) \hat{U}(p)^{-1}=\hat{\phi}(f \circ p)
\end{gathered}
$$

W5 Locality (Causality):

If $\operatorname{supp}(f), \operatorname{supp}\left(f^{\prime}\right)$ spacelike separated (see figure 2), then $\left[\hat{\phi}(f), \hat{\phi}\left(f^{\prime}\right)\right]=0$.

It is obvious that due to the presence of the Minkowski backgrounde metric $\eta$ we have available a large amount of structure which forms the fundament on which ordinary QFT is built. Roughly, we 
have the following scheme:

$$
\begin{aligned}
& \underset{\text { b.-metric }}{\eta_{\mu \nu}} \Rightarrow \begin{array}{c}
\mathcal{P} \\
\text { symm.-group }
\end{array} \Rightarrow \begin{array}{c}
\hat{H}=\hat{P}^{0} \\
\text { Ham.-operator }
\end{array} \\
& \Downarrow
\end{aligned}
$$

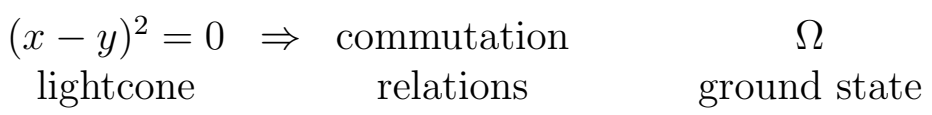

Notice that a generic background metric has no symmetry group at all so that it is not straightforward to generalize these axioms to QFT on general curved backgrounds, however, since any metric is locally diffeomorphic to the Minkowski metric, a local generalization is possible and results in the so-called microlocal analysis in which the role of vacuum states is played by Hadamard states, see e.g. [5].

\section{The fundamental, radically new feature of Einstein's theory is that there is no background metric at all: The theory is background independent. The lightcones themselves are fluctuating, causality and locality become empty notions. The dome of ordinary QFT completely collapses.}

Of course, there must be a regime in any quantum gravity theory where the quantum fluctuations of the metric operator are so tiny that we recover the well established theory of free ordinary quantum fields on a given background metric, however, the large fluctuations of the metric operator can no longer be ignored in extreme astrophysical or cosmological situations, such as near a black hole or big bang singularity.

People have tried to rescue the framework of ordinary QFT by splitting the metric into a background piece and a fluctuation piece

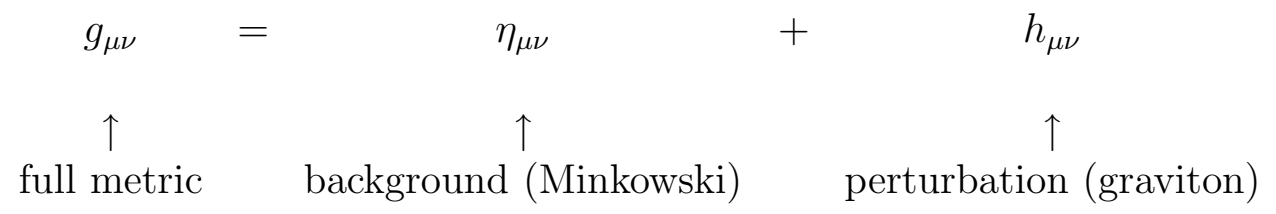

which results in a Lagrangean for the graviton field $h_{\mu \nu}$ and could in principle be the definition of a graviton QFT on Minkowski space. However, there are serious drawbacks:

i) Non-Renormalizability

The resulting theory is perturbatively non-renormalizable [6] as could have been expected from the fact that the coupling constant of the theory, the Planck area $\ell_{P}^{2}$, has negative mass dimension (in Planck units). Even the supersymmetric extension of the theory, in any possible dimension has this bad feature [7]. It could be that the theory is non-perturbatively renormalizable, meaning that it has a non-Gaussian fix point in the language of Wilson, a possibility that has recently regained interest [8].

ii) Violation of Background Independence

The split of the metric performed above again distinguishes the Minkowski metric among all others and reintroduces therefore a background dependence. This violates the key feature of Einstein's theory and thus somehow does not sound correct, we better keep background 
independence if we want to understand how quantum mechanics can possibly work together with general covariance.

\section{iii) Violation of Diffeomorphism Covariance}

The split of the metric performed above is certainly not diffeomorphism covariant, it breaks the diffeomorphism group down to Poincaré group. Violation of fundamental, local gauge symmetries is usually considered as a bad feature in Yang-Mills theories on which all the other interactions are based, thus already from this point of view perturbation theory looks dangerous. As a side remark we see that background dependence and violation of general covariance are synonymous.

\section{iv) Gravitons and Geometry}

Somehow the whole idea of the gravitational interaction as a result of graviton exchange on a background metric contradicts Einstein's original and fundamental idea that gravity is geometry and not a force in the usual sense. Therefore such a perturbative description of the theory is very unnatural from the outset and can have at most a semi-classical meaning when the metric fluctuations are very tiny.

\section{v) Gravitons and Dynamics}

All that classical general relativity is about is how a metric evolves in time in an interplay with the matter present. It is clear that an initially (almost) Minkowskian metric can evolve to something that is far from Minkowskian at other times, an example being cosmological big bang situations or the collapse of initially dilluted matter (evolved backwards). In such situations the assumption being made in (I.1.2.1), namely that $h$ is "small" as compared to $\eta$ is just not dynamically stable. In some sense it is like trying to use Cartesian coordinates for a sphere which can work at most locally.

All these points just naturally ask for a non-perturbative approach to quantum gravity. This, in turn, could also cure another unpleasant feature about ordinary QFT: Today we do not have a single example of a rigorously defined interacting ordinary QFT in four dimensions, in other words, the renormalizable theories that we have are only defined order by order in perturbation theory but the perturbation series diverges. A non-perturbative definition, to which we seem to be forced when coupling gravity anyway, might change this unsatisfactory situation.

It should be noted here that there is in fact a consistent perturbative description of a candidate quantum gravity theory, called string theory (or $\mathrm{M}$ - Theory nowadays) 9. 9 However, in order to achieve this celebrated rather non-trivial result, expectedly one must introduce extra structure: The theory lives in 10 (or 11) rather than 4 dimensions, it is necessarily supersymmetric and it has an infinite number of extra particles besides those that are needed to make the theory compatible with the standard model. Moreover, at least as presently understood, again the fundamental new ingredient of Einstein's theory, background independence, is violated in string theory. This current background dependence of string theory is supposed to be overcome once M - Theory has been rigorously defined.

At present only string theory has a chance to explain the matter content of our universe. The unification of symmetries is a strong guiding principle in physics as well and has been pushed also

\footnotetext{
${ }^{2}$ String theory is an ordinary QFT but not in the usual sense: It is an ordinary scalar QFT on a 2d Minkowski space, however, the scalar fields themselves are coordinates of the ambient target Minkowski space which in this case is 10 dimensional. Thus, it is similar to a first quantized theory of point particles. The theory is renormalizable and presumably even finite order by order in perturbation theory but the perturbation series does not converge.
} 


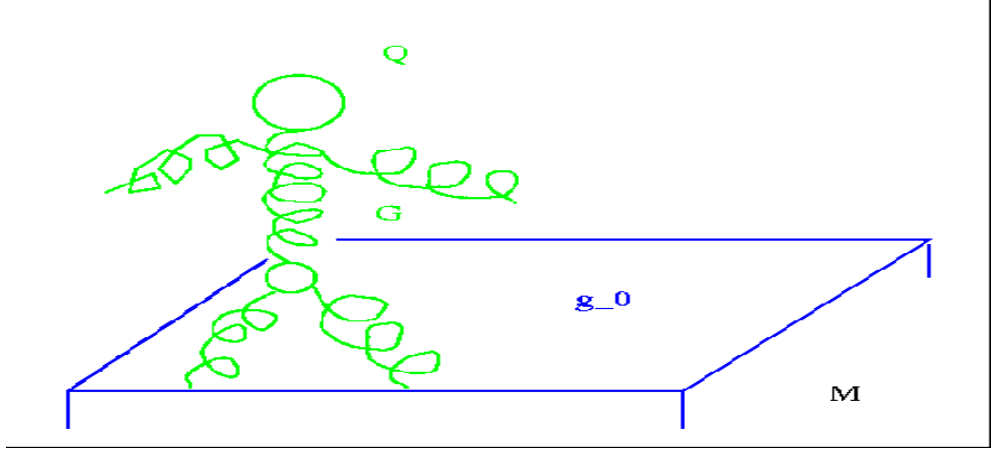

Figure 3: QFT on Background Spacetime $\left(M, g_{0}\right)$ : Actor: Matter, Stage: Geometry + Manifold $M$.

by Einstein in his programme of geometrization of physics attemting to unify electromagnetism and gravity in a five-dimensional Kaluza - Klein theory. The unification of the electromagnetic and the weak force in the electroweak theory is a prime example for the success of such ideas. However, unification of forces is an additional principle completely independent of background independence and is not necessarily what a quantum theory of gravity must achieve: Unification of forces can be analyzed at the purely classical leve阳. Thus, the only question is whether the theory can be quantized before unification or not (should unification of geometry and matter be realized in nature at all).

We are therefore again in a situation, similar to that before the discovery of special relativity, where we have the choice between a) preserving an old principle, here renormalizability of perturbative QFT on background spacetimes $(M, \eta)$, at the price of introducing extra structure (extra unification symmetry), or b) replacing the old principle by a new principle, here non-perturbative QFT on a differentiable manifold $M$, without new hypothetical structure. At this point it unclear which methodology has more chances for success, historically there is evidence for either of them (e.g. the unification of electromagnetism and the massive Fermi model is evidence for the former, the replacement of Newton's notion of spactime by special relativity is evidence for the latter) and it is quite possible that we actually need both ideas. In QGR we take the latter point of view to begin with since there maybe zillions of ways to unify forces and it is hard to judge whether there is a "natural one", therefore the approach is purposely conservative because we actually may be able to derive a natural way of unification, if necessary, if we drive the theory to its logical frontiers. Among the various non-perturbative approaches available we will choose the canonical one.

Pictorially, one could illustrate the deep difference between a background dependent QFT and background independent QFT as follows: In figure 3 we see matter in the form of QCD (notice the quark $(Q)$ propagators, the quark-gluon vertices and the three - and four point gluon $(G)$ vertices) displayed as an actor in green. Matter propagates on a fixed background spacetime $g_{0}$ according to well-defined rules, particles know exactly what timelike geodesics are etc. This fixed background

\footnotetext{
${ }^{3}$ In fact, e.g. the unified electroweak $S U(2)_{L} \times U(1)$ theory with its massless gauge bosons can be perfectly described by a classical Lagrangean. The symmetry broken, massive $U(1)$ theory can be derived from it, also classically, by introducing a constant background Higgs field (Higgs mechanism) and expanding the symmetric Lagrangean around it. It is true that the search for a massless, symmetric theory was inspired by the fact that a theory with massive gauge bosons is not renormalizable (so the motivation comes from quantum theory) and, given the non-renormalizability of general relativity, many take this as an indication that one must unify gravity with matter, one incarnation of which is string theory. However, the argument obviously fails should it be possible to quantize gravity non-perturbatively.
} 


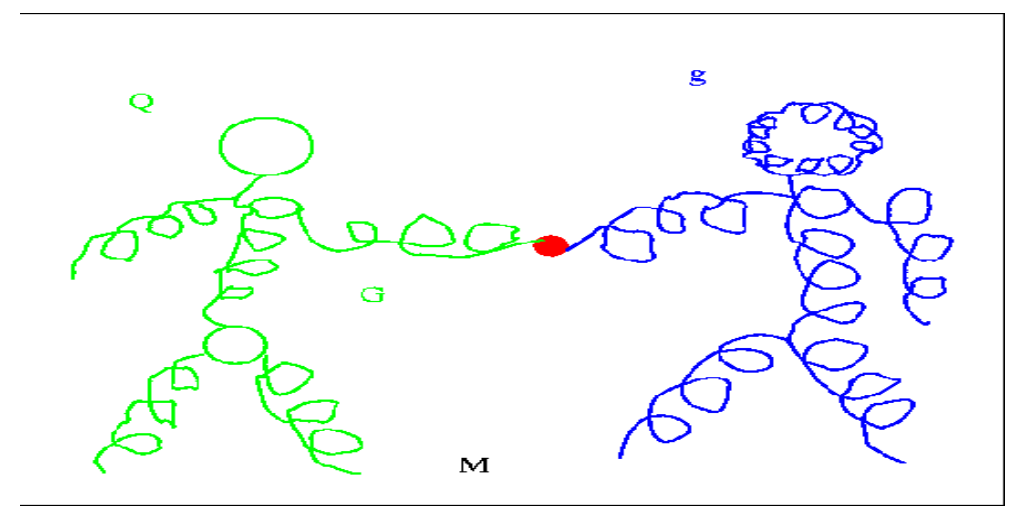

Figure 4: QGR on Differential Manifold $M$ : Actor: Matter + Geometry, Stage: Manifold $M$

spacetime $g_{0}$ is displayed as a firm stage in blue. This is the situation of a QFT on a Background Spacetime.

In contrast, in figure the stage has evaporated, it has become itself an actor (notice the arbitrarily high valent graviton $(g)$ vertices) displayed in blue as well. Both matter and geometry are now dynamical entities and interact as displayed by the red vertex. There are no light cones any longer, rather the causal structure is a semiclassical concept only. This is the situation of a QFT on a Differential Manifold and this is precisely what QGR aims to rigorously define.

It is clear from these figures that the passage from a QFT on a background spacetime to a QFT on a differential manifold is a very radical one: It is like removing the chair on which you sit and trying to find a new, yet unknown, mechanism that keeps you from falling down. We should mention here that for many researchers in quantum gravity even that picture is not yet radical enough, some proposals require not only to get rid of the background metric $g_{0}$ but also of the differential manifold, allowing for topology change. This is also very desired in QGR but considered as a second step. In 3d QGR also this step could be completed and the final picture is completely combinatorial.

Let us finish this section by stating once more what we mean by Quantum General Relativity (QGR). 


\section{DEFINITION}

(Canonical) Quantum General Relativity (QGR) is an attempt to construct a mathematically rigorous, non - perturbative, background independent Quantum Field Theory of four - dimensional, Lorentzian General Relativity and all known matter in the continuum.

No additional, experimentally unverified structures are introduced. The fundamental principles of General Covariance and Quantum Theory are brought together and driven to their logical frontiers guided by mathematical consistency.

QGR is not a unified theory of all interactions in the standard sense since unification of gauge symmetry groups is not necessarily required in a non-perturbative approach. However, Geometry and Matter are unified in a non - standard sense by making them both transform covariantly under the Diffeomorphism Group at the quantum level.

\section{I.2 Introduction: Classical Canonical Formulation of Gen- eral Relativity}

In this section we sketch the classical Hamiltonian formulation of general relativity in terms of Ashtekar's new variables. There are many ways to arrive at this new formulation and we will choose the one that is the most convenient one for our purposes.

The Hamiltonian formulation by definition requires some kind of split ot the spacetime variables into time and spatial variables. This seems to contradict the whole idea of general covariance, however, quantum meachanics as presently formulated requires a notion of time because we interpret expection values of operators as instantaneous measurement values averaged over a large number of measurements. In order to avoid this one has to "covariantize" the interpretation of quantum mechanics, in particular the measurement process, see e.g. [10] for a discussion. There are a number of proposals to make the canonical formulation more covariant, e.g.t: Multisymplectic Ansätze [13] in which there are multimomenta, one for each spacetime dimension, rather than just one for the time coordinate; Covariant phase space formulations [14] where one works on the space of solutions to the field equations rather than on the intial value instantaneous phase space; Peierl's bracket formulations [15] which covariantize the notion of the usual Poisson bracket; history bracket formulations [16], which grew out of the consistent history formulation of quantum mechanics [17], and which extends

\footnotetext{
${ }^{4}$ Path integrals [1] use the Lagrangean rather than the Hamiltonian and therefore seem to be better suited to a covariant formulation than the canonical one, however, usually the path integral is interpreted as some sort of propagator which makes use of instantaneous time Hilbert spaces again which therefore cannot be completely discarded with. At present, this connection with the canonical formulation is not very transparent, part of the reason being that the path integral is usually only defined in its Euclidean formulation, however the very notion of analytic continuation in time is not very meaningful in a theory where there is no distinguished choice of time, see however [12] for recent progress in this direction.
} 
the usual spatial Poisson bracket to spacetime.

At the classical level all these formulations are equivalent. However, at the quantum level, one presently gets farthest within the the standard canonical formulation: The quantization of the multisymplectic approach is still in its beginning, see [18] for the most advanced results in this respect; The covariant phase space formulation is not only very implicit because one usually does not know the space of solutions to the classical field equations, but even if one manages to base a quantum theory on it, it will be too close to the classical theory since certainly the singularities of the classical theory are also built into the quantum theory; The Peierl's bracket also needs the explicit space of solutions to the classical field equations; Also the quantization of the history bracket formulation just has started, see [19] for first steps in that direction.

Given this present status of affairs, we will therefore proceed with the standard canonical quantization and see how far we get. Notice that there is no obvious problem with general covariance: For instance, standard Maxwell theory can be quantized canonically without any problem and one can show that the theory is Lorentz covariant although the spacetime split into space and time seems to break the Lorentz group down to the rotation group. This is not at all the case! It is just that Lorentz covariance is not manifest, one has to do some work in order to establish Lorentz covariance. Indeed, as we will see, at least at the classical level we will explicitly recover the four-dimensional diffeomorphism group in the formalism, although it is admittedly deeply hiddeen in the canonical formalism.

With these cautionary remarks out of the way, we will thus assume that the four dimensional spacetime manifold has the topology $\mathbb{R} \times \sigma$, where $\sigma$ is a three dimensional manifold of arbitrary topology, in order to perform the $3+1$ split. This assumption about the topology of $M$ may seem rather restrictive, however, it is not due to the following reasons: (1) According to a theorem due to Geroch any globally hyperbolic manifold (roughly those that admit a smooth metric with everywhere Lorentzian signature) are necessarily of that topology. Since Lorentzian metrics are what we are interested in, at least classically the assumption about the topology of $M$ is forced on us. (2) Any four manifold $M$ has the topology of a countable disjoint union $\cup_{\alpha} I_{\alpha} \times \sigma_{\alpha}$ where either $I_{\alpha}$ are open intervals and $\sigma_{\alpha}$ is a three manifold or $I_{\alpha}$ is a one point set and $\sigma_{\alpha}$ is a two manifold (the latter are the intersections of the closures of the former). In this most generic situation we thus allow topology change between different three manifolds and it is even classically an open question how to make this compatible with the action principle. We take here a practical point of view and try to understand the quantum theory first for a single copy of the form $\mathbb{R} \times \sigma$ and later on worry how we glue the theories for different $\sigma^{\prime} s$ together.

\section{I.2.1 The ADM Formulation}

In this nice situation the $3+1$ split is well known as the Arnowitt - Deser - Misner (ADM) formulation of general relativity, see e.g. [四] and we briefly sketch how this works. Since $M$ is diffeomorphic to $\mathbb{R} \times \sigma$ we know that $M$ foliates into hypersurfaces $\Sigma_{t}, t \in \mathbb{R}$ as in figure 5, where $t$ labels the hypersurface and will play the role of our time coordinate. If we denote the four dimensional coordinates by $X^{\mu}, \mu=0,1,2,3$ and the three dimensional coordinates by $x^{a}, a=1,2,3$ then we know that there is a diffeomorphism $\varphi: \mathbb{R} \times \sigma \rightarrow M ;(t, x) \mapsto X=\varphi(t, x)$ where $\Sigma_{t}=\varphi(t, \sigma)$. We stress that the four diffeomorphism $\varphi$ is completely arbitrary until this point and thus the foliation of $M$ is not at all fixed, in other words, the set of foliations is in one to one correspondence with $\operatorname{Diff}(M)$, the four dimensional diffeomorphism group. Consider the tangential vector fields to $\Sigma_{t}$ given by

$$
S_{a}(X):=\left(\partial_{a}\right)_{\varphi(t, x)=X}=\left(\varphi_{, a}^{\mu}(t, x)\right)_{\varphi(t, x)=X} \quad \partial_{\mu}
$$




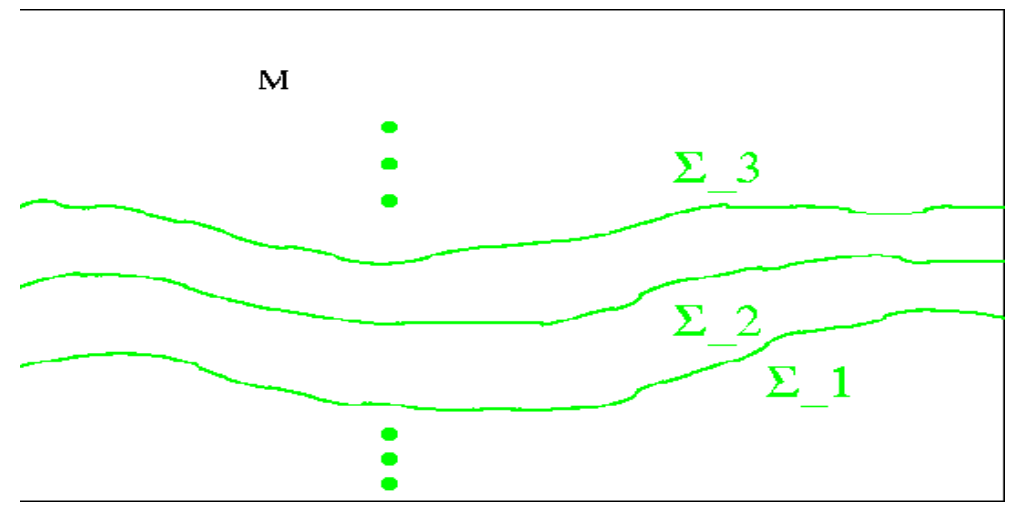

Figure 5: Foliation of $M$

Denoting the four metric by $g_{\mu \nu}$ we define a normal vector field $n^{\mu}(X)$ by $g_{\mu \nu} n^{\mu} S_{a}^{\nu}=0, g_{\mu \nu} n^{\mu} n^{\nu}=-1$. Thus, while the tangential vector fields depend only on the foliation, the normal vector field depends also on the metric. Let us introduce the foliation vector field

$$
T(X):=\left(\partial_{t}\right)_{\varphi(t, x)=X}=\left(\varphi_{, t}^{\mu}(t, x)\right)_{\varphi(t, x)=X} \partial_{\mu}
$$

and let us decompose it into the basis $n, S_{a}$. This results in

$$
T=N n+U^{a} S_{a}
$$

where $N$ is called the lapse function while $U^{a} S_{a}$ is called the shift vector field. The arbitrariness of the foliation is expressed in the arbitrariness of the fields $N, U^{a}$. We can now introduce two symmetric spacetime tensor fields ( $\nabla$ is the unique, torsion free covariant differential compatible with $g_{\mu \nu}$ )

$$
q_{\mu \nu}=g_{\mu \nu}+n_{\mu} n_{\nu}, \quad K_{\mu \nu}=q_{\mu \rho} q_{\nu \sigma} \nabla^{\rho} n^{\nu}
$$

called the intrinsic metric and the extrinsic curvature respectively which are spatial, that is, their contraction with $n$ vanishes. Thus, their full information is contained in their components with respect to the spatial fields $S_{a}$, e.g. $q_{a b}(t, x)=\left[q_{\mu \nu} S_{a}^{\mu} S_{b}^{\nu}\right](X(t, x))$. In particular,

$$
K_{a b}(t, x)=\frac{1}{2 N}\left[\dot{q}_{a b}-\mathcal{L}_{U} q_{a b}\right]
$$

contains information about the velocity of $q_{a b}$. Here $\mathcal{L}$ the Lie derivative. The metric $g_{\mu \nu}$ is completely specified in terms of $q_{a b}, N, U^{a}$ as one easily sees by expressing the line element $d s^{2}=g_{\mu \nu} d X^{\mu} d X^{\nu}$ in terms of $d t, d x^{a}$.

\section{Exercise I.2.1.}

Recall the definition of the Lie derivative and verify that $K_{\mu \nu}$ is indeed symmetric and that formula (1.2.1.5) holds.

\section{Hint:}

A hypersurface $\Sigma_{t}$ can be defined by the solution of an equation of the form $\tau(X)=t$. Conclude that $n_{\mu} \propto \nabla_{\mu} \tau$ and use torsion - freness of $\nabla$.

The Legendre transformation of the Einstein-Hilbert action

$$
S=\frac{1}{\kappa} \int_{M} d^{4} X \sqrt{|\operatorname{det}(g)|} R^{(4)}
$$


with $q_{a b}, N, U^{a}$ considered as configuration coordinates in an infinite dimensional phase space is standard and we will not repeat the analysis here, which uses the so - called Gauss - Codacci equations.

Here we are considering for simplicity only the case that $\sigma$ is compact, otherwise (I.2.1.6) would contain boundary terms. The end result is

$$
S=\frac{1}{\kappa} \int_{\mathbb{R}} d t \int_{\sigma} d^{3} x\left\{\dot{q}_{a b} P^{a b}+\dot{N} P+\dot{N}^{a} P_{a}-\left[\lambda P+\lambda^{a} P_{a}+U^{a} V_{a}+N C\right]\right\}
$$

where

$$
P^{a b}=\frac{\kappa \delta S}{\delta q_{a b}}=\sqrt{\operatorname{det}(q)}\left[q^{a c} q^{b d}-q^{a b} q^{c d}\right] K_{c d}
$$

and $P, P_{a}$ are the momentua conjugate to $q_{a b}, N, U^{a}$ respectively. Thus, we have for instance the equal time Poisson brackets

$$
\left\{P^{a b}(t, x), P^{c d}(t, y)\right\}=\left\{q_{a b}(t, x), q_{c d}(t, y)\right\}=0, \quad\left\{P^{a b}(t, x), q_{c d}(t, y)\right\}=\kappa \delta_{(c}^{a} \delta_{d)}^{b} \delta(x, y)
$$

where $(.)_{(a b)}:=\left[(.)_{a b}+(.)_{b a}\right] / 2$ denotes symmetrization. The functions $C, V_{a}$ which depend only on $q_{a b}, P^{a b}$ are called the Hamiltonian and Spatial Diffeomorphism constraint respectively for reasons that will become obvious in a moment. Their explicit form is given by

$$
\begin{aligned}
V_{a} & =-2 q_{a c} D_{b} P^{b c} \\
C & =\frac{1}{\sqrt{\operatorname{det}(q)}}\left[q_{a c} q_{b d}-\frac{1}{2} q_{a b} q_{c d}\right] P^{a b} P^{c d}-\sqrt{\operatorname{det}(q)} R
\end{aligned}
$$

where $D$ is the unique, torsion - free covariant differential compatible with $q_{a b}$ and $R$ is the curvature scalar associated with $q_{a b}$.

The reason for the occurence of the Lagrange multipliers $\lambda, \lambda^{a}$ is that the Lagrangean ([.2.1.6) is singular, that is, one cannot solve all the velocities in terms of momenta and therefore one must use Dirac's procedure [20] for the Legendre transform of singular Lagrangeans. In this case the singularity structure is such that the momenta conjugate to $N, U^{a}$ vanish identically, whence the Lagrange multipliers which when varied give the equations of motion $P=P_{a}=0$. The equations of motion with respect to the Hamiltonian (i.e. $\dot{F}:=\{H, F\}$ for any functional $F$ of the canonical coordinates)

$$
H=\int d^{3} x\left[\lambda P+\lambda^{a} P_{a}+U^{a} V_{a}+N C\right]
$$

for $N, U^{a}$ reveal that $N, U^{a}$ are themselves Lagrange multipliers, i.e. completely unspecified functions (proportional to $\lambda, \lambda^{a}$ ) while the equations of motion for $P, P_{a}$ give $\dot{P}=-C, \dot{P}_{a}=-V_{a}$. Since $P, P_{a}$ are supposed to vanish, this requires $C=V_{a}=0$ as well. Thus we see that the Hamiltonian is constrained to vanish in GR ! We will see that this is a direct consequence of the four dimensional diffeomorphism invariance of the theory.

Now the equations of motion for $q_{a b}, P^{a b}$ imply the so-called Dirac (or hypersurface deformation) algebra

$$
\begin{aligned}
\left\{V(U), V\left(U^{\prime}\right)\right\} & =\kappa V\left(\mathcal{L}_{U} U^{\prime}\right) \\
\{V(U), C(N)\} & =\kappa C\left(\mathcal{L}_{U} N\right) \\
\left\{C(N), C\left(N^{\prime}\right)\right\} & =\kappa V\left(q^{-1}\left(N d N^{\prime}-N^{\prime} d N\right)\right)
\end{aligned}
$$


where e.g. $C(N)=\int d^{3} x N C$. These equations tell us that the condition $H=V_{a}=0$ is preserved under evolution, in other words, the evolution is consistent ! This is a non-trivial result. One says, the Hamiltonian and vector constraint form a first class constraint algebra. This algebra is much more complicated than the more familiar Kac-Moody algebras due to the fact that it is not an (infinite) dimensional Lie algebra in the true sense of the word because the "structure constants" on the right hand side of the last line in (I.2.1.12) are not really constants, they depend on the phase space. Such algebras are open in the the terminology of BRST [21] and about their representation theory only very little is known.

\section{Exercise I.2.2.}

Derive (I.2.1.12) from (I.2.1.9).

Hint:

Show first that the Poisson bracket between local functions which contain spatial derivatives is simply the spatial derivatives applied to the Poisson bracket. Since the Poisson bracket of local functions is distributional recall that derivatives of distributions are defined through an integration by parts.

Since the variables $P, P_{a}$ drop out completely from the analysis and $N, U^{a}$ are Lagrange multipliers, we may replace ([.2.1.7) by

$$
S=\frac{1}{\kappa} \int_{\mathbb{R}} d t \int_{\sigma} d^{3} x\left\{\dot{q}_{a b} P^{a b}-\left[U^{a} V_{a}+N H\right]\right\}
$$

with the understanding that $N, U^{a}$ are now completely arbitrary functions which parameterize the freedom in choosing the foliation. Since the Hamiltonian of GR depends on the completely unspecified functions $N, U^{a}$, the motions that it generates in the phase space $\mathcal{M}$ coordinatized by $\left(P^{a b}, q_{a b}\right)$ subject to the Poisson brackets ([.2.1.9) are to be considered as pure gauge transformations. The infinitesimal flow (or motion) of the canonical coordinates generated by the corresponding Hamiltonian vector fields on $\mathcal{M}$ has the following form for an arbitrary tensor $t_{a b}$ built from $q_{a b}, P^{a b}$

$$
\begin{array}{r}
\left\{V(U), t_{a b}\right\}_{E O M}=\kappa\left(\mathcal{L}_{U} t_{a b}\right) \\
\left\{C(N), t_{a b}\right\}_{E O M}=\kappa\left(\mathcal{L}_{N n} t_{a b}\right)
\end{array}
$$

where the subscript EOM means that these relations hold for generic functions on $\mathcal{M}$ only when the vacuum equations of motion (EOM) $R_{\mu \nu}^{(4)}-R^{(4)} g_{\mu \nu} / 2=0$ hold. Equation (1.2.1.14) reveals that $\operatorname{Diff}(M)$ is implemented also in the canonical formalism, however, in a rather non-trivial way: The gauge motions generated by the constraints can be interpreted as four-dimensional diffeomorphisms only when the EOM hold. This was to be expected because a diffemorphism orthogonal to the hypersurface means evolution in the time parameter, what is surprising though is that this evolution is considered as a gauge transformation in GR. Off the solutions, the constraints generate different motions, in other words, the set of gauge symmetries is not $\operatorname{Diff}(M)$ everywhere in the phase space. This is not unexpected: The action (I.2.1.6) is obviously Diff $(M)$ invariant, but so would be any action that is an integral over a four-dimensional scalar density of weight one formed from polynomials in the curvature tensor and its covariant derivatives. This symmetry is completely insensitive to the specific Lagrangean in question, it is kinematical. The dynamics generated by a specific Lagrangean must depend on that Lagrangean, otherwise all Lagrangeans underlying four dimensionally diffeomorphism invariant actions would equal each other up to a diffeomorphism which is certainly not the case (consider for instance higher derivative theories). In particular, that dynamics is, a priori, completely independent of $\operatorname{Diff}(M)$. As a consequence, Dirac observables, that is, functions on $\mathcal{M}$ which are gauge invariant (have vanishing Poisson brackets with the constraints), are not simply functionals of 


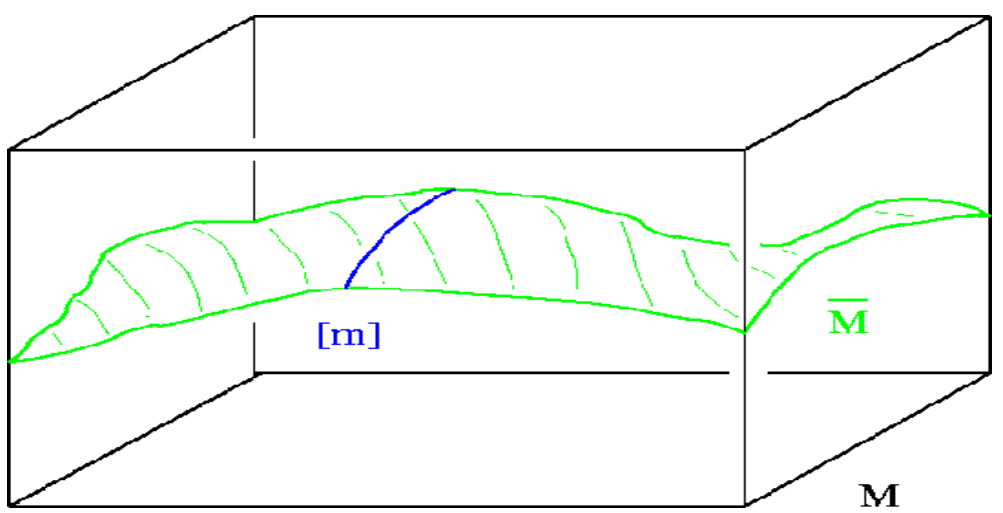

Figure 6: Constraint hypersurface $\overline{\mathcal{M}}$ and gauge orbit $[m]$ of $m \in \overline{\mathcal{M}}$ in $\mathcal{M}$

the four metric invariant under four diffeomorphisms because they must depend on the Lagrangean. The set of these dynamics dependent gauge transformations does not obviously form a group as has been investigated by Bergmann and Komar [22]. The geometrical origin of the hypersurface deformation algebra has been investigated in [23]. Torre and Anderson have shown that for compact $\sigma$ there are no Dirac observables which depend on only a finite number of spatial derivatives of the canonical coordinates [24] which means that Dirac observables will be highly non-trivial to construct.

Let us summarize the gauge theory of GR in figure 6: The constraints $C=V_{a}=0$ define a constraint hypersurface $\overline{\mathcal{M}}$ within the full phase space $\mathcal{M}$. The gauge motions are defined on all of $\mathcal{M}$ but they have the feature that they leave the constraint hypersurface invariant, and thus the orbit of a point $m$ in the hypersurface under gauge transformations will be a curve or gauge orbit $[m]$ entirely within it. The set of these curves defines the so-called reduced phase space and Dirac observables restricted to $\overline{\mathcal{M}}$ depend only on these orbits. Notice that as far as the counting is concerned we have twelve phase space coordinates $q_{a b}, P^{a b}$ to begin with. The four constraints $C, V_{a}$ can be solved to eliminate four of those and there are still identifications under four independent sets of motions among the remaining eight variables leaving us with only four Dirac observables. The corresponding so-called reduced phase space has therefore precisely the two configuration degrees of freedom of general relativity.

\section{I.2.2 Gauge Theory Formulation}

We can now easily introduce the shift from the ADM variables $q_{a b}, P^{a b}$ to the connection variables introduced first by Ashtekar [25] and later somewhat generalized by Immirzi [26] and Barbero [27]. We introduce $s u(2)$ indices $i, j, k, . .=1,2,3$ and co-triad variables $e_{a}^{j}$ with inverse $e_{j}^{a}$ whose relation with $q_{a b}$ is given by

$$
q_{a b}:=\delta_{j k} e_{a}^{j} e_{b}^{k}
$$

Defining the spin connection $\Gamma_{a}^{j}$ through the equation

$$
\partial_{a} e_{b}^{j}-\Gamma_{a b}^{c} e_{c}^{j}+\epsilon_{j k l} \Gamma_{a}^{k} e_{b}^{l}=0
$$

where $\Gamma_{a b}^{c}$ are the Christoffel symbols associated with $q_{a b}$ we now define

$$
A_{a}^{j}=\Gamma_{a}^{j}+\beta K_{a b} e_{j}^{b}, \quad E_{j}^{a}=\sqrt{\operatorname{det}(q)} e_{j}^{a} / \beta
$$


where $\beta \in \mathbb{C}-\{0\}$ is called the Immirzi parameter. In this article we only consider real valued and positive $\beta$. Finally we introduce the $S U(2)$ Gauss constraint

$$
G_{j}:=\partial_{a} E_{j}^{a}+\epsilon_{j k l} A_{a}^{k} E_{l}^{b}
$$

with $\epsilon_{j k l}$ the structure constants of $s u(2)$ which we would encounter in the canonical formulation of any $S U(2)$ gauge theory. As one can check, modulo $G_{j}=0$ one can then write $C, V_{a}$ in terms of $A, E$ as follows

$$
\begin{aligned}
V_{a} & =F_{a b}^{j} E_{j}^{b}, \\
C & =\frac{F_{a b}^{j} \epsilon_{j k l} E_{j}^{a} E_{l}^{b}}{\sqrt{|\operatorname{det}(E)|}}+\text { More }
\end{aligned}
$$

where $F=2(d A+[A, A])$ is the curvature of $A$ and "More" is an additional term which is more complicated but can be treated by similar methods as the one displayed.

We then have the following theorem [25].

\section{Theorem I.2.1.}

Consider the phase space $\mathcal{M}$ coordinatized by $\left(A_{a}^{j}, E_{j}^{b}\right)$ with Poisson brackets

$$
\left\{E_{j}^{a}(x), E_{k}^{b}(y)\right\}=\left\{A_{a}^{j}(x), A_{b}^{k}(y)\right\}=0, \quad\left\{E_{j}^{a}(x), A_{b}^{k}(y)\right\}=\kappa \delta_{b}^{a} \delta_{j}^{k} \delta(x, y)
$$

and constraints $G_{j}, C, V_{a}$. Then, solving only the constraint $G_{j}=0$ and determining the Dirac observables with respect to it leads us back to the $A D M$ phase space with constraints $C, V_{a}$.

The proof of the theorem is non-trivial and tedious and can be found in the notation used here in [1]. Alternatively one can find directions for a proof in the subsequent exercise. In particular, this works only because the Gauss constraint is in evolution with itself and the other constraints, specifically $\{G, G\} \propto G,\{G, V\}=\{G, H\}=0$.

\section{Exercise I.2.3.}

i)

Prove theorem I.2.1.

Hint:

Express $q_{a b}, P^{a b}$ in terms of $A_{a}^{j}, E_{j}^{a}$ by using (I.2.2.1), (I.2.2.2), (I.2.2.3), and (I.2.2.4) and check that the Poisson brackets, with respect to (1.2.2.6), among the solutions $q_{a b}=s_{a b}[A, E], P^{a b}=S^{a b}[A, E]$ equal precisely (I.2.1.9) modulo terms proportional to $G_{j}$.

ii)

Define $G(\Lambda):=\int_{\sigma} d^{3} x \Lambda^{j} G_{j}$ and $D(U):=\int_{\sigma} U^{a}\left[V_{a}-A_{a}^{j} G_{j}\right]$ and $\left[\Lambda, \Lambda^{\prime}\right]_{j}=\epsilon_{j k l} \Lambda^{k}\left(\Lambda^{\prime}\right)^{l}$. Verify the following Poisson brackets

$$
\begin{aligned}
\left\{G(\Lambda), G\left(\Lambda^{\prime}\right)\right\} & =\kappa G\left(\left[\Lambda, \Lambda^{\prime}\right]\right) \\
\{G(\Lambda), V(U)\} & =0 \\
\left\{D(U), D\left(U^{\prime}\right)\right\} & =\kappa D\left(\left[U, U^{\prime}\right]\right)
\end{aligned}
$$

and conclude that the Hamiltonian vector fields of $G(\Lambda)$ and $D(U)$ respectively generate $S U(2)$ gauge transformations and spatial diffeomorphisms of $\sigma$ respectively. 
Hint:

Show first that

$$
\begin{aligned}
\left\{G(\Lambda / \kappa), A_{a}^{j}(x)\right\} & =-\Lambda_{, a}^{j}+\epsilon_{j k l} \Lambda^{k} A_{a}^{l} \\
\left\{D(U / \kappa), A_{a}^{j}(x)\right\} & =U^{b} A_{a, b}^{j}+U_{, a}^{b} A_{b}^{j}
\end{aligned}
$$

to conclude that $A$ transforms as a connection under infinitesimal gauge transformations and as a one-form under infinitesimal diffeomorphisms. Consider then $g_{t}(x):=\exp \left(t \Lambda^{j} \tau_{j} /(2 \kappa)\right)$ and $\varphi_{t}(x):=$ $c_{U, x}(t)$ where $t \mapsto c_{U, x}(t)$ is the unique integral curve of $U$ through $x$, that is, $\dot{c}_{U, x}(t)=U\left(c_{U, x}(t)\right), c_{U, x}(0)=$ $x$. Recall that the usual transformation behaviour of connections and one-forms under finite gauge transformations and diffeomorphisms respectively is given by (e.g. 28/)

$$
\begin{aligned}
& A^{g}=-d g g^{-1}+A d_{g}(A) \\
& A^{\varphi}=\varphi^{*} A
\end{aligned}
$$

where $A=A_{a}^{j} d x^{a} \tau_{j} / 2, A d_{g}()=.g(.) g^{-1}$ denotes the adjoint representation of $S U(2)$ on $s u(2)$ and $\varphi^{*}$ denotes the pull-back map of $p$-forms and $i \tau_{j}$ are the Pauli matrices so that $\tau_{j} \tau_{k}=-\delta_{j k} 1_{2}+\epsilon_{j k l} \tau_{l}$. Verify then that (1.2.2.8) is the derivative at $t=0$ of (1.2.2.9) with $g:=g_{t}, \varphi:=\varphi_{t}$. Similarly, derive that $E$ transforms as an su(2)-valued vector field of density weight one. (Recall that a tensor field $t$ of some type is said to be of density weight $r \in \mathbb{R}$ if $t \sqrt{|\operatorname{det}(s)|}^{-r}$ is an ordinary tensor field of the same type where $s_{a b}$ is any non-degenerate symmetric tensor field).

From the point of view of the classical theory we have made things more complicated: Instead of twelve variables $q, P$ we now have eighteen $A, E$. However, the additional six degrees of freedom are removed by the first class Gauss constraint which shows that working on our gauge theory phase space is equivalent to working on the ADM phase space. The virtue of this extended phase space is that canonical GR can be formulated in the language of a canonical gauge theory where $A$ plays the role of an $S U(2)$ connection with canonically conjugate electric field $E$. Besides the remark that this fact could be the starting point for a possible gauge group unification of all four forces we now have access to a huge arsenal of techniques that have been developed for the canonical quantization of gauge theories. It is precisely this fact that has enabled steady progress in this field in the last fifteen years while one was stuck with the ADM formulation for almost thirty years.

\section{I.3 Canonical Quantization Programme for Theories with Constraints}

\section{I.3.1 Refined Algebraic Quantization (RAQ)}

As we have seen, GR can be formulated as a constrained Hamiltonian system with first class constraints. The quantization of such systems has been considered first by Dirac 20 and was later refined by a number of authors. It is now known under the name refined algebraic quantization (RAQ). We will briefly sketch the main ideas following [29].

i) Phase Space and Constraints

The starting point is a phase space $(\mathcal{M},\{.,\}$.$) together with a set of first class constraints C_{I}$ and possibly a Hamiltonian $H$. 
ii) Choice of Polarization

In order to quantize the phase space we must choose a polarization, that is, a Lagrangean submanifold $\mathcal{C}$ of $\mathcal{M}$ which is called configuration space. The coordinates of $\mathcal{C}$ have vanishing Poisson brackets among themselves. If $\mathcal{M}$ is a cotangent bundle, that is, $\mathcal{M}=T^{*} \mathcal{Q}$ then it is natural to choose $\mathcal{Q}=\mathcal{C}$ and we will assume this to be the case in what follows. For more general cases, e.g. compact phases spaces one needs ideas from geometrical quantization, see e.g. [30. The idea is that (generalized, see below) points of $\mathcal{C}$ serve as arguments of the vectors of the Hilbert space to be constructed.

iii) Preferred Kinematical Poisson Subalgebra

Consider the space $C^{\infty}(\mathcal{C})$ of smooth functions on $\mathcal{C}$ and the space $V^{\infty}(\mathcal{C})$ of smooth vector fields on $C$. The vertical polarization of $\mathcal{M}$, that is, the space of fibre coordinates called momentum space, generates preferred elements of $V^{\infty}(\mathcal{C})$ through $\left(v_{p}[f]\right)(q):=(\{p, f\})(q)$ where we have denoted configuration and momentum coordinates by $q, p$ respectively and $v[f]$ denotes the action of a vector field on a function. The pair $C^{\infty}(\mathcal{C}) \times \mathcal{V}^{\infty}(\mathcal{C})$ forms a Lie algebra defined by $\left[(f, v),\left(f^{\prime}, v^{\prime}\right)\right]=\left(v\left[f^{\prime}\right]-v^{\prime}[f],\left[v, v^{\prime}\right]\right)$ of which the algebra $\mathcal{B}$ generated by elements of the form $\left(f, v_{p}\right)$ forms a subalgebra. We assume that $\mathcal{B}$ is closed under complex conjugation which becomes its * -operation (involution).

iv) Representation Theory of the Corresponding Abstract* ${ }^{*}$ Algebra

We are looking for all irreducible ${ }^{*}$-representations $\pi: \mathcal{B} \rightarrow \mathcal{L}\left(\mathcal{H}_{\text {kin }}\right)$ of $\mathcal{B}$ as linear operators on a kinematical Hilbert space $\mathcal{H}_{\text {kin }}$ such that the ${ }^{*}$-relations becomes the operator adjoint and such that the canonical commutation relations are implemented, that is, for all $a, b \in \mathcal{B}$

$$
\begin{aligned}
\pi(a)^{\dagger} & =\pi\left(a^{*}\right) \\
{[\pi(a), \pi(b)] } & =i \hbar \pi([a, b])
\end{aligned}
$$

Strictly speaking, (I.3.1.1) is to be supplemented by the domains on which the operators are defined. In order to avoid this one will work with the subalgebra of $C^{\infty}(\mathcal{C})$ formed by bounded functions, say of compact support and one will deal with exponentiated vector fields in order to obtain bounded operators. Irreducibility is a physically meaningful requirement because we are not interested in Hilbert spaces with superselection sectors and the reason for why we do not require the full Poisson algebra to be faithfully represented is that this is almost always impossible in irreducible representations as stated in the famous Groenewald - van Hove theorem. The Hilbert space that one gets can usually be described in the form $L_{2}(\overline{\mathcal{C}}, d \mu)$ where $\overline{\mathcal{C}}$ is a distributional extension of $\mathcal{C}$ and $\mu$ is a probability measure thereon. A well-known example is the case of free scalar fields on Minkowski space where $\mathcal{C}$ is some space of smooth scalar fields on $\mathbb{R}^{3}$ vanishing at spatial infinity while $\overline{\mathcal{C}}$ is the space of tempered distributions on $\mathbb{R}^{3}$ and $\mu$ is a normalized Gaussian measure on $\overline{\mathcal{C}}$.

v) Selection of Suitable Kinematical Representations

Certainly we want a representation which supports also the constraints and the Hamiltonian as operators which usually will limit the number of available representations to a small number, if possible at all. The constraints usually are not in $\mathcal{B}$ unless linear in momentum and the expressions $\hat{C}_{I}:=\pi\left(C_{I}\right), \hat{H}=\pi(H)$ will involve factor ordering ambiguities as well as regularizationand renormalization processes in the case of field theories. In the generic case, $\hat{C}_{I}, \hat{H}$ will not be bounded and $\hat{C}_{I}$ will not be symmetric. We will require that $\hat{H}$ is symmetric and that the constraints are at least closable, that is, they are densely defined together with their 
adjoints. It is then usually not too difficult to find a dense domain $\mathcal{D}_{\text {kin }} \subset \mathcal{H}_{\text {kin }}$ on which all these operators and their adjoints are defined and which they leave invariant. Typically $\mathcal{D}_{k i n}$ will be a space of smooth functions of rapid decrease so that arbitray derivatives and polynomials of the configuration variables are defined on them and such spaces naturally come with their own topology which is finer than the subspace topology induced from $\mathcal{H}_{\text {kin }}$ whence we have a topological inclusion $\mathcal{D}_{\text {kin }} \hookrightarrow \mathcal{H}_{\text {kin }}$.

\section{vi) Imposition of the Constraints}

The two step process in the classical theory of solving the constraints $C_{I}=0$ and looking for the gauge orbits is replaced by a one step process in the quantum theory, namely looking for solutions $l$ of the equations $\hat{C}_{I} l=0$. This is because it is obviously solves the constraint at the quantum level (in the corresponding representation on the solution space the constraints are replaced by the zero operator) and it simultaneously looks for states that are gauge invariant because $\hat{C}_{I}$ is the quantum generator of gauge transformations.

Now, unless the point $\{0\}$ is in the common point spectrum of all the $\hat{C}_{I}$, solutions $l$ to the equations $\hat{C}_{I} l=0 \forall I$ do not lie in $\mathcal{H}_{k i n}$, rather they are distributions. Here one has several options, one could look for solutions in the space $\mathcal{D}_{k i n}^{\prime}$ of continuous linear functionals on $\mathcal{D}_{\text {kin }}$ (topological dual) or in the space $\mathcal{D}_{k i n}^{*}$ of linear functionals on $\mathcal{D}_{k i n}$ with the topology of pointwise convergence (algebraic dual). Since certainly $\mathcal{H}_{\text {kin }} \subset \mathcal{D}_{\text {kin }}^{\prime} \subset \mathcal{D}_{\text {kin }}^{*}$ let us choose the latter option for the sake of more generality. The topology on $\mathcal{H}_{k i n}$ is again finer than the subspace topology induced from $\mathcal{D}_{k i n}^{*}$ so that we obtain a Gel'fand triple or Rigged Hilbert Space

$$
\mathcal{D}_{\text {kin }} \hookrightarrow \mathcal{H}_{\text {kin }} \hookrightarrow \mathcal{D}_{\text {kin }}^{*}
$$

This a slight abuse of terminology since the name is usually reserved for the case that $\mathcal{D}_{k i n}$ carries a nuclear topology (generated by a countable family of seminorms separating the points) and that $\mathcal{D}_{\text {kin }}^{*}$ is its topological dual.

We are now looking for a subspace $\mathcal{D}_{\text {phys }}^{*} \subset \mathcal{D}_{\text {kin }}^{*}$ such that for its elements $l$ holds

$$
\left[\hat{C}_{I}^{\prime} l\right](f):=l\left(\hat{C}_{I}^{\dagger} f\right)=0 \forall f \in \mathcal{D}_{k i n}, \forall I
$$

The prime on the left hand side of this eqution defines a dual, anti-linear representation of the constraints on $\mathcal{D}_{k i n}^{*}$. The reason for the adjoint on the right hand side of this equation is that if $l$ would be an element of $\mathcal{H}_{k i n}$ then ([.3.1.3) would be replaced by

$$
\left[\hat{C}_{I}^{\prime} l\right](f):=<\hat{C}_{I} l, f>_{k i n}=<l, \hat{C}_{I}^{\dagger} f>_{k i n}=: l\left(\hat{C}_{I}^{\dagger} f\right) \forall f \in \mathcal{D}_{k i n}, \forall I
$$

where $\langle., .\rangle_{\text {kin }}$ denotes the kinematical inner product, so that (I.3.1.3) is the natural extension of ([.3.1.4) from $\mathcal{H}_{\text {kin }}$ to $\mathcal{D}_{\text {kin }}^{*}$

vii) Anomalies

Since we have a first class constraint algebra, we know that classically $\left\{C_{I}, C_{J}\right\}=f_{I J}{ }^{K} C_{K}$ for some structure functions $f_{I J}{ }^{K}$ which depend in general on the phase space point $m \in \mathcal{M}$. The translation of this equation into quantum theory is then plagued with ordering ambiguities, because the structure functions turn into operators as well. It may therefore happen that, e.g.

$$
\left[\hat{C}_{I}, \hat{C}_{J}\right]=i \hbar \hat{C}_{K} \hat{f}_{I J}{ }^{K}=i \hbar\left\{\left[\hat{C}_{K}, \hat{f}_{I J}{ }^{K}\right]+\hat{f}_{I J}{ }^{K} \hat{C}_{K}\right\}
$$

and it follows that any $l \in \mathcal{D}_{\text {phys }}^{*}$ also solves the equation $\left(\left[\hat{C}_{K}, \hat{f}_{I J}{ }^{K}\right]\right)^{\prime} l=0$ for all $I, J$. If that commutator is not itself a constraint again, then it follows that $l$ solves more than only the 
equations $\hat{C}_{I}^{\prime} l=0$ and thus the quantum theory has less physical degrees of freedom than the classical theory. This situation, called an anomaly, must be avoided by all means.

viii) Dirac Observables and Physical Inner Product

Since generically $\mathcal{H}_{\text {kin }} \cap \mathcal{D}_{\text {phys }}^{*}=\emptyset$, the space $\mathcal{D}_{\text {phys }}^{*}$ cannot be equipped with the scalar product $<.,>_{k i n}$. It is here wehere Dirac observables come into play. A strong Dirac observable is an operator $\hat{O}$ on $\mathcal{H}_{\text {kin }}$ which is, together with its adjoint, densely defined on $\mathcal{D}_{\text {kin }}$ and which commutes with all constraints, that is, $\left[\hat{O}, \hat{C}_{I}\right]=0$ for all $I$. We require that $\hat{O}$ is the quantization of a real valued function $O$ on the phase space and the condition just stated is the quantum version of the classical gauge invariance condition $\left\{O, C_{I}\right\}=0$ for all $I$. A weak Dirac observable is the quantum version of the more general condition $\left\{O, C_{I}\right\}_{\mid C_{J}}=0 \forall J=0 \forall I$ and simply means that the space of solutions is left invariant by the natural dual action of the operator $\hat{O}^{\prime} \mathcal{D}_{\text {phys }}^{*} \subset \mathcal{D}_{\text {phys }}^{*}$.

A physical inner product on a subset $\mathcal{H}_{\text {phys }} \subset \mathcal{D}_{\text {phys }}^{*}$ is a positive definite sesquilinear form $<.$, . $>_{\text {phys }}$ with respect to which the $\hat{O}^{\prime}$ become self-adjoint operators, that is, $\hat{O}^{\prime}=\left(\hat{O}^{\prime}\right)^{\star}$ where the adjoint on $\mathcal{H}_{\text {phys }}$ is denoted by $\star$. Notice that $\left[\hat{O}_{1}^{\prime}, \hat{O}_{2}^{\prime}\right]=\left(\left[\hat{O}_{1}, \hat{O}_{2}\right]\right)^{\prime}$ so that commutation relations on $\mathcal{H}_{\text {kin }}$ are automatically transferred to $\mathcal{H}_{\text {phys }}$ which then carries a proper *-representation of the physical observables. The observables themselves will only be defined on a dense domain $\mathcal{D}_{\text {phys }} \subset \mathcal{H}_{\text {phys }}$ and we get a second Gel'fand triple

$$
\mathcal{D}_{\text {phys }} \hookrightarrow \mathcal{H}_{\text {phys }} \hookrightarrow \mathcal{D}_{\text {phys }}^{*}
$$

In fortunate cases, for instance when the $\hat{C}_{I}$ are mutually commuting self-adjoint operators on $\mathcal{H}_{\text {kin }}$, all we have said is just a fancy way of stating the fact that $\mathcal{H}_{\text {kin }}$ has a direct integral decomposition

$$
\mathcal{H}_{\text {kin }}=\int_{S}^{\oplus} d \nu(\lambda) \mathcal{H}_{\lambda}
$$

over the spectrum $S$ of the constraint algebra with a measure $\nu$ and eigenspaces $\mathcal{H}_{\lambda}$ which are left invariant by the strong observables and therefore $\mathcal{H}_{\text {phys }}=\mathcal{H}_{0}$. In the more general cases that are of concern to us, more work is required.

ix) Classical Limit

It is by no means granted that the representation $\mathcal{H}_{\text {phys }}$ that one finally arrived at, carries semiclassical states, that is states $\psi_{[m]}$ labelled by gauge equivalence classes $[m]$ of points $m \in \mathcal{M}$ with respect to which the Dirac observables have the correct expectation values and with respect to which their relative fluctuations are small, that is, roughly speaking

$$
\left|\frac{<\psi_{[m]}, \hat{O}^{\prime} \psi_{[m]}>_{p h y s}}{O(m)}-1\right| \ll 1 \text { and }\left|\frac{<\psi_{[m]},\left(\hat{O}^{\prime}\right)^{2} \psi_{[m]}>_{p h y s}}{\left(<\psi_{[m]}, \hat{O}^{\prime} \psi_{[m]}>_{p h y s}\right)^{2}}-1\right| \ll 1
$$

Only when such a phase exists are we sure that we have not constructed some completely spurious sector of the quantum theory which does not admit the correct classical limit.

\section{I.3.2 Selected Examples with First Class Constraints}

In the case that a theory has only first class constraints, Dirac's algorithm [20] boils down to the following four steps: 
1)

Define the momentum $p_{a}$ conjugate to the configuration variable $q^{a}$ by (Legendre transform)

$$
p_{a}:=\partial S / \partial \dot{q}^{a}
$$

where $S$ is the action.

2)

Equation ([.3.2.1) defines $p_{a}$ as a function of $q^{a}, \dot{q}^{a}$ and if it is not invertible to define the $\dot{q}^{a}$ as a function of $q^{a}, p_{a}$ we get a collection of so-called primary constraints $C_{I}$, that is, identities among the $q^{a}, p_{a}$. In this situation one says that $S$ or the Lagrangean is singular.

3)

Using that $q^{a}, p_{a}$ have canonical Poisson brackets, compute all possible Poisson brackets $C_{I J}:=$ $\left\{C_{I}, C_{J}\right\}$. If some $C_{I_{0} J_{0}}$ is not zero when all $C_{K}$ vanish, then add this $C_{I_{0} J_{0}}$, called a secondary constraint, to the set of primary constraints.

4)

Iterate 3) until the $C_{I}$ are in involution, that is, no new secondary constraints appear.

In this report we will only deal with theories which have no second class constraints, so this algorithm is all we need.

\section{Exercise I.3.1.}

Perform the quantization programme for a couple of simple systems in order to get a feeling for the formalism:

1. Momentum Constraint

$\mathcal{M}=T^{*} \mathbb{R}^{2}$ with standard Poisson brackets among $q^{a}, p_{a} ; a=1,2$ and constraint $C:=p_{1}$. Choose $\mathcal{H}_{\text {kin }}=L_{2}\left(\mathbb{R}^{2}, d^{2} x\right), \mathcal{D}_{\text {kin }}=\mathcal{S}\left(\mathbb{R}^{2}\right), \mathcal{D}_{\text {kin }}^{*}=\mathcal{S}^{\prime}\left(\mathbb{R}^{2}\right)$ (spaces of functions of rapid decrease and tempered distributions respectively).

Solution:

Dirac observables are the conjugate pair $q^{2}, p_{2}, \mathcal{H}_{\text {phys }}=L_{2}\left(\mathbb{R}, d x_{2}\right)$.

Hint: Work in the momentum representation and conclude that the general solution is of the form $l_{f}\left(p_{1}, p_{2}\right)=\delta\left(p_{1}\right) f\left(p_{2}\right)$ for $f \in \mathcal{S}^{\prime}(\mathbb{R})$.

2. Angular Momentum Constraint

$\mathcal{M}=T^{*} \mathbb{R}^{3}$ with standard Poisson brackets among $q^{a}, p_{a} ; a=1,2,3$ and constraints $C_{a}:=$

$\epsilon_{a b c} x^{b} p_{c}$. Check the first class property and choose the kinematical spaces as above with $\mathbb{R}^{2}$ replaced by $\mathbb{R}^{3}$.

Solution:

Dirac observables are the conjugate pair $r:=\sqrt{\delta_{a b} q^{a} q^{b}} \geq 0, p_{r}=\delta_{a b} q^{a} p_{b} / r$, the physical phase space is $T^{*} \mathbb{R}_{+}$and $\mathcal{H}_{\text {phys }}=L_{2}\left(\mathbb{R}_{+}, r^{2} d r\right)$ where $\hat{r}$ is a multiplication operator and $\hat{p}_{r}=i \hbar \frac{1}{r} \frac{d}{d r} r$ with dense domain of symmetry given by the square integrable functions $f$ such that $f$ is regular at $r=0$.

Hint:

Introduce polar coordinates and decompose kinematical wave functions into spherical harmonics. Conclude that the physical Hilbert space this time is just the restriction of the kinematical Hilbert space to the zero angular momentum subspace, that is, $\mathcal{H}_{\text {phys }} \subset \mathcal{H}_{\text {kin }}$. The reason is of course that the spectrum of the $\hat{C}_{a}$ is pure point (discrete).

3. Relativistic Particle

Consider the Lagrangean $L=-m \sqrt{-\eta_{\mu \nu} \dot{q}^{\mu} \dot{q}^{\nu}}$ where $m$ is a mass parameter, $\eta$ is the Minkowski 
metric and $\mu=0,1, . ., D$. Verify that the Lagrangean is singular, that is, the velocities $\dot{q}^{\mu}$ cannot be expressed in terms of the momenta $p_{\mu}=\partial L / \partial \dot{q}^{\mu}$ which gives rise to the mass shell constraint $C=m^{2}+\eta^{\mu \nu} p_{\mu} p_{\nu}$. Verify that this happens because the corresponding action is invariant under Diff( $(\mathbb{R})$, that is, reparameterizations $t \mapsto \varphi(t), \dot{\varphi}(t)>0$. Perform the Dirac analysis for constraints and conclude that the system has no Hamiltonian, just the Hamiltonian constraint $C$ which generates reparameterizations on the kinematical phase space $\mathcal{M}=T^{*} \mathbb{R}^{D+1}$ with standard Poisson brackets. Now choose kinematical spaces as in 1 . with $\mathbb{R}^{2}$ replaced by $\mathbb{R}^{D+1}$.

Solution:

Conjugate Dirac observables are $Q^{a}=q^{a}-\frac{q^{0} p_{a}}{\sqrt{m^{2}+\delta^{a b} p_{a} p_{b}}}$ and $\mathcal{H}_{\text {phys }}=L_{2}\left(\mathbb{R}^{D}, d^{D} p\right)$ on which $\hat{q}^{0}=0$.

Hint:

Work in the momentum representation and conclude that the general solution to the constraints is of the form $l_{f}=\delta(C) f\left(p_{0}, \vec{p}\right)$. Now notice that the $\delta$-distribution can be written as a sum of two $\delta$-distribution corresponding to the positive and negative mass shell and choose $f$ to have support in the former.

This example has features rather close to those of general relativity.

4. Maxwell Theory

Consider the action for free Maxwell-theory on Minkowski space and perform the Legendre transform. Conclude that there is a first class constraint $C=\partial_{a} E^{a}$ (Gauss constraint) with Lagrange multiplier $A_{0}$ and a Hamiltonian $H=\int_{\mathbb{R}^{3}} d^{3} x\left[E^{a} E^{b}+B^{a} B^{b}\right] / 2$ where $E^{a}=\dot{A}_{a}-\partial_{a} A_{0}$ is the electric field and $B^{a}=\epsilon^{a b c} \partial_{b} A_{c}$ the magnetic one. Verify that the Gauss constraint generates $U(1)$ gauge transformations $A \mapsto A-d f$ while $E^{a}$ is gauge invariant. Choose $\mathcal{H}_{\text {kin }}$ to be the standard Fock space for three massless, free scalar fields $A_{a}$ and as $\mathcal{D}_{\text {kin }}, \mathcal{D}_{\text {kin }}^{*}$ the finite linear span of $n$-particle states and its algebraic dual respectively.

Solution:

Conjugate Dirac observables are the transversal parts of $A, E$ respectively, e.g. $E_{\perp}^{a}=E^{a}-$ $\partial_{a} \frac{1}{\Delta} \partial_{b} E^{b}$ where $\Delta$ is the Laplacian on $\mathbb{R}^{3}$. The physical Hilbert space is the standard Fock space for two free, masless scalar fields corresponding to these transversal degrees of freedom.

Hint:

Fourier transform the fields and compute the standard annihilation and creation operators $\hat{z}_{a}(k), \hat{z}_{a}^{\dagger}(k)$ with canonical commutation relations. Express the Gauss constraint operator in terms of them and conclude that the gauge invariant part satisfies $\hat{z}_{a}(k) k^{a}=0$. Introduce $\hat{z}_{I}(k)=\hat{z}_{a}(k) e_{I}^{a}(k)$ where $\vec{e}_{1}(k), \vec{e}_{2}(k), \vec{e}_{3}(k):=\vec{k} /\|k\|$ form an oriented orthonormal basis. Conclude that physical states are states without longitudonal excitations and build the Fock space generated by the $\hat{z}_{1}^{\dagger}(k), \hat{z}_{2}^{\dagger}(k)$ from the kinematical vacuum state. 


\section{Part II}

\section{Mathematical and Physical Foundations of Quantum General Relativity}




\section{II.1 Mathematical Foundations}

\section{II.1.1 Polarization and Preferred Poisson Algebra $\mathcal{B}$}

The first two steps of the quantization programme were already completed in section [.2: The phase space $\mathcal{M}$ is coordinatized by canonically conjugate pairs $\left(A_{a}^{j}, E_{j}^{a}\right)$ where $A$ is an $S U(2)$ connection over $\sigma$ while $E$ is a su(2)-valued vector density of weight one over $\sigma$ and the Poisson brackets were displayed in ([.2.2.6). Strictly speaking, since $\mathcal{M}$ is an infinite dimensional space, one must supply $\mathcal{M}$ with a manifold structure modelled on some Banach space but we will skip these functional analytic niceties here, see [1] for further information. Also we must specify the principal fibre bundle of which $A$ is the pull-back by local sections of a globally defined connection, and we must specify the vector bundle associated to that principal bundle under the adjoint representation of which $E$ is the pull-back by local sections. Again, in order not to dive too deeply into fibre bundle theoretic subtleties, we will assume that the principal fibre bundle is trivial so that $A, E$ are actually globally defined. In fact, for the case of $G=S U(2)$ and $\operatorname{dim}(\sigma)=3$ one can show that the fibre bundle is necessarily trivial but for the generalization to the generic case we again refer the reader to [1].

With these remrks out of the way we may begin by defining a polarization. The fact that GR has been casted into the language of a gauge theory suggests the choice $\mathcal{C}=\mathcal{A}$, the space of smooth $S U(2)$ connections over $\sigma$.

The next question then is how to choose the space $C^{\infty}(\mathcal{A})$. Since we are dealing with a field theory, it is not clear a priori what smooth or even differentiable means. In order to give precise meaning to this, one really has to equip $\mathcal{A}$ with a manifold structure modelled on a Banach space. This is because one usually says that a function $F: \mathcal{A} \rightarrow \mathbb{C}$ is differentiable at $A_{0} \in \mathcal{A}$ provided that there exists a bounded linear functional $D F_{A_{0}}: T_{A_{0}}(\mathcal{A}) \rightarrow \mathbb{C}$ such that $F\left[A_{0}+\delta A\right]-F\left[A_{0}\right]-D F_{A_{0}} \cdot \delta A$ vanishes "faster than linearly" for arbitrary tangent vectors $\delta A \in T_{A_{0}}(\mathcal{A})$ at $A_{0}$. (The proper way of saying this is using the natural Banach norm on $T(\mathcal{A})$.) Of course, in physicist's notation the differential $D F_{A_{0}}=(\delta F / \delta A)\left(A_{0}\right)$ is nothing else than the functional derivative. Using this definition it is clear that polynomials in $A_{a}^{j}(x)$ are not differentiable because their functional derivative is proportional to a $\delta$-distribution as it is clear from ([.2.2.6). Thus we see that the smooth functions of $A$ have to involve some kind of smearing of $A$ with test functions, which is generic in field theories.

Now this smearing should be done in a judicious way. The function $F[A]:=\int_{\sigma} d^{3} x F_{j}^{a}(x) A_{a}^{j}(x)$ for some smooth test function $F_{j}^{a}$ of compact support is certainly smooth in the above sense, its functional derivative being equal to $F_{a}^{j}$ (which is a bounded operator if $F$ is e.g. an $L_{2}$ function on $\sigma$ and the norm on the tangent spaces is an $L_{2}$ norm). However, this function does not transform nicely under $S U(2)$ gauge transformations which will make it very hard to construct $S U(2)$ invariant functions from them. Here it helps to look up how physicists have dealt with this problem in ordinary canonical quantum Yang-Mills gauge theories and they found the following, more or less unique solution [31]:

Given a curve $c:[0,1] \rightarrow \sigma$ in $\sigma$ and a point $A \in \mathcal{A}$ we define the holonomy or parallel transport $A(c):=h_{c, A}(1) \in S U(2)$ as the unique solution to the following ordinary differential equation for functions $h_{c, A}:[0,1] \rightarrow S U(2)$

$$
\frac{d h_{c, A}(t)}{d t}=h_{c, A}(t) A_{a}^{j}(c(t)) \frac{\tau_{j}}{2} \dot{c}^{a}(t), \quad h_{c, A}(0)=1_{2}
$$

Exercise II.1.1. Verify that ([I.1.1.1) is equivalent with

$$
A(c)=\mathcal{P} \cdot \exp \left(\int_{c} A\right)=1_{2}+\sum_{n=1}^{\infty} \int_{0}^{t} d t_{1} \int_{t_{1}}^{1} d t_{2} . . \int_{t_{n-1}}^{1} A\left(t_{1}\right) . . A\left(t_{n}\right)
$$


where $\mathcal{P}$ denotes the path ordering symbol which orders the curve parameters from left to right according to their value beginning with the smallest one and $A(t):=A_{a}^{j}(c(t)) \dot{c}^{a}(t) \tau_{j} / 2$.

With this definition it is not difficult to verify the following transformation behaviour of $A(c)$ under gauge transformations and spatial diffeomorphisms respectively (recall ([I.2.2.9)):

$$
A^{g}(c)=g(b(c)) A(c) g(f(c))^{-1} \text { and } A^{\varphi}(c)=A\left(\varphi^{-1}(c)\right)
$$

where $b(c), f(c)$ denote the beginning and final point of a curve respectively. Thus, the behaviour under gauge transformations is extremely simple which makes it easy to construct gauge invariant functions, for instance the Wilson loop functions $\operatorname{Tr}(A(c))$ where $c$ is a closed curve, that is, a loop. This is the reason why QGR is also denoted as Loop Quantum Gravity. That holonomies also transform very naturally under spatial diffeomorphisms as depicted in the second equation of (II.1.1.3) has the following mathematical origin: A connection is in particular a one-form, therefore it is naturally integrated (smeared) over one-dimensional submanifolds of $\sigma$. Here natural means without using a background metric. Now the holonomy is not really the exponential of $\int_{c} A$ but almost as shown in ([I.1.1.2). Thus, holonomies are precisely in accordance with our wish to construct a background independent quantum field theory. Moreover, the simple transformation behaviour under diffeomorphisms again makes it simple to construct spatially diffeomorphism invariant functions of holonomies: These will be functions only labelled by diffeomporphism invariance classes of loops, but these are nothing else than knot classes. Thus QGR has an obvious link with topological quantum field theory (TQFT) [32] which makes it especially attractive and was one of the major motivations for Jacobson, Rovelli and Smolin to consider Wilson loop functions for canonical quantum gravity [33]. Finally one can show [34 that the holonomies separate the points of $\mathcal{A}$, i.e. they encode all the information that is contained in a connection.

The fact that the holonomy smears $A$ only one-dimensionally is nice due to the above reasons but it is also alarming because its functional derivative is certainly distributional and thus does not exist in the strict mathematical sense. However, in order to obtain a well-defined Poisson algebra it is not necessary to have smooth functions of $A$, it is only sufficient. The key idea idea is that if we smear also the electric fields $E$ then we might get a non-distributional Poisson algebra. By inspection from ([.2.2.6) it is clear that $E$ has to be smeared in at least two dimensions in order to achieve this. Now again background independence comes to our help: Let $\epsilon_{a b c}$ be the totally skew, background independent tensor density of weight -1 , that is, $\epsilon_{a b c}=\delta_{[a}^{1} \delta_{b}^{2} \delta_{c]}^{3}$ where [..] denotes total antisymmetrization. Then $(* E)_{a b}^{j}:=E_{a b}^{j}:=E_{j}^{c} \epsilon_{a b c}$ is a 2-form of density weight 0 . Therefore $E$ is naturally smeared in two dimensions. Notice that the smearing dimensions of momenta and configuration variables add up to the dimension of $\sigma$, they are dual to each other which is a generic phenomenon for any canonical theory in any dimension. We are therefore led to consider the electric fluxes

$$
E_{j}(S)=\int_{S} * E_{j}
$$

where $S$ is a two-dimensional, open surface. It is easy to check that $E(S):=E_{j}(S) \tau_{j}$ has the following transformation behaviour

$$
E^{g}(S)=\int_{S} \operatorname{Ad}_{g}(* E) \text { and } E^{\varphi}(S)=E\left(\varphi^{-1}(S)\right)
$$

Thus, while the transformation under spatial diffeomorphisms is again simple, the one under gauge transformations is not. However, the idea is that the $E_{j}(S)$ are the basic building blocks for more 
complicated functions of $E$ which are already gauge invariant. The prototype of such a function is the area functional for a parameterized surface $X_{S}: D \rightarrow \sigma, D \subset \mathbb{R}^{2}$

$$
\operatorname{Ar}(S):=\int_{D} d^{2} u \sqrt{\operatorname{det}\left(X_{S}^{*} q\right)}
$$

Exercise II.1.2. Define $n_{a}^{S}:=\epsilon_{a b c} X_{S, u^{1}}^{b} X_{S, u^{2}}^{c}$ and verify that (II.1.1.6) coincides with

$$
\operatorname{Ar}(S):=\beta \int_{D} d^{2} u \sqrt{\left(E_{j}^{a} n_{a}^{S}\right)\left(E_{j}^{b} n_{b}^{S}\right)}
$$

where $\beta$ is the Immirzi parameter.

It is clear that $E_{j}(S)=\int_{D} d^{2} u E_{j}^{a} n_{a}^{S}$ so that the area functional can be written as the limit of a Riemann sum, over small surfaces that partition $S$, of functions of the electric fluxes for those small surfaces.

Let us see whether the Poisson bracket between an electric flux and a holonomy is well-defined. Actually, let us be slightly more general and introduce the following notion: Let us losely think for the moment of a graph $\gamma$ as a collection of a finite number of smooth, compactly supported, oriented curves, called edges $e$, which intersect at most in their end points, which are called vertices $v$. We denote by $E(\gamma), V(\gamma)$ the edge and vertex set of $\gamma$ respectively. A precise definition will be given in section $\llbracket 1.1 .2$.

\section{Definition II.1.1.}

Given a graph $\gamma$ we define

$$
p_{\gamma}: \mathcal{A} \rightarrow S U(2)^{|E(\gamma)|} ; A \mapsto(A(e))_{e \in E(\gamma)}
$$

A function $f: \mathcal{A} \rightarrow \mathbb{C}$ is said to be cylindrical over a graph $\gamma$, if there exists a function $f_{\gamma}$ : $S U(2)^{|E(\gamma)|} \rightarrow \mathbb{C}$ such that $f=f_{\gamma} \circ p_{\gamma}$. We denote by $C y l_{\gamma}^{n}, n=0,1, . ., \infty$ the set of $n$-times continuously differentiable cylindrical functions over $\gamma$ and by $C y l^{n}$ the set functions which are cylindrical over some $\gamma$ with the same differentiability type. Here we say that $f=f_{\gamma} \circ p_{\gamma} \in C y l_{\gamma}^{n}$ if and only if $f_{\gamma}$ is $n$-times continuously differentiable with respect to the standard differential structure on $S U(2)^{|E(\gamma)|}$.

Our Poisson Algebra will be based on the set of functions $\mathrm{Cy}^{\infty}$ which certainly form an Abelean Poisson subalgebra. Our next task will be to compute the Poisson bracket between a flux and an element of $\mathrm{Cyl}^{\infty}$. In order to compute this we will use the chain rule $\left(f \in \mathrm{Cyl}_{\gamma}^{\infty}\right)$

$$
\left\{E_{j}(S), f\right\}(A)=\sum_{e \in E(\gamma)}\left[\frac{\partial f_{\gamma}\left(\left\{h_{e^{\prime}}\right\}_{e^{\prime} \in E(\gamma)}\right)}{\partial\left(h_{e}\right)_{A B}}\left\{E_{j}(S),\left(h_{e}\right)_{A B}\right\}\right]_{\mid h_{e^{\prime}}=A\left(e^{\prime}\right)}
$$

so that the bracket will be well-defined once the bracket between a holonomy and a flux is welldefined. To compute this the intersection structure of $e$ with $S$ is somewhat important. In order to simplify the notation, we notice that we can always take $\gamma$ to be adapted to $S$, that is, every edge $e$ belongs to one of the following three types:

a) $e \in E_{\text {out }}(\gamma) \Leftrightarrow e \cap S=\emptyset$.

b) $e \in E_{\text {in }}(\gamma) \Leftrightarrow e \cap S=e$.

c) $e \in E_{\text {trans }}(\gamma) \Leftrightarrow e \cap S=b(e)$.

This can be achieved by subdividing edges into a finite number of segments and inverting their 


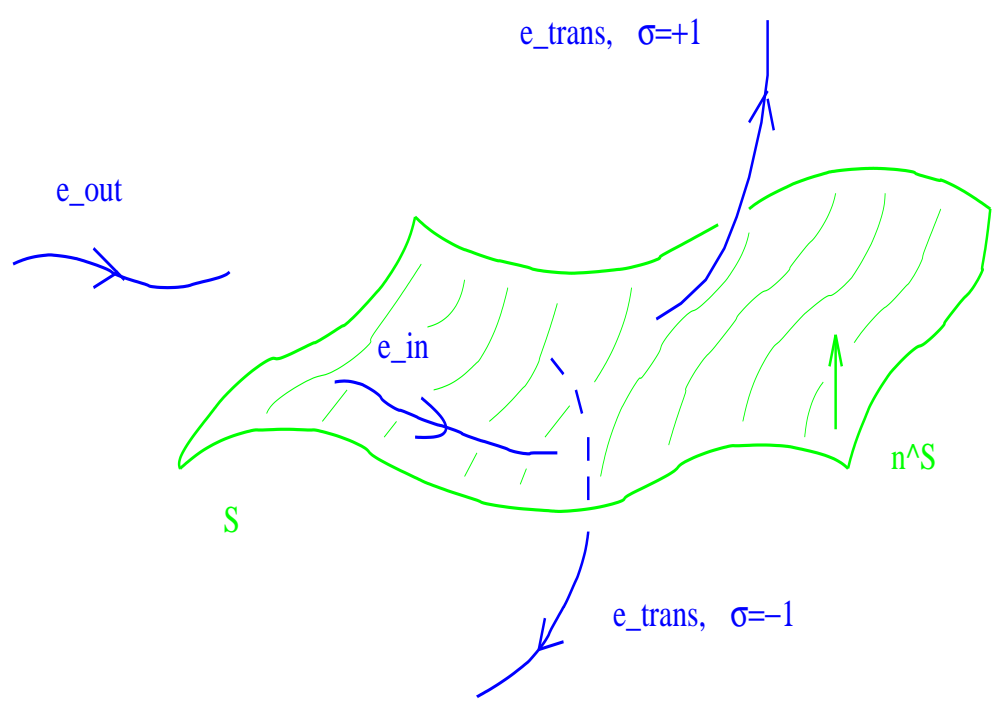

Figure 7: Intersection structure of surfaces with edges

orientation if necessary as depicted in figure 7 (strictly speaking, this is true only if $S$ is compactly supported, open, oriented and analytic). We also need to introduce the function $\sigma(S, e)$ which vanishes for $e \in E_{\text {in }}(\gamma) \cup E_{\text {out }}(\gamma)$ and which is \pm 1 for $e \in E_{\text {trans }}(\gamma)$ if the orientations of $S$ and $e$ agree or disagree respectively. The easiest case is $e \in E_{\text {trans }}(\gamma), \sigma(S, e)=1$. We find

$$
\left\{E_{j}(S), A(e)\right\}=\kappa \int_{D} d^{2} u n_{a}^{S}(u) \int_{0}^{1} d s \dot{e}^{a}(s) \delta\left(X_{S}(u), e(s)\right) A\left(e_{s}\right) \frac{\tau_{j}}{2} A\left(e_{s}\right)^{-1} A(e)
$$

where $e_{s}(t):=e(s t)$. Noticing that the support of the $\delta$-distribution is at $X_{S}(u)=e(0)$ which is an interior point of $S$ but a boundary point of $e$, a careful analysis reveals that (II.1.1.10) reduces to

$$
\left\{E_{j}(S), A(e)\right\}=\frac{\kappa}{4} \tau_{j} A(e)
$$

With this result, (ㅍ.1.1.9) can be written in the compact form

$$
\left\{E_{j}(S), f\right\}(A)=\frac{\kappa}{4} \sum_{e \in E(\gamma)} \sigma(S, e)\left[R_{e}^{j} f_{\gamma}\right]_{\mid h_{e^{\prime}}=A\left(e^{\prime}\right)}
$$

where we have defined the right invariant vector fields

$$
\left.\left(R_{e}^{j} f_{\gamma}\right)\left(\left\{h_{e^{\prime}}\right\}_{e^{\prime} \in E(\gamma)}\right\}\right):=\left(\frac{d}{d t}\right)_{t=0} f_{\gamma}\left(\left\{h_{e^{\prime}}\right\}_{e^{\prime} \neq e}, e^{t \tau_{j}} h_{e}\right)
$$

We can now define the vector fields $v_{S}^{j}$ on $\mathrm{Cyl}^{\infty}$ by $v_{S}^{j}[f]:=\left\{E_{j}(S), f\right\}$ and arrive at the Poisson *-algebra $\mathcal{B}$ generated by the $v_{S}^{j}, f \in \mathrm{Cyl}^{\infty}$ with involution defined by complex conjugation through the general formula $\left[(f, v),\left(f^{\prime}, v^{\prime}\right)\right]=\left(v\left[f^{\prime}\right]-v^{\prime}[f],\left[v, v^{\prime}\right]\right)$.

\section{Exercise II.1.3.}

Fill the gaps between (II.1.1.9) and (II.1.1.19.

Hint:

Use formula II.1.1.9 in order to derive (II.1.1.10), then expand $X_{S}(u)-e(t)$ around $u=u_{0}$ defined by $X_{S}\left(u_{0}\right)=e(0)$ and $t=0$ to linear order in $u-u_{0}$ and sufficiently high order in $t$ to arrive at (II.1.1.11). (Notice that e is only transversal, so $\dot{e}(0)$ may be tangential to $S$ in e(0)!) Verify that the end result coincides with (M1.1.1.19). 
So we see that we arrive at a well defined algebra $\mathcal{B}$ by smearing the momenta in two dimensions. Could we also smear them in three dimensions ? The answer is negative: Consider a one-parameter family of surfaces $t \mapsto S_{t}$ and define $E_{j}(\{S\}):=\int d t E_{j}\left(S_{t}\right)$. Then $f \mapsto\left\{E_{j}(S), f\right\}$ maps $f$ out of $\mathrm{Cyl}^{\infty}$ because it involves an integral over $t$ and thus depends on an uncountably infinite number of edges rather than a finite number. Thus this algebra would not be closed so that if we would like to stick with at least countably infinite graphs then we are forced to stick with two dimensional smearings of the electric fluxes !

\section{II.1.2 Representation Theory of $\mathcal{B}$ and Suitable Kinematical Represen- tations}

The representation Theory of $\mathcal{B}$ has been considered only rather recently [35] and the analysis is not yet complete. However, if one sticks to irreducible representations for which 1) the flux operators are well-defined and self-adjoint (in other words, the corresponding one parameter unitary groups are weakly continuous) and 2) the representation is spatially diffeomorphism invariant, then the unique solution to the representation problem is the representation which we describe in this section.

This representation is of the form $\mathcal{H}_{0}=L_{2}\left(\overline{\mathcal{A}}, d \mu_{0}\right)$ where $\overline{\mathcal{A}}$ is a certain distributional extension of $\mathcal{A}$ and $\mu_{0}$ is a probability measure thereon. The most elegant description of this Hilbert space uses the theory of $C^{*}$-algebras [36] but fortunately there is a purely geometric description available [37 which is easier to access for the beginner. In what follows we assume for simplicity that $\sigma$ is an oriented, connected, simply connected smooth manifold. One can show that each smooth manifold admits at least one analytic structure (i.e. the atlas of charts consists of real analytic maps) and we assume to have picked one once and for all.

\section{II.1.2.1 Curves, Paths, Graphs and Groupoids}

\section{Definition II.1.2.}

i)

By a curve $c$ we mean a map $c:[0,1] \rightarrow \sigma$ which is piecewise analytic, continuous, oriented and an embedding (does not come arbitrarily close to itself). It is automatically compactly supported. The set of curves is denoted $\mathcal{C}$ in what follows.

ii)

On $\mathcal{C}$ we define maps $\circ,(.)^{-1}$ called composition and inversion respectively by

$$
\left[c_{1} \circ c_{2}\right](t)=\left\{\begin{array}{cc}
c_{1}(2 t) & t \in\left[0, \frac{1}{2}\right] \\
c_{2}(2 t-1) & t \in\left[\frac{1}{2}, 1\right]
\end{array}\right.
$$

if $f\left(c_{1}\right)=b\left(c_{2}\right)$ and

$$
c^{-1}(t)=c(1-2 t)
$$

iii)

By a path $p$ we mean an equivalence class of curves $c$ which differ from each other by a finite number of reparameterizations and retracings, that is, $c \sim c^{\prime}$ if there either exists a map $t \mapsto f(t), \dot{f}(t)>0$ with $c=c^{\prime} \circ f$ or we may write $c, c^{\prime}$ as compositions of segments in the form $c=s_{1} \circ s_{2}, c^{\prime}=s_{1} \circ s_{3} \circ s_{3}^{-1} \circ s_{2}$ (and finite combinations of such moves). Notice that a curve induces its orientation and its end points on its corresponding path. The set of paths is denoted by $\mathcal{P}$.

iv)

By a graph $\gamma$ we mean a finite collection of elements of $\mathcal{P}$. We may break paths into pieces such that 

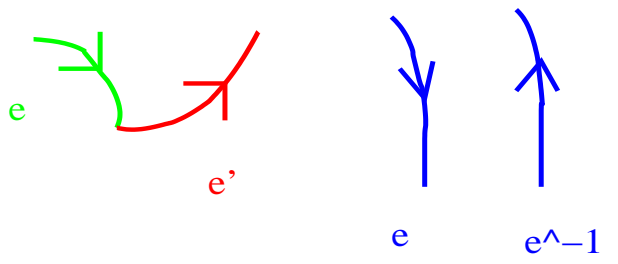

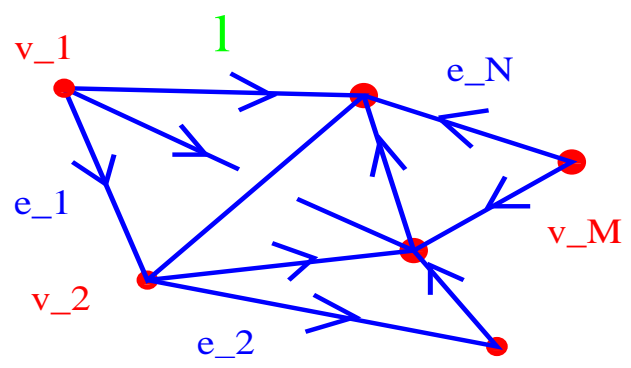

Figure 8: Paths and graphs

$\gamma$ can be thought of as a collection of edges $e \in E(\gamma)$, that is, paths which have an entire analytic representative and which intersect at most in their end points $v \in V(\gamma)$ called vertices. The set of graphs is denoted by $\Gamma$.

These objects are depicted in figure 8 .

\section{Exercise II.1.4.}

a)

Despite the name, composition and inversion does not equip $\mathcal{C}$ with a group structure for many reasons. Verify that composition is not associative and that the curve $c \circ c^{-1}$ is not simply b(c) but rather a retracing. Moreover, contemplate that $\mathcal{C}$ does not have a unit and that not every two elements can be composed.

b)

Define composition and inversion of paths by taking the equivalence class of the compositions and inversions of any of their representatives and check that this definition is well defined. Check that then composition of paths is associtive and that $p \circ p^{-1}=b(p)$. However, $\mathcal{P}$ still does not have a unit and still not every two elements can be composed.

c)

Let $O b j:=\sigma$ and for each $x, y \in \Sigma$ let $\operatorname{Mor}(x, y):=\{p \in \mathcal{P}: b(p)=x, f(p)=y\}$. Recall the mathematical definition of a category and conclude that $\mathcal{P}$ is a category in which every morphism is invertible, that is, a groupoid.

d)

Define the relation $\prec$ on $\Gamma$ by saying that $\gamma \prec \gamma^{\prime}$ if and only if every $e \in E(\gamma)$ is a finite composition of the $e^{\prime} \in E\left(\gamma^{\prime}\right)$ and their inverses. Verify that $\prec$ equips $\Gamma$ with the structure of a directed set, that is, for each $\gamma, \gamma^{\prime} \in \Gamma$ we find $\gamma^{\prime \prime} \in \Gamma$ such that $\gamma, \gamma^{\prime} \prec \gamma^{\prime \prime}$.

Hint:

For this to work, analyticity of the curve representatives is crucial. Smooth curves can intersect in Cantor sets and thus define graphs which are no longer finitely generated. Show first that this is not possible for analytic curves.

e)

Given a curve $c$ with path equivalence class $p$ notice that for the holonomy with respect to $A \in \mathcal{A}$ holds $A(c)=A(p)$. Contemplate that, in particular, every group is a groupoid and that every connection $A \in \mathcal{A}$ qualifies as a groupoid homomorphism, that is, $A: \mathcal{P} \rightarrow S U(2) ; p \mapsto A(p)$ with

$$
A\left(p \circ p^{\prime}\right)=A(p) A\left(p^{\prime}\right) \text { and } A\left(p^{-1}\right)=(A(p))^{-1}
$$

The fact that holonomies are really defined on paths rather than curves and that holonomies are characterized algebraically by $\llbracket 1.1 .2 .3$ makes the following definition rather natural. 
Definition II.1.3. The quantum configuration space is defined as the set $\overline{\mathcal{A}}:=\operatorname{Hom}(\mathcal{P}, S U(2))$ of all algebraic, arbitrarily non-continuous groupoid morphisms.

Here non-continuous means that in contrast to $A \in \mathcal{A}$ for an element $A \in \overline{\mathcal{A}}$ it is possible that $A(p)=1$ varies discontinuously as we vary $p$ continuously. Thus, $\overline{\mathcal{A}}$ can be thought of as a distributional extension of $\mathcal{A}$.

\section{II.1.2.2 Topology on $\overline{\mathcal{A}}$}

So far $\overline{\mathcal{A}}$ is just a set. We now equip it with a topology. The idea is actually quite simple. Recall the maps (II.1.1.8) which easily extend from $\mathcal{A}$ to $\overline{\mathcal{A}}$ and maps $\overline{\mathcal{A}}$ into $S U(2)^{|E(\gamma)|}$. Now $S U(2)^{|E(\gamma)|}$ is a compact Hausdorff topological group? in its natural manifold topology and we would like to exploit that. Thus we are motivated to consider the spaces $X_{\gamma}:=\operatorname{Hom}\left(\gamma, S U(2)^{|E(\gamma)|}\right)$ where $\gamma$ is considered as a subgroupoid of $\Gamma$ with objects $V(\gamma)$ and morphisms generated by the $e \in E(\gamma)$. The map

$$
X_{\gamma} \rightarrow S U(2)^{|E(\gamma)|} ; x_{\gamma} \mapsto\left\{x_{\gamma}(e)\right\}_{e \in E(\gamma)}
$$

identifies $X_{\gamma}$ with $G^{|E(\gamma)|}$ since $x_{\gamma} \in X_{\gamma}$ is already defined by which values it takes on the $e \in E(\gamma)$ and we may thus use this identification in order to equip $X_{\gamma}$ with a compact Hausdorff topology. Now consider the uncountably infinite product

$$
X_{\infty}:=\prod_{\gamma \in \Gamma} X_{\gamma}
$$

A standard result from general topology, Tychonov's theorem, tells us that the smallest topology on $X_{\infty}$ such that all the maps $p_{\gamma}: X_{\infty} \rightarrow X_{\gamma} ;\left(x_{\gamma}\right)_{\gamma \in \Gamma} \mapsto x_{\gamma}$ are continuous is a compact Hausdorff topology' Now we would like to identify $\overline{\mathcal{A}}$ with $X_{\infty}$ through the restriction map

$$
\Phi^{\prime}: \overline{\mathcal{A}} \rightarrow X_{\infty} ; A \mapsto\left(x_{\gamma}:=A_{\mid \gamma}=p_{\gamma}(A)\right)_{\gamma \in \Gamma}
$$

However, that map cannot be surjective because the points of $\overline{\mathcal{A}}$ satisfy the following constraint which encodes the algebraic properties of a generalized connection: Let $\gamma \prec \gamma^{\prime}$ and define the graph restriction maps

$$
p_{\gamma^{\prime} \gamma}: X_{\gamma^{\prime}} \rightarrow X_{\gamma} ; x_{\gamma^{\prime}} \mapsto\left(x_{\gamma^{\prime}}\right)_{\mid \gamma}
$$

which satisfy the compatibility condition

$$
p_{\gamma^{\prime \prime} \gamma}=p_{\gamma^{\prime} \gamma} \circ p_{\gamma^{\prime \prime} \gamma^{\prime}} \text { for } \gamma \prec \gamma^{\prime} \prec \gamma^{\prime \prime}
$$

Then automatically

$$
p_{\gamma^{\prime} \gamma}\left(A_{\mid \gamma^{\prime}}\right)=A_{\mid \gamma}
$$

We are therefore forced to consider the subset of $X_{\infty}$ defined by

$$
\bar{X}:=\left\{\left(x_{\gamma}\right)_{\gamma \in \Gamma} \in X_{\infty} ; p_{\gamma^{\prime} \gamma}\left(x_{\gamma}^{\prime}\right)=x_{\gamma} \forall \gamma \prec \gamma^{\prime}\right\}
$$

\footnotetext{
${ }^{5}$ Here it is crucial that $G=S U(2)$ is compact and thus for non-real Immirzi parameter all of what follows would be false.

${ }^{6}$ Recall that we know the topology on a space when we know a base of open sets from which we obtain all open sets by arbitrary unions and finite intersections. Since the preimages of open sets under continuous functions are open by definition, we obtain a topology once we know which functions are continuous.
} 


\section{Exercise II.1.5.}

i)

Show that the maps (II.1.2.7) are continuous surjections.

Hint:

Exploit the identification of the $X_{\gamma}$ with powers of $S U(2)$ and the continuity of multiplication and inversion in groups to establish continuity. To establish surjectivity use the fact that each edge e of $\gamma$ contains an edge $e_{e}^{\prime}$ of $\gamma^{\prime}$ as a segment such that the $e_{e}^{\prime}$ do not overlap each other. Now given $x_{\gamma}$ set $x_{\gamma^{\prime}}\left(e_{e}^{\prime}\right)=x_{\gamma}(e)$ and extend trivially away from the $e_{e}^{\prime}$. Check that this defines an element of $X_{\gamma^{\prime}}$. ii)

Show that $\bar{X}$ is a closed subset of $X_{\infty}$.

Hint:

Since $\bar{X}$ is not a metric space we must work with nets and show that every net of points $x^{\alpha} \in \bar{X}$ which converges in $X_{\infty}$ actually converges in $\bar{X}$. Using the defintion of the topology on $X_{\infty}$, show that this is equivalent to showing that the $p_{\gamma}\left(x^{\alpha}\right)=x_{\gamma}^{\alpha}$ converge to points $x_{\gamma}$ which satisfy (II.1.2.9) and verify this using continuity of the $p_{\gamma^{\prime} \gamma}$ just established.

The surjectivity of the $p_{\gamma^{\prime} \gamma}$ qualifies $\bar{X}$ as the so-called projective limit of the $X_{\gamma}$, a mathematical structure which is independent of our concrete context once we have a directed index set $\Gamma$ at our disposal and surjective projections which satisfy the compatibility condition ([I.1.2.8).

Now another standard result from topology now tells us that $\bar{X}$, being the closed subset of a compact Hausdorff space, is a compact Hausdorff space in the subspace topology and the question arises whether

$$
\Phi: \overline{\mathcal{A}} \rightarrow \bar{X} ; A \mapsto\left(x_{\gamma}:=A_{\mid \gamma}=p_{\gamma}(A)\right)_{\gamma \in \Gamma}
$$

is a bijection. Injectivity is fairly easy to see while surjectivity is a little bit tricky.

\section{Exercise II.1.6.}

Show that (II.1.2.11) is a bijection.

Hint:

Given $x \in \bar{X}$ and $p \in \mathcal{P}$ choose any $\gamma_{p} \in \Gamma$ such that $p \in \gamma_{p}$ and define $A_{x}$ by $A_{x}(p):=x_{\gamma_{p}}(p)$. Show that this definition is well defined using the directedness of $\Gamma$ and that $A_{x}$ is a groupoid homomorphism.

Let us collect these results in the following theorem [38].

\section{Theorem II.1.1.}

The space $\overline{\mathcal{A}}$ equipped with the weakest topology such that the maps $p_{\gamma}$ of (II.1.1.8) are continuous, is a compact Hausdorff space.

The value of this result is that it gives us a powerful tool for constructing measures on $\overline{\mathcal{A}}$.

\section{II.1.2.3 Measures on $\overline{\mathcal{A}}$}

A powerful theorem due to Riesz and Markov, sometimews called the Riesz representation theorem, tells us that there is a one - to - one correspondence between the positive linear functionals $\Lambda$ on the algebra $C(\overline{\mathcal{A}})$ of continuous functions on a compact Hausdorff space $\overline{\mathcal{A}}$ and (regular, Borel) probability measures $\mu$ thereon through the simple formula

$$
\Lambda(f):=\int_{\overline{\mathcal{A}}} d \mu(A) f(A)
$$


One says $\Lambda$ is represented by $f$. Here a linear functional is called positive if $\Lambda\left(|f|^{2}\right) \geq 0$ for any $f \in C(\overline{\mathcal{A}})$. A function algebra on a compact space can be equipped with the sup - norm $\|f\|:=\sup _{A \in \overline{\mathcal{A}}}|f(A)|$ which evidently has the so-called $C^{*}$-property $\|f \bar{f}\|=\|f\|^{2}$ so that (w.l.g. we may take $C(\overline{\mathcal{A}})$ to be complete w.r.t. the norm) $C(\overline{\mathcal{A}})$ is a $C^{*}$-algebra. A standard result in functional analysis reveals that positive linear functionals on $C^{*}$-algebras are automatically continuous, $|\Lambda(f)| \leq \Lambda(1)\|f\|$ and if we choose the normalization of $\Lambda$ to be $\Lambda(1)=1$ then $\mu$ is a probability measure.

In order to specify the measure $\mu_{0}$ that we are interested in, it is therefore enough to specify a positive linear functional $\Lambda_{0}$. The most elegant way of defining $\Lambda_{0}$ is through the following definition.

\section{Definition II.1.4.}

i)

Given a graph $\gamma$, label each edge $e \in E(\gamma)$ with a triple of numbers $\left(j_{e}, m_{e}, n_{e}\right)$ where $j_{e} \in\left\{\frac{1}{2}, 1, \frac{3}{2}, 2, ..\right\}$ is a half-integral spin quantum number and $m_{e}, n_{e} \in\left\{-j_{e},-j_{e}+1, \ldots, j_{e}\right\}$ are magnetic quantum numbers. A quadruple

$$
s:=\left(\gamma, \vec{j}:=\left\{j_{e}\right\}_{e \in E(\gamma)}, \vec{m}:=\left\{m_{e}\right\}_{e \in E(\gamma)}, \vec{n}:=\left\{n_{e}\right\}_{e \in E(\gamma)}\right)
$$

is called a spin network $(S N W)$. We also write $\gamma(s)$ etc. for the entries of a $S N W$.

ii)

Choose once and for all one representative $\rho_{j}, j>0$ half integral, from each equivalence class of irreducible representations of $S U(2)$. Then

$$
T_{s}: \overline{\mathcal{A}} \rightarrow \mathbb{C} ; A \mapsto \prod_{e \in E(\gamma)}\left[\sqrt{2 j_{e}+1}\left[\rho_{j_{e}}(A(e))\right]_{m_{e} n_{e}}\right]
$$

is called the spin-network function $(S N W F)$ of $s$. Here $\left[\rho_{j}(.)\right]_{m n}$ denotes the matrix elements of the matrix valued function $\rho_{j}($.$) .$

An example of a SNW, which can be arbitrarily large and with vertices of arbitrarily high valence, is given in figure 9. The original motivation for the definition of spin network functions 40] in loop quantum gravity was the fact that they are linearly independent in contrast to the Wilson loop functions which suffer from the so-called Mandelstam identities. For $S U(2)$ matrices $h, h^{\prime}$ they are $\operatorname{Tr}(h) \operatorname{Tr}\left(h^{\prime}\right)=\operatorname{Tr}\left(h h^{\prime}\right)+\operatorname{Tr}\left(h\left(h^{\prime}\right)^{-1}\right)$ and $\operatorname{Tr}(h)=\operatorname{Tr}\left(h^{-1}\right)$ which leads to an infinite tower of identities of the form

$$
\left[\operatorname{Tr}\left(A\left(\alpha_{1}\right)\right) \operatorname{Tr}\left(A\left(\alpha_{2}\right)\right)\right] \operatorname{Tr}\left(A\left(\alpha_{1}\right)\right)=\operatorname{Tr}\left(A\left(\alpha_{1}\right)\right)\left[\operatorname{Tr}\left(A\left(\alpha_{2}\right)\right) \operatorname{Tr}\left(A\left(\alpha_{1}\right)\right)\right]
$$

depending on how we bracket the product of traces involving the three loops $\alpha_{1}, \alpha_{2}, \alpha_{3}$ with a common base point. The SNWF's remove these cumbersome identities first by labelling functions by edges rather than loops and secondly by the simple observation that a tensor product of (fundamental) representations can be uniquely decomposed into irreducibles (Clebsh-Gordon decomposition).

\section{Theorem II.1.2.}

The uniform (Ashtekar - Lewandowski) measure $\mu_{0}$ is uniquely defined by the positive linear functional [39]

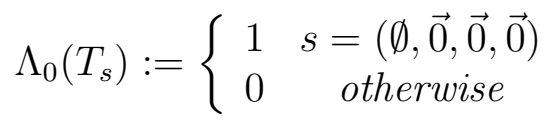




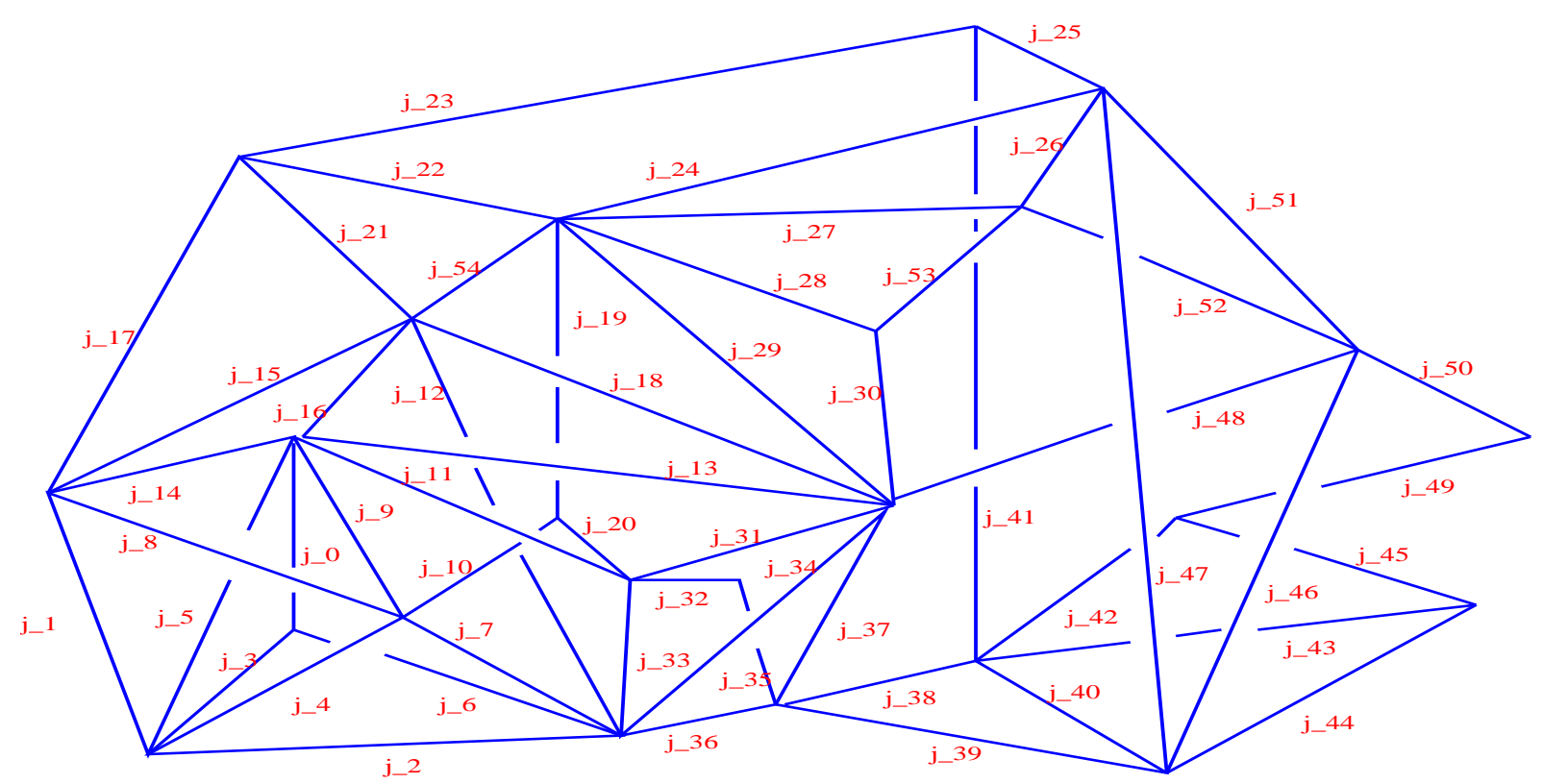

Figure 9: A SNW. Orientations and magnetic quantum numbers are suppressed

\section{Exercise II.1.7.}

i)

Recall the representation theory of $S U(2)$ from the quantum mechanics of angular momentum and verify that the SNWF are indeed linearly independent.

ii)

Verify that $\Lambda_{0}$ is a positive linear functional.

Hint:

Using the Stone - Weierstrass theorem, show first that the finite linear combinations of SNWF are dense in $C(\overline{\mathcal{A}})$. By continuity of $\Lambda_{0}$ it is therefore sufficient to check positivity on finite linear combinations

$$
f=\sum_{n=1}^{N} z_{n} T_{s_{n}}, \quad N<\infty, z_{n} \in \mathbb{C}
$$

with $s_{n}$ mutually different $S N W^{\prime} s$. To see this, verify that $\Lambda_{0}\left(\overline{T_{s}} T_{s^{\prime}}\right)=0$ for $s \neq s^{\prime}$ by using the Clebsh - Gordon formula $j \otimes j^{\prime} \equiv\left(j+j^{\prime}\right) \oplus\left(j+j^{\prime}-1\right) \oplus . . \oplus\left(\left|j-j^{\prime}\right|\right)$.

iii)

For the representation theory of compact groups fundamental is a theorem due to Peter and Weyl 41] which for $S U(2)$ amounts to saying that the functions

$$
T_{j m n}: S U(2) \rightarrow \mathbb{C} ; h \mapsto \sqrt{2 j+1}\left[\rho_{j}(h)\right]_{m n}
$$

form an orthonormal basis for the Hilbert space $L_{2}\left(S U(2), d \mu_{H}\right)$ where $\mu_{H}$ is the normalized Haar measure on $S U(2)$ (the unique normalized measure which invariant under inversion as well as left and right translation in $S U(2))$. Based on this result, show that the SNWF form an orthonormal basis for the Hilbert space $L_{2}\left(\overline{\mathcal{A}}, d \mu_{0}\right)$.

Let us summarize the results of the exercise in the following theorem [40.

\section{Theorem II.1.3.}

The kinematical Hilbert space $\mathcal{H}_{\text {kin }}:=L_{2}\left(\overline{\mathcal{A}}, d \mu_{0}\right)$ defined by (II.1.2.16) is non-separable and has the $S N W F$ 's $T_{s}$ as orthonormal basis. 


\section{II.1.2.4 Representation Property}

So far we did not verify that $\mathcal{H}_{k i n}$ is a representation space for our *-algebra $\mathcal{B}$ of basic operators. This will be done in the present section. Indeed, until today no other irreducible representation of the holonomy - flux algebra has been found (except if one allows also infinite graphs [42]).

By theorem (III.1.4) the subspace of finite linear combinations of SNWF's is dense in $\mathcal{H}_{k i n}$ with respect to the $L_{2}$ norm. On the other hand, we notice that the definition of $\mathrm{Cyl}^{\infty}(\mathcal{A})$ simply extends to $\mathrm{Cyl}^{\infty}(\overline{\mathcal{A}})$ and that finite linear combinations of SNWF's form a subspace of $\mathrm{Cyl}^{\infty}(\overline{\mathcal{A}})$. Thus, we may choose $\mathcal{D}_{\text {kin }}:=\operatorname{Cyl}^{\infty}(\overline{\mathcal{A}})$ and obtain a dense, invariant domain of $\mathcal{B}$ as we will see shortly. We define a representation of the holonomy - flux algebra by $\left(f^{\prime} \in \mathrm{Cy}^{\infty}(\mathcal{A}), f \in \mathrm{Cy}^{\infty}(\overline{\mathcal{A}}), A \in \overline{\mathcal{A}}\right)$

$$
\begin{aligned}
{\left[\pi(f) \cdot f^{\prime}\right](A) } & :=\left(f^{\prime} f\right)(A) \\
{\left[\pi\left(v_{S}^{j}\right) \cdot f\right](A) } & =\left[\pi\left(v_{S}^{j}\right) \pi(f) \cdot 1\right](A)=\left[\left(\left[\pi\left(v_{S}^{j}\right), \pi(f)\right]+\pi(f) \pi\left(E_{j}(S)\right)\right) \cdot 1\right](A) \\
& :=i \hbar\left[\pi\left(v_{S}^{j}[f]\right) \cdot 1\right](A)=i \hbar\left(v_{S}^{j}[f]\right)(A)
\end{aligned}
$$

Thus $\pi(f)$ is a multiplication operator while $\pi\left(v_{S}^{j}\right)$ is a true derivative operator, i.e. it annihilates constants. Notice that the canonical commutation relations are already obeyed by construction, thus we only need to verify the ${ }^{*}$-relations and the fact that $\pi\left(v_{S}^{j}\right)$ annihilates constants will be crucial for that.

The $\pi(f)$ are bounded multiplication operators (recall that smooth, i.e. in particular continuous, functions on copmpact spaces are uniformly bounded, that is, have a sup - norm) so that the adjoint is complex conjugation, therefore there is nothing to check. As for $\pi\left(v_{S}^{j}\right)$ we notice that given two smooth cylindrical functions on $\overline{\mathcal{A}}$ we always find a graph $\gamma$ over which both of them are cylindrical and which is already adapted to $S$.

\section{Exercise II.1.8.}

Let $f$ be cylindrical over $\gamma$. Verify that

$$
\Lambda_{0}(f)=\int_{S U(2)|E(\gamma)|} \prod_{e \in E(\gamma)} d \mu_{H}\left(h_{e}\right) f_{\gamma}\left(\left\{h_{e}\right\}_{e \in E(\gamma)}\right)
$$

Hint:

Write $f$ as a (Cauchy limit of) finite linear combinations of SNWF's and verify that (II.1.2.2Q) coincides with (11.1.2.10).

Using the explicit expression (II.1.1.12) and the result of exercise [1.1.8 it is easy to see that the symmetry condition $<f, \pi\left(v_{S}^{j}\right) f^{\prime}>_{k i n}=<\pi\left(v_{S}^{j}\right) f, f^{\prime}>_{k i n}$ is equivalent with the condition

$$
<F, R^{j} F^{\prime}>_{L_{2}\left(S U(2), d \mu_{H}\right)}=-<R^{j} F, F^{\prime}>_{L_{2}\left(S U(2), d \mu_{H}\right)}
$$

for any $F, F^{\prime} \in C^{\infty}(S U(2))$ and $R^{j}$ is the right invariant vector field on $S U(2)$. However, $\mu_{H}$ is by definition invariant under left translations and $R^{j}$ is a generator of left translations in $S U(2)$ so the result follows. This shows that $\mathcal{D}_{k i n}$ is contained in the domain of $\pi\left(v_{S}^{j}\right)^{\dagger}$ and that the restriction of the adjoint to $\mathcal{D}_{k i n}$ coincides with $\pi\left(v_{S}^{j}\right)$. That $\mathcal{D}_{k i n}$ is actually a domain of (essential) self-adjointness requires a little bit more work but is not difficult to see, e.g. [1].

Finally, let us verify that the representation is irreducible. By definition, a representation is irreducible if every vector is cyclic and a vector $\Omega$ is cyclic if the set of vectors $\pi(a) \Omega, a \in \mathcal{B}$ is dense. Now the vector $\Omega=1$ is cyclic because the vectors $\pi(f) \Omega=f, f \in \mathrm{Cyl}^{\infty}$ are already dense. Given an arbitrary element $\psi \in \mathcal{H}_{k i n}$ we know that it is a Cauchy limit of finite linear combinations of spin 
network functions. Thus, if we can show that we find a sequence $a_{n} \in \mathcal{B}$ such that $\pi\left(a_{n}\right) \psi$ converges to $\Omega$, then we are done. It is easy to see (exercise) that this problem is equivalent to showing that any $F \in L_{2}\left(F, d \mu_{H}\right)$ can be mapped by the algebra formed out of right invariant vector fields and smooth functions on $S U(2)$ to the constant function.

\section{Exercise II.1.9.}

Check that this is indeed the case.

Hint:

Show first that it is sufficient to establish that any polynomial $p$ of the $a, b, c, d, a d-b c=1$ for $h=\left(\begin{array}{ll}a & b \\ c & d\end{array}\right) \in S U(2)$ can be mapped to the constant function. Show then that suitable linear combinations of the $R^{j}, j=1,2,3$ with coefficients in $C^{\infty}(S U(2))$ produce the derivatives $\partial_{a}, \partial_{b}, \partial_{c}$ and convince yourself that $a^{N} p$ is a polynomial in $a, b, c$ for sufficiently large $N$.

Let us collect these results in the following theorem [43].

\section{Theorem II.1.4.}

The relations (II.1.2.19) define an irreducible representation of $\mathcal{B}$ on $\mathcal{H}_{\text {kin }}$.

Thus, the representation space $\mathcal{H}_{\text {kin }}$ constructed satisfies all the requirements that qualify it as a good kinematical starting point for solving the quantum constraints. Moreover, the measure $\mu_{0}$ is spatially diffeomorphism invariant as we will see shortly and together with the uniqueness result quoted at the beginning of this section, this is the only representation with that property. There are, however, doubts on physical grounds whether one should insist on a spatially diffeomorphism invariant representation because the smooth and even analytic structure of $\sigma$ which is encoded in the spatial diffeomorphism group should not play a fundamental role at short scales if Planck scale physics is fundamentally discrete. In fact, as we will see later, QGR predicts a discrete Planck scale structure and therefore the fact that we started with analytic data and ended up with discrete (discontinuous) spectra of operators looks awkward. Therefore, on the one hand we should keep in mind that other representations are possibly better suited in the final picture, on the other hand there is no logical contradiction within the present formulation and in fact in $2+1$ gravity one has a final combinatorical description while one started with analytical structures as well.

\section{II.2 Quantum Kinematics}

In this section we discuss the complete solution of the Gauss and Vector constraint as well as the quantization of kinematical, geometrical operators that measure the length, area and volume of coordinate curves, surfaces and regions respectively. We call these results kinematical because the Gauss and Vector constraint do not generate dynamics, this is the role of the Hamiltonian constraint which we will discuss in the third part. Moreover, the kinematical geometrical operators do not commute with the Vector constraint or the Hamiltonian constraint and are therefore not Dirac observables. However, as we will show, one can turn these operators easily into Dirac observables, at least with respect to the Vector constraint, and the fact that the spectrum is discrete is robust under those changes. 


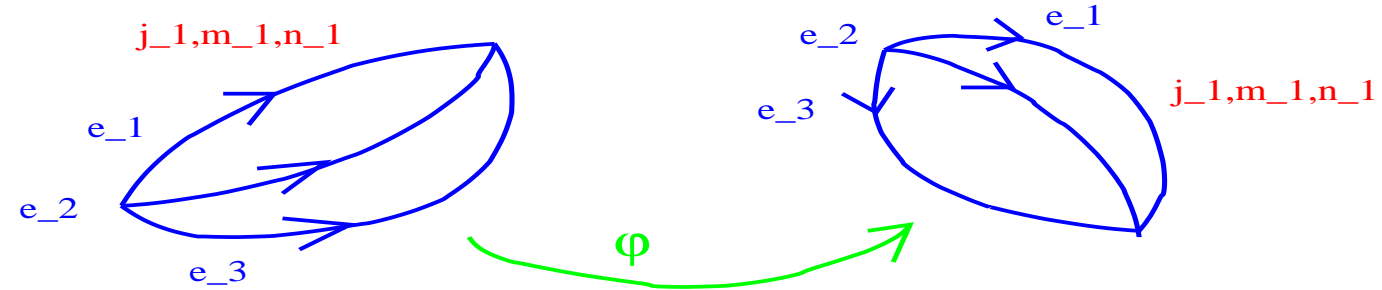

Figure 10: Action of Spatial Diffeomorphisms on SNW's

\section{II.2.1 The Space of Solutions to the Gauss and Spatial Diffeomorphism Constraint}

Recall the transformation behaviour of classical connections $A \in \mathcal{A}$ under $S U(2)$ gauge transformations and spatial diffeomorphisms $(\overline{\mathbb{I} .1 .1 .3})$. These equations trivially lift from $\mathcal{A}$ to $\overline{\mathcal{A}}$ and we may construct corresponding operators as follows: Let $\overline{\mathcal{G}}:=\operatorname{Fun}(\Sigma, S U(2))$ be the set of local gauge transformations without continuity requirement and consider the set Diff ${ }^{\omega}(\Sigma)$ of analytic diffeomorphisms. We are forced to consider analytic diffeomorphisms as otherwise we would destroy the analyticity of the elements of $\Gamma$. These two groups have a natural semi - direct product structure that has its origin in the algebra (I.2.2.7) and is given by

$$
\left[\overline{\mathcal{G}} \triangleright \operatorname{Diff}^{\omega}(\Sigma)\right] \times\left[\overline{\mathcal{G}} \triangleright \operatorname{Diff}^{\omega}(\Sigma)\right] \rightarrow\left[\overline{\mathcal{G}} \triangleright \operatorname{Diff}^{\omega}(\Sigma)\right] ; \quad[g, \varphi] \cdot\left[g^{\prime}, \varphi^{\prime}\right]=\left[\left(g^{\prime} \circ \varphi^{-1}\right) g, \varphi \circ \varphi^{\prime}\right]
$$

\section{Exercise II.2.1.}

Verify (II.2.1.1).

Hint:

Define $[g, i d] \cdot A:=A^{g},[i d, \varphi] \cdot A:=A^{\varphi}$ and $[g, \varphi] \cdot A:=[g, i d] \cdot([i d, \varphi] \cdot A)$.

We now define representations

$$
\begin{aligned}
& \hat{U}: \overline{\mathcal{G}} \rightarrow \mathcal{B}\left(\mathcal{H}_{k i n}\right) ; g \mapsto \hat{U}(g) \\
& \hat{V}: \operatorname{Diff}^{\omega}(\Sigma) \rightarrow \mathcal{B}\left(\mathcal{H}_{\text {kin }}\right) ; \varphi \mapsto \hat{V}(\varphi)
\end{aligned}
$$

densely on $f=p_{\gamma}^{*} f_{\gamma} \in \mathcal{D}_{\text {kin }}$ by

$$
\begin{aligned}
{[\hat{U}(g) f](A) } & \left.:=f_{\gamma}\left(\left\{g(b(e)) A(e) g(f(e))^{-1}\right\}_{e \in E(\gamma)}\right\}\right) \\
{[\hat{V}(\varphi) f](A) } & \left.:=f_{\gamma}\left(\left\{A\left(\varphi^{-1}(e)\right)\right\}_{e \in E(\gamma)}\right\}\right)
\end{aligned}
$$

Here $\mathcal{B}($.$) denotes the bounded operators on a Hilbert space. This definition of course comes precisely$ from the classical formula ([1.1.1.3). The action of a diffeomorphism on a SNWF $T_{s}$ is therefore simply by mapping the graph $\gamma(s)$ to $\varphi^{-1}(s)$ while the labels $j_{e}, m_{e}, n_{e}$ are carried from $e$ to $\varphi^{-1}(e)$ as depicted in figure 10. Then the following theorem holds 43 .

\section{Theorem II.2.1.}

The relations (II.2.1.3) define a unitary representation of the semi - direct product kinematical group $\overline{\mathcal{G}} \times \operatorname{Diff}^{\omega}(\Sigma)$.

\section{Exercise II.2.2.}

Prove theorem (II.2.1).

Hint:

Check unitarity on the SNWF basis using the bi - invariance of the Haar measure. That (II.2.1.3) holds can be traced back to exercise [1.2.1. 
The unitarity of the kinematical gauge group implies invariance of the measure $\mu_{0}$ and thus supplies additional motivation for the representation space $\mathcal{H}_{\text {kin }}$. Notice that the statement that (II.2.1.3) defines a representation in particular means that the kinematical constraint algebra is free of anomalies. This should be contrasted with string theory where the anomly sits also in the spatial diffeomorphism group (e.g. $\operatorname{Diff}\left(S^{1}\right)$ for the closed string) unless one chooses the critical dimension $D=25(9)$ for the bosonic (supersymmetric) string.

Let us now solve the kinematical constraints. By definition, we are supposed to find algebraic distributions $l \in \mathcal{D}_{k i n}^{*}$ which satisfy

$$
l(\hat{U}(g) f)=l(\hat{V}(\varphi) f)=l(f) \forall g \in \overline{\mathcal{G}}, \varphi \in \operatorname{Diff}^{\omega}(\Sigma), f \in \mathcal{D}_{k i n}
$$

Now it is not difficult to see that any element of $\mathcal{D}_{k i n}^{*}$ can be conveniently written in the form

$$
l(.)=\sum_{s} c_{s}<T_{s}, .>_{k i n}
$$

where $c_{s}$ are complex valued coefficients and the uncountably infinite sum extends over all possible SNW's. The general solution to ([I.2.1.4) is then easy to describe: Invariance under $\overline{\mathcal{G}}$ means that for fixed $\gamma$ the coefficients $c_{\gamma, \vec{j}, \vec{m}, \vec{n}}$ have to be chosen, as $\vec{j}, \vec{m}, \vec{n}$ vary, in such a way that at each vertex of $\gamma$ the resulting function is gauge invariant. That is, if $j_{1}, . ., j_{n}$ are the labels of edges incident at $v$, then the $c_{s}$ have to arrange themselves to a projector on the trivial representations contained in the tensor product $j_{1} \otimes . . \otimes j_{n}$. Such a projector is also called intertwiner in the mathematical literature. For $S U(2)$ this leads to the theory of Clebsh-Gordon coefficients, $6 j$-symbols etc. As for Diff ${ }^{\omega}(\Sigma)$ we see that $c_{\varphi(\gamma), \vec{j}, \vec{m}, \vec{n}}$ must be independent of $\varphi$, therefore $c_{\varphi, \vec{j}, \vec{m}, \vec{n}}$ depends only on the generalized knot class of $\gamma$ ! We say generalized because, as we will see later on, the physically relevant graphs are those with self-intersections while classical knot theory deals only with smooth curves.

One may ask whether one should already define a physical inner product with respect to the Gauss and spatial Diffeomorphism constraint and then solve the Hamiltonian constraint in a second, separate step on that already partly physical Hilbert space. While such a Hilbert space can indeed be constructed [43] it is of no use for QGR because the Hamiltonian constraint cannot leave that Hilbert space invariant as we see from the second equation in (I.2.1.12) and we must construct the physical inner product from the full solution space to all constraints. However, at least with respect to the kinematical constraints the full quantization programme including the question of observables has already been completed except for the analysis of the classical limit.

\section{II.2.2 Kinematical Geometrical Operators}

We will restrict ourselves to the description of the area operator the classical expression of which we already wrote in ([I.1.1.6) and (II.1.1.7).

In order to quantize $\operatorname{Ar}(S)$ one starts from (II.1.1.7) and decomposes the analytical, compactly supported and oriented surface $S$ or, equivalently, its preimage $D$ under $X_{S}$ into small pieces $S_{I}$. Then the exact area functional is approximated by the Riemann sum

$$
\operatorname{Ar}(\{S\})=\beta \sum_{I} \sqrt{E_{j}\left(S_{I}\right)^{2}}
$$

This function is easily quantized because $\hat{E}_{j}\left(S_{I}\right)=i \hbar v_{s}^{j}$ is a self-adjoint operator so that the sum over $j$ of its squares is positive semi-definite, hence its square root is well-defined. Let us denote the 
resulting, partition dependent operator by $\widehat{\operatorname{Ar}}(\{S\})$. Now one can show that the (strong) limit as

the partition is sent to the continuum exists [44] and a partition independent operator $\widehat{\operatorname{Ar}}(S)$ results [44.

\section{Theorem II.2.2.}

The area functional admits a well-defined quantization $\widehat{\operatorname{Ar}}(S)$ on $\mathcal{H}_{\text {kin }}$ with the following properties:

i) $\widehat{A r}(S)$ is positive semidefinite, (essentially) self-adjoint with $\operatorname{Cy}^{2}(\overline{\mathcal{A}})$ as domain of (essential) self-adjointness.

ii) The spectrum Spec $(\widehat{A r}(S))$ is pure point (discrete) with eigenvectors being given by finite linear combinations of spin network functions.

iii) The eigenvalues are given explicitly by

$$
\begin{aligned}
\lambda_{j_{1}, j_{2}, j_{12}} & =\frac{\beta \ell_{P}^{2}}{4} \sqrt{2 j_{1}\left(j_{1}+1\right)+2 j_{2}\left(j_{2}+1\right)-j_{12}\left(j_{12}+1\right.} \\
J_{12} & \in\left\{j_{1}+j_{2}, j_{1}+j_{2}-1, . .,\left|j_{1}-j_{2}\right|\right\}
\end{aligned}
$$

where $j_{1}, j_{2}$ are spin quantum numbers and $\ell_{P}^{2}=\hbar \kappa$ is the Planck area. The spectrum has an area gap (smallest non-vanishing eigenvalue) given by

$$
\lambda_{0}=\beta \ell_{P}^{2} \frac{\sqrt{3}}{4}
$$

iv) Spec $(\widehat{A r}(S))$ contains information about the topology of $S$, for instance it matters whether $\partial S=\emptyset$ or not.

\section{Exercise II.2.3.}

Verify that the area gap is indeed given by (II.2.2.3) and check that the distance between subsequent eigenvalues rapidly decreases as $j_{1}, j_{2} \rightarrow \infty$. Can one give an asymptotic formula for $N(A)$, the number of eigenvalues (discarding multiplicity) in the interval $\left[A-\ell_{P}^{2}, A+\ell_{P}^{2}\right]$ ? Thus, a correspondence principle, important for the classical limit is valid. If the spectrum would only consist of the main series $\lambda_{j}=\frac{\ell_{P}^{2}}{2} \sqrt{j(j+1)}$ which one obtains for $j_{1}=j_{2}=j, j_{12}=0$ then such a correspondence principle would certainly not hold which is, e.g., relevant for the black body spectrum of the Hawking radiation.

A)

Theorem $\amalg 1.2 .2$ is an amazing result for several reasons:

First of all, the expression for $\operatorname{Ar}(S)$ depends non-polynomially, not even analytically on the product $E_{j}^{a}(x) E_{j}^{b}(x), x \in S$. Now $E_{j}^{a}(x)$ becomes an operator valued distribution in the quantum theory and products of distributions at the same point are usually badly divergent. However, $\widehat{\operatorname{Ar}}(S)$ is perfectly well-defined! This is the first pay-off for sticking to a rigorous and background independent formalism !

B)

Although $S, \gamma, \Sigma, .$. are analytical, the spectrum $\operatorname{Spec}(\widehat{\operatorname{Ar}}(S))$ is discrete. In other words, suppose we are measuring the area of a sheet of paper with a spin-network state. As long as the sheet does not cut an edge of the graph, the area eigenvalue is exactly zero no matter how "close" the edge and the sheet are. We have put the word "close" in inverted commas because this word has no meaning: 
Since there is no background metric, we do not know what close means, only diffeomorphism invariant notions have a meaning such as "the edge is cut" or "the edge is not cut". However, once the edge is cut the area eigenvalue jumps at least by the area gap. This strongly hints that the microscopical geometry is really distributional (discontinuous) and that we have a discrete Planck scale structure, the role of the atoms of geometry being played by the one-dimensional (polymer-like) excitations labelled by SNW's. One may speculate that this discrete structure is fundamental and that the analyticity assumptions that we began with should be unimportant, in the final picture everything should be only combinatorical. The smooth geometry that we are familiar with at macroscopic scales is merely a result of coarse graining, for instance in order that a SNWF labelled with spin $j=1 / 2$ on every edge assigns to our sheet of paper its area of about $100 \mathrm{~cm}^{2}$, an order of $10^{68}$ edges of the SNW have to cut the sheet!

C)

Qualitatively similar results apply to the volume operator $\widehat{\operatorname{Vol}}(R)$ 44, 45 and the length operator $\widehat{\operatorname{Len}}(c)$ 46 whose classical expressions are given by

$$
\operatorname{Vol}(R)=\int_{R} d^{3} x \sqrt{\operatorname{det}(q)} \text { and } \operatorname{Len}(c)=\int_{c} \sqrt{q_{a b} d x^{a} d x^{b}}
$$

D)

These kinematical operators are certainly not Dirac observables because they are not even spatially diffeomorphism invariant (but $S U(2)$ invariant) since the objects $R, S, c$ are just coordinate submanifolds of $\Sigma$. Thus, one may wonder whether the properties of the spectrum just stated have any significance at all. The answer is believed to be affirmative as the following argument shows: For instance, instead of $\mathrm{Vol}(R)$ consider

$$
\operatorname{Vol}_{E M}=\int_{\Sigma} d^{3} x \sqrt{\operatorname{det}(q)} \theta\left(\frac{q_{a b}}{\sqrt{\operatorname{det}(q)}}\left[E^{a} E^{b}+B^{a} B^{b}\right]\right)
$$

where we have coupled a Maxwell field to GR with electromagnetic fields $E^{a}, E^{b}$ and $\theta$ is the step function. The physical meaning of (II.2.2.5) is that it measures the volume of the region where the electromagnetic field energy density is non-vanishing and it is easy to check that (III.2.2.5) is actually spatially diffeomorphism invariant! Now in QGR the argument of the step function can be given a meaning as an operator (valued distribution) as we will see in the next section and the theta function of an operator can be defined through the spectral theorem. Since the spectrum of the $\theta$-function consists only of $\{0,1\}$, the spectrum of ([1.2.2.5) should actually coincide with that of $\widehat{\operatorname{Vol}}(R)$ 47]. A similar argument should also be valid with respect to Dirac observables commuting with the Hamiltonian constraint.

E)

The existence of the area gap is also at the heart of the finiteness of the Bekenstein - Hawking entropy of black holes as we will see. 


\section{Part III}

\section{Selected Areas of Current Research}




\section{III.1 Quantum Dynamics}

The Hamiltonian constraint $C$ of QGR is, arguably, the holy grail of this approach to quantum gravity, therefore we will devote a substantial amount of space to this subject. In fact, unless one can quantize the Hamiltonian constraint, literally no further progress can be made so that it is important to know what its status is. From the explicit, non-polynomial expression (I.2.2.5) it is clear that a welldefined operator version of this object will be extremely hard to obtain and in fact this had been the major obstacle in the whole approach until the mid 90's. In particular, within the original ADM formulation only formal results were available. However, since with the new connection formulation also the non-polynomial kinematical operators of the previous section could be constructed, chances might be better.

At this point we include a brief account of the historical development of the subject in order to avoid confusion as one looks at older papers:

Originally one chose the Immirzi parameter as $\beta= \pm i$ and considered $\tilde{C}=\sqrt{\operatorname{det}(q)}$ rather than $C$ because then $\tilde{C}$ is actually a simple polynomial of only fourth order (the "More" term disappears). Polynomiality was considered as mandatory. There were three problems with this idea:

1) The non-polynomiality was shifted from $C$ into the reality conditions $A+\bar{A}=2 \Gamma(E)$ where the spin connection $\Gamma$ is now a highly non-polynomial function of $E$. The operator version of this equation should be very hard to implement.

2) If $A$ is complex, then we are dealing with an $S L(2, \mathbb{C})$ bundle rather than an $S U(2)$ bundle. Since $S L(2, \mathbb{C})$ is not compact, the mathematical apparatus of section $\mathbb{I}$ is blown away.

3) Even formal trials to quantize $\tilde{C}$ resulted in either divergent, or background dependent operators. In [27] it was suggested to keep $\beta$ real which solves problems 1) and 2), however, then $\tilde{C}$ becomes even more complicated and anyway problem 3) is not cured. Finally in [48] it was shown that nonpolynomiality is not necessarily an obstacle, even better, it is actually required in order to arrive at a well-defined operator: It was established that the reason for problem 3) is that $\tilde{C}$ is a scalar density of weight two while it was shown that only density weight one scalars have a chance to be quantized rigorously and background independently. Therefore the currently accepted point of view is that $\beta$ should be real and that one uses the original unrescaled $C$ rather than $\tilde{C}$.

\section{III.1.1 A Possible New Mechanism for Avoiding UV Singularities in Background Independent Quantum Field Theories}

Before we go into more details concerning [48], let us give a heuristic explanation just why it happens that $Q G R$ may cure $U V$ problems of QFT, making the connection with the issue of the density weight just mentioned. Consider classical Einstein - Maxwell theory on $M=\mathbb{R} \times \sigma$ in its canonical formulation, then the Hamiltonian constraint gains an extra matter piece given for unit lapse $N=1$ by

$$
H_{E M}=\frac{1}{2 e^{2}} \int_{\sigma} d^{3} x \overbrace{\frac{q_{a b}}{\sqrt{\operatorname{det}(q)}}}^{\text {Density weight }-1} \underbrace{\left[E^{a} E^{b}+B^{a} B^{b}\right]}_{\text {Density weight }+2}
$$

Exercise III.1.1.

Starting from the Lagrangean

$$
L=-\frac{1}{4 e^{2}} \sqrt{|\operatorname{det}(g)|} F_{\mu \nu} F_{\rho \sigma} g^{\mu \rho} g^{\nu \sigma}
$$


where $F=2 d A$ is the spacetime curvature of the Maxwell connection A with unit $\mathrm{cm}^{-1}$ and $e$ is the electric charge in units such that $\alpha=\hbar e^{2}$ is the dimensionfree Feinstrukturkonstante, perform the Legendre transform. With the electric field $E^{a}$ being the momentum conjugate to the spatial piece $A_{a}$ of A verify that the "Hamiltonian" is given by $-A_{0} G+N^{a} V_{a}^{\prime}+N C^{\prime}$ where $G=\partial_{a} E^{a}$ is the Gauss law, $V_{a}^{\prime}=F_{a b} E^{b}$ and $C^{\prime}$ is the integrand of (III.1.1.1) with $B^{a}=\epsilon^{a b c} F_{b c} / 2$ the magnetic field. Check that $G^{\prime}$ generates $U(1)$ gauge transformations while $V_{a}^{\prime}$ generates spatial diffeomorphisms where $A_{a}, E^{a}$ transform as a one - form and a vector density of weight one respectively. Conirm that also $B^{a}$ is a vector density of weight one.

As the exercise reveals, the geometry factor in (III.1.1.1) is a symmetric covariant tensor of rank two of density weight -1 due to the factor $\sqrt{\operatorname{det}(q)}$ in the denominator while the matter part is a symmetric contravariant tensor of rank two of density weight +2 . That the resulting scalar has net density weight is +1 is no coincidence but a direct consequence of the diffeomorphism invariance or background independence of any matter theory coupled to gravity: only the integral over $\sigma$ of a scalar density of weight +1 is spatially diffeomorphism invariant.

We can now quantize (III.1.1.1) in two ways:

1)

In the first version we notice that if $g=\eta$ is the Minkowski metric, that is, $q_{a b}=\delta_{a b}$ then ([II.1.1.1) reduces to the ordinary Maxwell Hamiltonian on Minkowski space. Thus we apply the formalism of QFT on a background spacetime, in this case Minkowski space, because we have fixed $q_{a b}$ to the non-dynamical $\mathbb{C}$-number field $\delta_{a b}$ which is not quantized at all.

2)

In the second version we keep $q_{a b}$ dynamical and quantize it as well. Thus we apply QGR, a background independent quantization. Now $q_{a b}$ becomes a field operator $\hat{q}_{a b}$ and the statement that the metric is flat can at most have a semiclassical meaning, that is, the expectation value of $\hat{q}_{a b}$ in a gravitational state is close to $\delta_{a b}$.

Let us now sketch how these two different quantizations are performed and exactly pin-point how it happens that the first quantization is divergent while the second is finite.

1) QFT on a background spacetime

As we have said, the metric $q_{a b}=\delta_{a b}$ is now no longer a dynamical entitity but just becomes a complex number. What we get is the usual Maxwell Hamiltonian operator

$$
\hat{H}_{M}=\frac{1}{2 e^{2}} \int_{\Sigma} d^{3} x \delta_{a b}\left[\hat{E}^{a} \hat{E}^{b}+\hat{B}^{a} \hat{B}^{b}\right]
$$

Notice the crucial difference with ([II.1.1.1): The net density weight of the operator valued distribution in the integral is now +2 rather than +1 ! By switching off the metric as a dynamical field we have done a severe crime to the operator, because the net density weight +2 will be remembered by the operator in any faithful representation of the canonical commutation relations and leads to the following problem: The only coordinate density of weight one that one can construct is a $\delta$-distribution (and derivatives thereof), thus for instance the operator $\hat{E}^{a}(x)$ is usually represented as a functional derivative which one can rewrite formally as

$$
\alpha \delta / \delta A_{a}(x)=\alpha \sum_{y \in \Sigma} \delta(x, y) \partial / \partial A_{a}(y)
$$

The right hand side of ([II.1.1.4) is a sum over terms each of which consists of a well-defined operator $Y_{a}(y)=\partial / \partial A_{a}(y)$ and a distributional prefactor $\delta(x, y)$. It is for this reason that 
expressions of the form $\hat{E}^{a}(x) \hat{E}^{b}(x)$ cannot be well-defined since we get products of distributions supported at the same point $x$ and which result in divergent expressions of the form $\sum_{y, z} \delta(x, y) \delta(x, z) Y_{a}(y) Y_{b}(z)=\sum_{y} \delta(x, y)^{2} Y_{a}(y) Y_{b}(y)$. The density weight two is correctly encoded in the term $\delta(x, y)^{2}=\delta(0,0) \delta(x, y)$ which, however, is meaningless.

These heuristic arguments can of course be made precise: (III.1.1.3) is quantized on the Fock space $\mathcal{H}_{\text {Fock }}$ and one obtains

$$
\hat{H}_{M}=: \hat{H}_{M}:+\hbar \int_{\Sigma} \underbrace{\left[\sqrt{-\Delta_{x}} \delta(x, y)\right]_{x=y}}_{\text {UV Singularity }}
$$

Here the colons stand for normal ordering. The UV (or short distance) singularity is explicitly identified as the coincidence limit $x=y$ of the integrand in the normal ordering correction. Therefore $\hat{H}_{M}$ is ill-defined on $\mathcal{H}_{\text {Fock }}$. Notice that even if the integrand would be finite, the integral suffers from an IR (or large volume) singularity if $\sigma$ is not compact which comes from the fact that we are dealing with an infinite number of degrees of freedom. This singularity is, in contrast to the UV singularity, physical since it captures the vacuum energy of the universe which is of course infinite if the volume is.

2) QFT coupled to $Q G R$

This time we keep the metric as a dynamical variable and quantize it. Thus instead of ([II.1.1.3) we obtain something of the form

$$
\hat{H}_{E M}=\frac{1}{2 e^{2}} \int_{\Sigma} d^{3} x \frac{\widehat{q_{a b}}}{\sqrt{\operatorname{det}(q)}}\left[\hat{E}^{a} \hat{E}^{b}+\hat{B}^{a} \hat{B}^{b}\right]
$$

This time the net density weight is still +1 . Now while the expression ([II.1.1.4) is still valid and implies that there will be a product of $\delta$-distributions in the numerator coming from the matter operator valued distributions, there is also a $\delta$-distribution in the denominator due to the factor $\sqrt{\operatorname{det}(q)}$ which comes about as follows: As we already mentioned in section 【I.2.2 the volume functional in ([1.2.2.4) admits a well-defined quantization of the form

$$
\widehat{\operatorname{Vol}}(R) T_{s}=\ell_{P}^{3} \sum_{v \in V(\gamma(s)) \cap R} \hat{V}_{v} T_{s}
$$

where $\hat{V}_{v}$ is a well-defined, dimensionfree operator (not an operator valued distribution !) built from the vector fields $v_{S}^{j}$. Since $\operatorname{Vol}(R)$ is the integral over $R$ of $\sqrt{\operatorname{det}(q)}$ we conclude that $\sqrt{\operatorname{det}(q)}$ admits a quantization as an operator valued distribution, namely

$$
\widehat{\sqrt{\operatorname{det}(q)}}(x) T_{s}=\ell_{P}^{3} \sum_{v \in V(\gamma(s))} \delta(x, v) \hat{V}_{v} T_{s}
$$

Now certainly (III.1.1.6) cannot be quantized on the Hilbert space $\mathcal{H}_{\text {kin }} \otimes \mathcal{H}_{\text {Fock }}$ because $\mathcal{H}_{\text {Fock }}$ depends on a background metric (for instance through the Laplacian $\Delta$ ) which is not available to us. However, we may construct a background independent Hilbert space $\mathcal{H}_{\text {kin }}^{\prime}$ for Maxwell theory which is completely identical to our $\mathcal{H}_{k i n}$, just that $S U(2)$ is replaced by $U(1)$ 48]. In $\mathcal{H}_{k i n}^{\prime}$ the role of spin network states is played by charge network (CNW) states, that is, edges $e$ are labelled by integers $n_{e}$ (irreducibles of $U(1)$. Let us denote CNW's by $c=(\gamma, \vec{n}=$ 
$\left.\left\{n_{e}\right\}_{e \in E(\gamma)}\right)$ and CNWF's by $T_{c}^{\prime}$. Then a basis for the Einstein-Maxwell theory kinematical Hilbert space $\mathcal{H}_{\text {kin }} \times \mathcal{H}_{\text {kin }}^{\prime}$ is given by the states $T_{s} \otimes T_{c}^{\prime}$.

Now something very beautiful happens, which is not put in by hand but rather is a derived result: A priori the states $T_{s}, T_{c}^{\prime}$ may live on different graphs, however, unless the graphs are identical, the operator automatically ([II.1.1.6) annihilates $T_{s} \otimes T_{c}^{\prime}$ [49]. This is the mathematical manifestation of the following deep physical statement: Matter can only exist where geometry is excited. Indeed, if we have a gravitational state which has no excitations in a coordinate region $R$ then the volume of that region as measured by the volume operator is identically zero. However, if a coordinate region has zero volume, then it is physically simply not there, it is empty space. Summarizing, the operator (III.1.1.6) is non-trivial only if $\gamma(s)=\gamma(c)$.

With this being understood, let us then sketch the action of ([II.1.1.6) on our basis. One finds heuristically

$$
\begin{gathered}
\hat{H}_{E M} T_{s} \otimes T_{c}^{\prime}=m_{P} \sum_{v \in V(\gamma)} \sum_{e, e^{\prime} \in E(\gamma), e \cap e^{\prime}=v} \times \\
\times \int_{\Sigma} d^{3} x[\frac{\hat{q}_{e, e^{\prime}}}{\hat{V}_{v}} \underbrace{\underbrace{\frac{1}{\delta(x, v)}}_{\uparrow} T_{s}] \otimes[\underbrace{\delta(x, v)}_{\uparrow} \delta(y, v) Y^{e} Y^{e^{\prime}} T_{c}^{\prime}]_{x=y}]}_{\text {Cancellation }}
\end{gathered}
$$

where $m_{P}=\sqrt{\hbar / \kappa}$ is the Planck mass. Here $\hat{q}_{e, e^{\prime}}$ and $Y^{e}$ are well-defined, dimensionfree operators (not distribution valued !) on $\mathcal{H}_{\text {kin }}$ and $\mathcal{H}_{k i n}^{\prime}$ respectively built from the right invariant vector fields $R_{e}^{j}, R_{e}$ that enter the definition of the flux operators as in ([1.1.1.12) and its analog for $U(1)$. The product of $\delta$-distributions in the numerator of (III.1.1.9) has its origin again in the fact that the matter operator has density weight +2 certainly also in this representation and therefore has to be there, so nothing is swept under the rug! The $\delta$-distribution in the denominator comes from ([II.1.1.8) and correctly accounts for the fact that the geometry operator has density weight -1 . Again we have a coincidence limit $x=y$ which comes from a point splitting regularization and which in the background dependent quantization gave rise to the UV singularity. Now we see what happens: One of the $\delta$-distributions in the numerator gets precisely cancelled by the one in the denominator leaving us with only one $\delta$-distribution correctly accounting for the fact that the net density weight is +1 . The integrand is then well-defined and the integral can be performed resulting in the finite expression

$$
\begin{gathered}
\hat{H}_{E M} T_{s} \otimes T_{c}^{\prime}=\sum_{v \in V(\gamma)} \sum_{e, e^{\prime} \in E(\gamma), e \cap e^{\prime}=v} \times \\
\times\left[\frac{\hat{q}_{e, e^{\prime}}}{\hat{V}_{v}} T_{s}\right] \otimes\left[Y^{e} Y^{e^{\prime}} T_{c}^{\prime}\right]_{x=y}
\end{gathered}
$$

Notice that finite here means non-perturbatively finite, that is, not only finite order by order in perturbation theory (notice that in coupling gravity we have a highly interacting theory in front of us). Thus, comparing our non-perturbative result to perturbation theory the result obtained is comparable to showing that the perturbation series converges! Notice also that for non-compact $\sigma$ the expression (III.1.1.10) possibly has the physically correct IR divergence coming from a sum over an infinite number of vertices. 


\section{Exercise III.1.2.}

Recall the Fock space quantization of the Maxwell field and verify (III.1.1.5).

This ends our heuristic discussion about the origin of UV finiteness in QGR. The crucial point is obviously the density weight of the operator in question which should be precisely +1 in order to arrive at a well-defined, background independent result: Higher density weight obviously leads to more and more divergent expressions, lower density weight ends in zero operators.

\section{III.1.2 Sketch of a Possible Quantization of the Hamiltonian Constraint}

We now understand intuitively why the rescaled Hamiltonian constraint $\tilde{C}$ had no chance to be well-defined in the quantum theory: It is similar to (IIII.1.1.5) due to its density weight +2 . The same factor $1 / \sqrt{\operatorname{det}(q)}$ that was responsible for making (III.1.1.6) finite also makes the original, non-polynomial, unrescaled Hamiltonian constraint $C=\tilde{C} / \sqrt{\operatorname{det}(q)}$ finite. We will now proceed to some details how this is done, avoiding intermediate divergent expressions such as in (III.1.1.9).

The essential steps can already be explained for the first term in (1.2.2.5) so let us drop the "More" term and consider only the integrated first term

$$
C_{E}(N)=\frac{1}{\kappa} \int_{\Sigma} d^{3} x N \frac{F_{a b}^{j} \epsilon_{j k l} E_{j}^{a} E_{l}^{b}}{\sqrt{|\operatorname{det}(E)|}}
$$

Let us introduce a map

$$
R: \Sigma \rightarrow \mathcal{O}(\Sigma) ; x \mapsto R_{x}
$$

where $\mathcal{O}(\Sigma)$ denotes the set of open, compactly supported, connected and simply connected subsets of $\Sigma$ and $R_{x} \in \mathcal{O}(\Sigma)$ is constrained by the requirement that $x \in R_{x}$. We define the volumes of the $R_{x}$ by

$$
V(x):=\operatorname{Vol}\left(R_{x}\right)=\int_{R_{x}} d^{3} y \sqrt{|\operatorname{det}(E)|}(y)
$$

Then, up to a numerical prefactor we may write ([11.1.2.1) in the language of differential forms and in terms of a Poisson bracket as

$$
C_{E}(N)=\frac{1}{\kappa^{2}} \int_{\Sigma} N \operatorname{Tr}(F \wedge\{A, V\})
$$

\section{Exercise III.1.3.}

Verify that (III.1.2.3) is really the volume of $R_{x}$ and ([11.1.2.4).

The reasoning behind (III.1.2.4) was to move the factor $1 / \sqrt{\operatorname{det}(q)}(x)$ from the denominator into the numerator by using a Poisson bracket. This will avoid the $\delta$-distribution in the denominator as in (III.1.1.9) and has the additional advantage that $\sqrt{\operatorname{det}(q)}$ now appears smeared over $R_{x}$ so that one obtains an operator, not a distribution. Thus, the idea is now to replace the function $V(x)$ by the well-defined operator $\widehat{\operatorname{Vol}}\left(R_{x}\right)$ and the Poisson bracket by a commutator divided by $i \hbar$. The only thing that prevents us from doing this is that the operators $A_{a}^{j}, F_{a b}^{j}$ do not exist on $\mathcal{H}_{\text {kin }}$. However, they can be regularized in terms of holonomies as follows:

Given tangent vectors $u, v \in T_{x}(\Sigma)$ we define one parameter homotopies of paths and loops of triangle topology

$$
\epsilon \mapsto p_{\epsilon, x}^{u}, \alpha_{\epsilon, x}^{u v}
$$


respectively with $b\left(p_{\epsilon, x}^{u}\right)=b\left(\alpha_{\epsilon, x}^{u v}\right)=x$ and $\left(\dot{p}_{\epsilon, x}^{u}\right)_{x}=\left(\dot{\alpha}_{\epsilon, x}^{u v}\right)_{x+}=\epsilon u,\left(\dot{\alpha}_{\epsilon, x}^{u v}\right)_{x-}=-\epsilon v$ (left and right derivatives at $x)$. Then for smooth connections $A \in \mathcal{A}$ the Ambrose - Singer theorem tells us that

$$
\lim _{\epsilon \rightarrow 0}\left[A\left(p_{\epsilon, x}^{u}-1\right] / \epsilon=u^{a} A_{a}(x), \quad \lim _{\epsilon \rightarrow 0}\left[A\left(\alpha_{\epsilon, x}^{u v}-1\right] / \epsilon^{2}=u^{a} v^{b} F_{a b}(x) / 2\right.\right.
$$

Exercise III.1.4. Verify (III.1.2.0) by elementary means, using directly the differential equation (II.1.1.1).

Hint:

For sufficiently small $\epsilon$ we have up to $\epsilon^{2}$ corrections $p_{\epsilon, x}^{u}(t)=x+\epsilon t u$ and

$$
\alpha_{\epsilon, x}^{u v}(t)=x+\epsilon\left\{\begin{array}{cc}
t u & t \in[0,1] \\
u / 3+(t-1)(v-u) & t \in[1,2] \\
(3-t) v & t \in[2,3]
\end{array}\right.
$$

Thus, given a triangulation $\tau_{\epsilon}$ of $\sigma$, that is, a decomposition of $\sigma$ into tetrahedra $\Delta$ with base points $v(\Delta)$, edges $p_{I}(\Delta), I=1,2,3$ of $\Delta$ of the type $p_{\epsilon, v}^{u}$ starting at $v$ and triangular loops $\alpha_{I J}(\Delta)=$ $p_{I}(\Delta) \circ a_{I J}(\Delta) p_{J}(\Delta)^{-1}$ of the type $\alpha_{\epsilon, v}^{u v}$ where the $\operatorname{arcs} a_{I J}(\Delta)$ comprise the remaining three edges of $\Delta$, it is easy to show, using ([II.1.2.6), that up to a numerical factor

$$
C_{E}^{\tau_{\epsilon}}(N)=\frac{1}{\kappa^{2}} \sum_{\Delta \in \tau_{\epsilon}} N(v(\Delta)) \sum_{I J K} \epsilon^{I J K} \operatorname{Tr}\left(A \left(\alpha_{I J}(\Delta) A\left(p_{K}(\Delta)\right)\left\{A\left(p_{K}(\Delta)\right)^{-1}, V(v)\right\}\right.\right.
$$

tends to $C_{E}(N)$ as $\epsilon \rightarrow 0$ (in this limit the triangulation gets finer and finer).

\section{Exercise III.1.5.}

Verify this statement.

The expression (โII.1.2.7) can now be readily quantized on $\mathcal{H}_{\text {kin }}$ because holonomies and volume functionals are well-defined operators. However, we must remove the regulator $\epsilon$ in order to arrive at a quantization of (III.1.2.4). Now the regulator can be removed in many inequivalent ways because there is no unique way to refine a triangulation. Moreover, we must specify in which operator topology $\hat{C}^{\tau_{\epsilon}}(N)$ converges. The discussion of these issues is very complicated and the interested reader is referred to [48] for the detailed arguments that lead to the following solution:

i) Triangulation

First of all we define the operator explicitly on the SNW basis $T_{s}$. In order for the refinement limit to be non-trivial, it turns out that the triangulation must be refined in such a way that $\gamma(s) \subset \tau_{\epsilon}$ for sufficiently small $\epsilon$. This happens essentially due to the volume operator which has non-trivial action only at vertices of graphs. Thus the refinement must be chosen depending on $s$. This is justified because classically all refinements lead to the same limit. One might worry that this does not lead to a linear operator, however, this is not the case because it is defined on a basis.

\section{ii) Operator Topology}

The limit $\epsilon \rightarrow 0$ exists in the following sense:

Let $\mathcal{D}_{\text {Diff }}^{*} \subset \mathcal{D}_{\text {kin }}^{*}$ be the space of solutions of the diffeomorphism constraint. We say that a family of operators $\hat{O}_{\epsilon}$ converges to an operator $\hat{O}$ on $\mathcal{H}_{\text {kin }}$ in the uniform - weak - Diff-topology provided that for each $\delta>0$ and for each $l \in \mathcal{D}_{\text {Diff }}^{*}, f \in \mathcal{D}_{\text {kin }}$ there exists $\epsilon(\delta)>0$ independent of $l, f$ such that

$$
\left|l\left(\left[\hat{O}_{\epsilon}-\hat{O}\right] f\right)\right|<\delta \quad \forall \epsilon<\epsilon(\delta)
$$




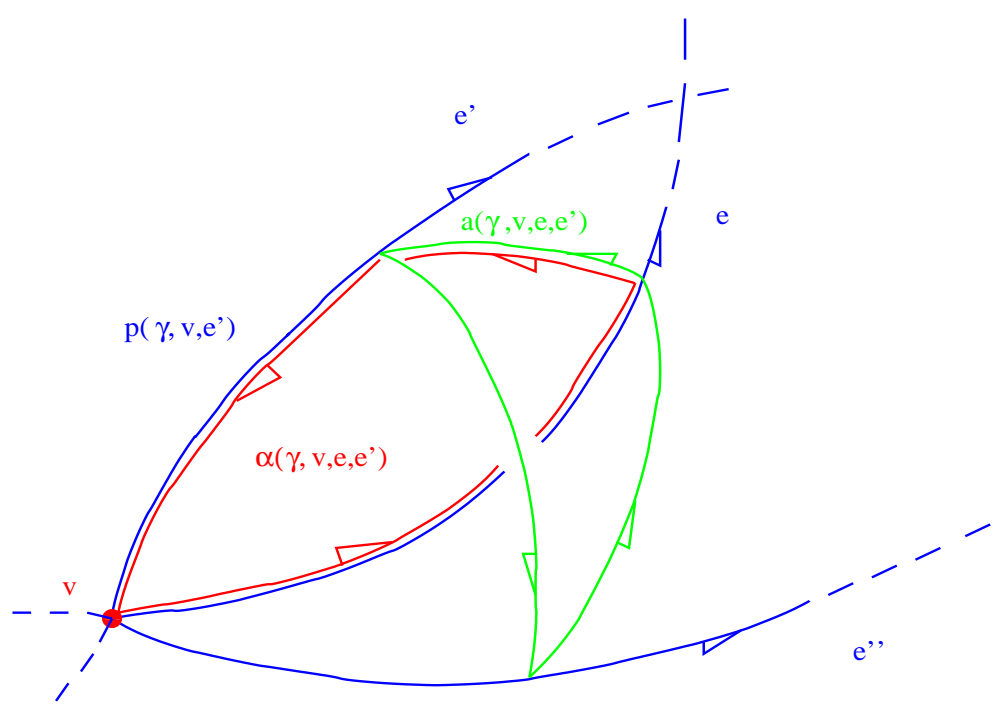

Figure 11: Meaning of the loop, path and arc assignment of the Hamiltonian constraint. Notice how a tetrahedron emerges from those objects, making the link with the triangulation. The broken lines indicate possible other edges or continuations thereof.

This topology is of course motivated by physical considerations: Since the operator is unbounded, the uniform (i.e. operator norm) topology is too strong. The strong or weak topologies (pointwise convergence in Hilbert space norm or as matrix elements) give a trivial (zero) limit (excercise !). Thus one is naturally led to ${ }^{*}$ topologies. The maximal dual space on which to build a topology would be $\mathcal{D}_{k i n}^{*}$ but one can check that the limit does not exist even pointwise in $\mathcal{D}_{\text {kin }}^{*}$. Thus one is looking for suitable subspaces thereof. The natural, physically motivated choice is, of course, the space $\mathcal{D}_{\text {Diff }}^{*}$ which is singled out by the spatial diffeomorphism constraint. The reason for why have required uniform convergence in (III.1.2.8) is that this excludes the existence of the limit for larger spaces $\mathcal{D}_{\text {Diff }}^{*} \subset \mathcal{D}_{\star}^{*} \subset \mathcal{D}_{\text {kin }}^{*}$.

The end result is

$$
\begin{gathered}
\hat{C}_{E}^{\dagger}(N)=m_{P} \sum_{v \in V(\gamma(s))} N(v) \quad \sum_{e, e^{\prime}, e^{\prime \prime} \in E(\gamma(s))} \times \\
e \cap e^{\prime} \cap e^{\prime \prime}=\{v\} \\
\times \quad\left\{\operatorname{Tr}\left(\left[A\left(\alpha_{\gamma(s), v, e, e^{\prime}}\right)-\left(A\left(\alpha_{\gamma(s), v, e, e^{\prime}}\right)\right)^{-1}\right] A\left(p_{\gamma(s), v, e^{\prime \prime}}\right)\left[A\left(p_{\gamma(s), v, e^{\prime \prime}}\right)^{-1}, \hat{V}_{v}\right]\right)\right. \\
\left.\quad+\text { cyclic permutation in }\left\{e, e^{\prime}, e^{\prime \prime}\right\}\right\} T_{s}
\end{gathered}
$$

The meaning of the loops $\alpha_{\gamma, v, e, e^{\prime}}$ and paths $p_{\gamma, v, e^{\prime \prime}}$ that appears in this sum over vertices and triples of edges incident at them is maybe best explained in the following figure 11. Their precise specification makes use of the axiom of choice and is diffeomorphism covariant, that is, for $\varphi \in \operatorname{Diff}^{\omega}(\Sigma)$, e.g. the loops $\alpha_{\gamma, v, e, e^{\prime}}$ and $\alpha_{\varphi(\gamma), \varphi(v), \varphi(e), \varphi\left(e^{\prime}\right)}$ are analytically diffeomorphic. Moreover, the $\operatorname{arcs} a_{\gamma, v, e, e^{\prime}}$ defined by

$$
\alpha_{\gamma, v, e, e^{\prime}}=p_{\gamma, v, e} \circ a_{\gamma, v, e, e^{\prime}} \circ p_{\gamma, v, e^{\prime}}^{-1}
$$

are such that also $\gamma \cup a_{\gamma, v, e, e^{\prime}}$ and $\varphi(\gamma) \cup a_{\varphi(\gamma), \varphi(v), \varphi(e), \varphi\left(e^{\prime}\right)}$ are anlytically diffeomorphic. The adjoint in (III.1.2.9) is due to the fact that $C_{E}(N)$ is classically real-valued, so we are quantizing $\overline{C_{E}(N)}$ as well. The operator (III.1.2.9) is not symmetric, however, its adjoint is densely defined on $\mathcal{D}_{k i n}$ and it is therefore closable. Usually one requires real valued functions to become self-adjoint operators 
because then by the spectral theorem the spectrum (possible measurement values) is a subset of the real line. However, this argument is void when we are only interested in the kernel of the operator ("zero eigenvalue").

\section{Exercise III.1.6.}

Verify that $\hat{C}_{E}^{\dagger}(N)$ is not symmetric but it is, together with $\hat{C}_{E}(N)$, densely defined on $\mathcal{D}_{\text {kin }}$. Show that if real valued constraints $C_{I}$ form a Poisson algebra $\left\{C_{I}, C_{J}\right\}=f_{I J}{ }^{K} C_{K}$ with non-trivial, real valued structure functions such that $\left\{f_{I J}{ }^{K}, C_{K}\right\}_{\left\{C_{L}=0\right\}} \neq 0$, then $\hat{C}_{I}, \hat{f}_{I J}{ }^{K}$ must not be both symmetric in order for the quantum algebra to be free of anomalies. Conclude that the failure of (111.1.2.9 to be symmetric is likely to be required for reasons of consistency.

The fact the loop $\alpha_{\gamma, v, e, e^{\prime}}$ is not shrunk to $v$ as one would expect is of course due to our definition of convergence, in fact, an arbitrary loop assignment $\left(\gamma, v, e, e^{\prime}\right) \mapsto \alpha_{\gamma, v, e, e^{\prime}}$ that has the same diffeomorphism invariant characteristics is allowed, again because in a diffeomorphism invariant theory there is no notion of "closeness" of $\alpha_{\gamma, v, e, e^{\prime}}$ to $v$. Notice that the operator $\hat{C}_{E}(N)$ is defined on $\mathcal{H}_{k i n}$ using the axiom of choice and not on diffeomorphism invariant states as it is sometimes misleadingly stated in the literature [50]. In fact, it cannot be because the dual operator $\hat{C}_{E}^{\prime}(N)$ defined by

$$
\left[\hat{C}_{E}^{\prime}(N) l\right](f):=l\left(\hat{C}_{E}^{\dagger}(N) f\right)
$$

for all $f \in \mathcal{D}_{\text {kin }}, l \in \mathcal{D}_{\text {kin }}^{*}$ does not preserve $\mathcal{D}_{\text {Diff }}^{*}$ as is expected from the the classical Poisson algebra $\{V, C\} \propto C \neq V$. If one wants to take this dual point of view then one is forced to introduce a larger space $\mathcal{D}_{\star}^{*}$ which is preserved but which does not solve the diffeomorphism constraint and is therefore unphysical. This has unnecessarily given rise to a large amount of confusion in the literature and should be abandomed.

As we have said, the loop assignment is to a very large extent arbitrary at the level of $\mathcal{H}_{\text {kin }}$ and represents a serious quantization ambiguity, it cannot even be specified precisely because we are using the axiom of choice. However, at the level of $\mathcal{H}_{\text {phys }}$ this ambiguity evaporates to a large extent because all choices that are related by a diffeomorphism result in the same solution space to all constraints defined by elements $l \in \mathcal{D}_{\text {Diff }}^{*}$ which satisfy in addition

$$
\left[\hat{C}_{E}^{\prime}(N) l\right](f)=l\left(\hat{C}_{E}^{\dagger}(N) f\right)=0 \forall N \in C_{0}^{\infty}(\Sigma), f \in \mathcal{D}_{k i n}
$$

where $C_{0}^{\infty}(\Sigma)$ denotes the smooth functions of compact support. Thus the solution space $\mathcal{D}_{\text {phys }}^{*}$ will depend only on the spatially diffeomorphism invariant characteristics of the loop assignment which can be specified precisely [48], it essentially characterizes the amount by which the arcs knot the original edges of the graph. Besides this remaining ambiguity there are also factor ordering ambiguities but no singularities some of which are discussed in [51].

Let us list without proof some of the properties of this operator:

i) Matter Coupling

Similar Techniques can be applied to the case of (possibly supersymmetric) matter coupled to GR 48.

ii) Anomaly - Freeness

The constraint algebra of the Hamiltonian constraint with the spatial diffeomorphism constraint and among each other is mathematically consistent. From the classical constraint algebra $\{V, C\} \propto C$ we expect that $\hat{V}(\varphi) \hat{C}_{E}^{\dagger}(N) \hat{V}(\varphi)^{-1}=\hat{C}_{E}\left(\varphi^{*} N\right)$ for all diffeomorphisms $\varphi$. However, this is just the statement of the loop assignment being diffeomorphism covariant 
which can be achieved by making use of the axiom of choice. Next, from $\{C, C\} \propto V$ we expect that the dual of $\left[\hat{C}_{E}^{\dagger}(N), \hat{C}_{E}^{\dagger}\left(N^{\prime}\right)\right]=\left[\hat{C}_{E}\left(N^{\prime}\right), \hat{C}_{E}(N)\right]^{\dagger}$ annihilates the elements of $\mathcal{D}_{\text {Diff }}^{*}$. This can be explicitly verified [48]. We stress that $\left[\hat{C}_{E}^{\dagger}(N), \hat{C}_{E}^{\dagger}\left(N^{\prime}\right)\right]$ is not zero, the algebra of Hamiltonian constraints is not Abelean as it is sometimes misleadingly stated in the literature. The commutator is in fact explicitly proportional to a diffeomorphism.

iii) Physical States

There is a rich space of rigorous solutions to (III.1.2.12) and a precise algorithm for their construction has been developed [48].

iv) Intuitive Picture

The Hamiltonian constraint acts by annihilating and creating spin degrees of freedom and therefore the dynamical theory obtained could be called "Quantum Spin Dynamics (QSD)" in analogy to "Quantum Chromodynamics (QCD)" in which the Hamiltonian acts by creating and annihilating colour degrees of freedom. In fact we could draw a crude analogy to Fock space terminology as follows: The (perturbative) excitations of QCD carry a continuous label, the mode number $k \in \mathbb{R}^{3}$ and a discrete label, the occupation number $n \in \mathbb{N}$ (and others). In QSD the continuous labels are the edges $e$ and the discrete ones are spins $j$ (and others). So we have something like a non - linear Fock representation in front of us.

Next, when solving the Hamiltonian constraint, that is, when integrating the Quantum Einstein Equations, one realizes that one is not dealing with a (functional) partial differential equation but rather with a (functional) partial difference equation. Therefore, when understanding coordinate time as measured how for instance volumes change, we conclude that also time evolution is necessarily discrete. Such discrete time evolution steps driven by the Hamiltonian constraint assemble themselves into what nowadays is known as a spin foam. A spin foam is a four dimensional complex of two dimensional surfaces where each surface is to be thought of as the world sheet of an edge of a SNW and it carries the spin that the edge was carrying before it was evolved[?

Another way of saying this is that a spin foam is a complex of two-surfaces labelled by spins and when cutting a spin foam with a spatial three-surface $\Sigma$ one obtains a SNW. If one uses two such surfaces $\Sigma_{t}, \Sigma_{t+T_{P}}$ where $T_{P}=\ell_{P} / c$ is the Planck time then one rediscovers the discrete time evolution of the Hamiltonian constraint. These words are summarized in figure 12.

While these facts constitute a promising hint that the Hilbert space $\mathcal{H}_{\text {kin }}$ could in fact support the quantum dynamics of $G R$, there are well-taken concerns about the physical correctness of the operator $\hat{C}_{E}^{\dagger}(N)$ :

The problem is that one would like to see more than that the commutator of two dual Hamiltonian constraints annihilates diffeomorphism invariant states, one would like to see something of the kind

$$
\left[\hat{C}_{E}^{\dagger}(N), \hat{C}_{E}^{\dagger}\left(N^{\prime}\right)\right]=i \ell_{P}^{2}\left[\int_{\Sigma} d^{3} x\left[N N_{, a}^{\prime}-N_{, a} N^{\prime}\right] q^{a} b V_{b}\right]
$$

The reason for this is that then one would be sure that $\hat{C}_{E}^{\dagger}(N)$ generates the correct quantum evolution. While this requirement is not necessary, it is certainly sufficient and would be reassuring].

\footnotetext{
${ }^{7}$ Thus, a spin foam model can be thought of as a background independent string theory!

${ }^{8}$ Example: Suppose that $C_{a}$ are the angular momentum components for a particle in in $\mathbb{R}^{3}$ with classical constraint
} 


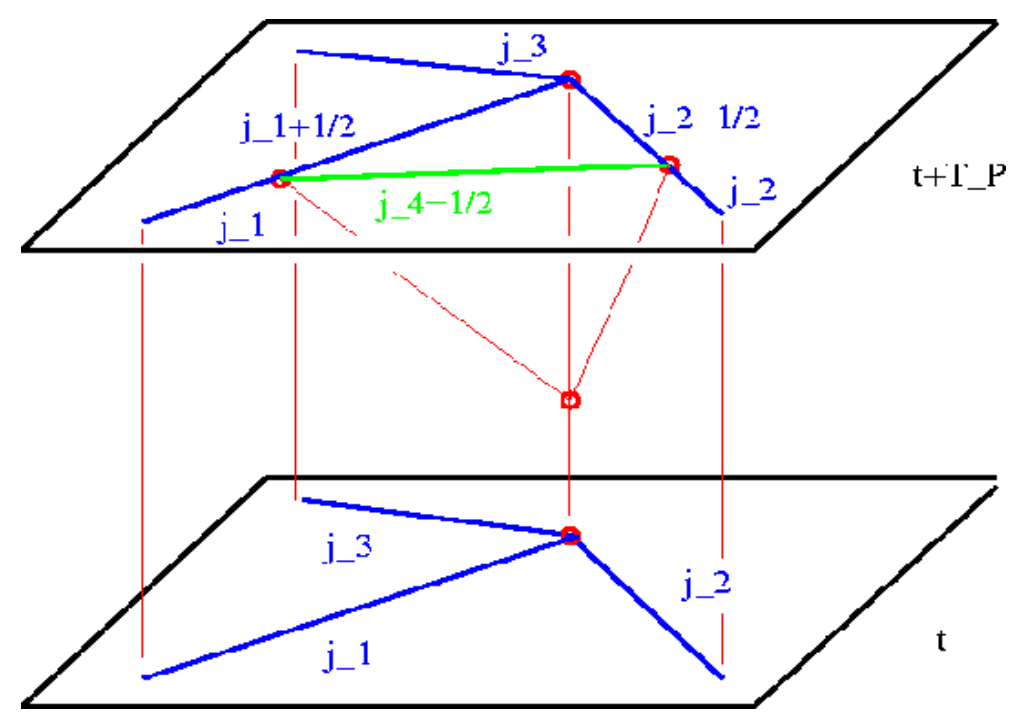

Figure 12: Emergence of a spin foam from a SNW by the action of the Hamiltonian constraint

There are two obstacles that prevent us from rewriting the left hand side of ([II.1.2.13) in terms of the right hand side.

1)

The one parameter groups $s \mapsto \hat{V}\left(\varphi_{s}^{u}\right)$ of unitarities where $\varphi_{s}^{u}$ are the one parameter groups of diffeomorphisms defined by the integral curves of a vector field $u$ are not weakly continuous, therefore a self-adjoint generator $\hat{V}(u)$ that we would like to see on the right hand side of (III.1.2.13) simply does not exist.

\section{Exercise III.1.7.}

Recall Stone's theorem about the existence of the self-adjoint generators of weakly continuous oneparameter unitary groups and verify that $\hat{V}\left(\varphi_{s}^{u}\right)$ is not weakly continuous on $\mathcal{H}_{\text {kin }}$.

2)

One can quantize the right hand side of (111.1.2.13) by independent means and it does annilate $\mathcal{D}_{\text {Diff }}^{*}$ [48], however, that operator does not resemble the left hand side in any obvious way. The reason for this is that even classically it takes a DinA4 page of calculation in order to rewrite the Poisson bracket $\left\{C_{E}(N), C_{E}\left(N^{\prime}\right)\right\}$ as in (III.1.2.13) with $V_{a}$ given by (I.2.2.5). The manipulations that must be performed in order to massage the Poisson bracket into the desired form involve a) integrations by part, b) writing $F_{a b}$ in terms of $A_{a}$, c) derivatives of $\sqrt{\operatorname{det}(q)}$, d) multiplying fractions by functions in both numerator and denominator, e) symmetry arguments in order to see that certain tems cancel etc. (exercise !). These steps are obviously difficult to perform with operators.

In summary, there is no mathematical inconsistency, however, there are doubts about the physical correctness of the Hamiltonian constraint operator presently proposed although no proof exists so far that it is necessarily wrong. In order to make progress on this issue, it seems that we need to develop first a semiclassical calculus for the theory, more precisely, we need coherent states so that expectation values of operators and their commutators can be replaced, up to $\hbar$ corrections, by their classical values and Poisson brackets respectively for which then the manipulations listed in 2)

algebra $\left\{C_{a}, C_{b}\right\}=\epsilon_{a b c} C_{c}$. Introduce polar coordinates and define the non-self adjoint operators $\hat{C}_{1}=i \hbar \partial / \partial \theta, \hat{C}_{2}=$ $i \hbar \partial / \partial \phi, \hat{C}_{3}=0$. Then the quantum constraint algebra is Abelean and does not at all resemble the classical one, however, the physical states are certainly the correct ones, functions that depend only on the radial coordinate. 
above can be carried out. If that is possible, and the outcome of these calculations is the expected one, possibly after changing the operator by making use of the available quantization ambiguities, then one would be able to claim that one has indeed constructed a quantum theory of GR with the correct classical limit. Only then can one proceed to solve the theory, that is, to construct solutions, the physical inner product and the Dirac observables. The development of a semiclassical calculus is therefore one of the "hot" research topics at the moment.

Another way to get confidence in the quantization method applied to the Hamiltonian constraint is to study model systems for which the answer is known. This has been done for $2+1$ gravity [48] and for quantum cosmology to which we turn in the next section.

\section{III.2 Loop Quantum Cosmology}

\section{III.2.1 A New Approach To Quantum Cosmology}

The traditional approach to quantum cosmology consists in a so-called mini - superspace quantization, that is, one imposes certain spacetime Killing symmetries on the metric, plugs the symmetric metric into the Einstein Hilbert action and obtains an effective action which depends only on a finite number of degrees of freedom. Then one canonically quantizes this action. Thus one symmetrizes before quantization. These models are of constant interest and have natural connections to inflation. See e.g. [52 for recent reviews.

What is not perfect about these models is that 1) not only do they switch off all but an infinite number of degrees of freedom, but 2) also the quantization method applied to the reduced model usually is quite independent from that applied to the full theory. A fundamental approach to quantum cosmology will be within the full theory and presumably involves the construction of semiclassical physical states whose probability amplitude is concentrated on, say a Friedmann - Robertson Walker (FRW) universe. This would cure both drawbacks 1) and 2). At the moment we cannot really carry out such a programme since the construction of the full theory is not yet complete. However, one can take a more modest, hybrid approach, where while dealing only with a finite number of degrees of freedom one takes over all the quantization machinery from the full theory! Roughly speaking, one works on the space $\mathcal{H}_{\text {kin }}$ of the full theory but considers only states therein which satisfy the Killing symmetry. Hence one symmetrizes after quantization which amounts to considering only a finite subset of holonomies and fluxes. This has the advantage of leading to a solvable model while preserving pivotal structures of the full theory, e.g. the volume operator applied to symmetric states will still have a discrete spectrum as in the full theory while in the traditional approaches it is continuous. Such a programme has been carried out in great detail by Bojowald in a remarkable series of papers [53] and his findings are indeed spectacular, should they extend to the full theory: It turns out that the details of the quantum theory are drastically different from the traditional minisuperspace approach. In what follows we will briefly describe some of these results, skipping many of the technical details.

\section{III.2.2 Spectacular Results}

Consider the FRW line element (in suitable coordinates)

$$
d s^{2}=-d t^{2}+R(t)^{2}\left[\frac{d r^{2}}{1-k r^{2}}+r^{2} d \Omega_{2}^{2}\right]=:-d t^{2}+R(t)^{2} q_{a b}^{0} d x^{a} d x^{b}
$$




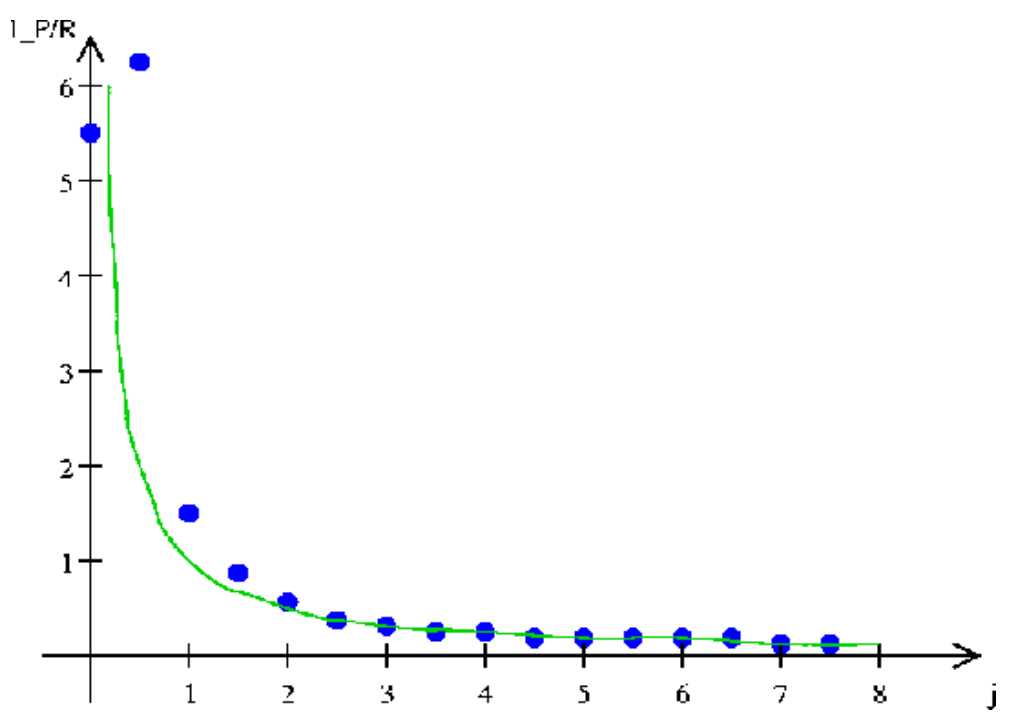

Figure 13: Spectrum of the inverse scale factor

The universe is closed/flat/open for $k=1 / 0 /-1$. The only dynamical degree of freedom left is the so-called scale factor $R(t)$ which describes the size of the universe and its conjugate momentum. The classical big bang singularity corresponds to the fact that the Einstein equations predict that $\lim _{t \rightarrow 0} R(t)=0$ at which the metric (III.2.2.1) becomes singular and the inverse scale factor $1 / R(t)$ blows up (the curvature will be $\propto 1 / R(t)^{2}$ so this singularity is a true curvature singularity).

We are interested in whether the curvature singularity $1 / R \rightarrow \infty$ exists also in the quantum theory. To study this we notice that for (III.1.1.3) $\operatorname{det}(q)=R^{6} \operatorname{det}\left(q^{0}\right)$. Hence, up to a numerical factor this question is equivalent to the question whether the operator corresponding to $1 / \sqrt[6]{\operatorname{det}(q)}$, when applied to symmetric states, is singular or not. However, we saw in the previous section that one can trade a negative power of $\operatorname{det}(q)$ by a Poisson bracket with the volume operator. In [53] precisely this, for the Hamiltonian constraint, essential quantization technique is applied which is why this model tests some aspects of the quantization of the Hamiltonian constraint. Now it turns out that this operator, applied to symmetric states, leads to an operator $\frac{\widehat{1}}{R}$ which is diagonalized by (symmetric) SNWF's and the spectrum is bounded! In figure 13 we plot the qualitative behaviour of the eigenvalues $\ell_{P} \lambda_{j}$ as a function of $j$ where $j$ is the spin label of a gauge invariant SNWF with a graph consisting of one loop only (that only such states are left follows from a systematic analysis which defines what a symmetric SNWF is). One can also quantize the operator $\hat{R}$ and one sees that its eigenvalues are essentially given by $j \ell_{P}$ up to a numerical factor. Thus the classical singularity corresponds to $j=0$ and one expects the points $\lambda_{j} \ell_{p}$ at the values $1 / j$ on the curve $\ell_{P} / R$. Evidently the spectrum is discrete (pure point) and bounded, at the classical singularity it is finite. In other words, the quantum universe never decreases to zero size. For larger $j$, in fact already for $R$ of the order of ten Planck lengths and above, the spectrum follows the classical curve rather closely hinting at a well-behaved classical limit (correspondence principle).

Even more is true: One can in fact quantize the Hamiltonian constraint by the methods of the previous section and solve it exactly. One obtains an eighth order difference equation (in $j$ ). The solution therefore depends non-trivially on the initial condition. What is surprising, however, is the fact that only one set of initial conditions leads to the correct classical limit, thus in loop quantum cosmology initial conditions are derived rather than guessed. One can even propagate the quantum Einstein equations through the classical singularity and arrives at the picture of a bouncing universe.

Finally one may wonder whether these results are qualitatively affected by the operator ordering 
ambiguities of the Hamiltonian constraint. First of all one finds that these results hold only if one orders the loop in (III.1.2.9) to the left of the volume operator as written there. However, one is not forced to work with the holonomy around that loop in the fundamental representation of $S U(2)$, there is some flexibility [51] and one can choose a different one, say $j_{0}$. It turns out that the value $j_{0}$ influences the onset of classical behaviour, that is, the higher $j_{0}$ the higher the value $j\left(j_{0}\right)$ from which on the spectrum in figure 13 lies on the curve $1 / j$. Now this is important when one copuples, say scalar matter because the operator $\frac{\widehat{1}}{R}$ enters the matter part of the Hamiltonian constraint and modifies the resulting effective equation for $R(t)$ in the very early phase of the universe and leads to a quantum gravity driven inflationary period whose duration gets larger with larger $j_{0}$ !

Thus, loop quantum cosmology not only confirms aspects of the quantization of the Hamiltonian constraint but also predicts astonishing deviations from standard quantum cosmology which one should rederive in the full theory.

\section{III.3 Path Integral Formulation: Spin Foam Models}

\section{III.3.1 Spin Foams from the Canonical Theory}

Spin Foam models are the fusion of ideas from topological quantum field theories and loop quantum gravity, see e.g. [54 for a review, especially the latest, most updated one by Perez. The idea that connects these theories is actually quite simple to explain at an heuristic level:

If we forget about 1) all functional analytic details, 2) the fact that the operator valued distributions corresponding to the Hamiltonian constraint $\hat{C}(x)$ do not mutually commute for different $x \in \sigma$ and 3) that the Hamiltonian constraint operators $\hat{C}(N)$ are certainly not self-adjoint, at least as presently formulated, then we can formally write down the complete space of solutions to the Hamiltonian constraint as a so-called "rigging map" (see e.g. [1])

$$
\bar{\eta}: \mathcal{D}_{\text {kin }} \rightarrow \mathcal{D}_{\text {phys }}^{*} ; f \mapsto \delta[\hat{C}] f:=\left[\prod_{x \in \Sigma} \delta(\hat{C}(x)) f\right]
$$

(where $\bar{\eta}=$ c.c $\cdot \eta$ is the complex conjugate of the actual anti-linear rigging map). Here the $\delta$-distribution of an operator is defined via the spectral theorem (assuming the operator to be self-adjoint). Notice that we do not need to order the points $x \in \sigma$ as we assumed the $\hat{C}(x)$ to be mutually commuting for the moment and only under this assumption it is true that, at least formally $\bar{\eta}[f]\left(\hat{C}(N) f^{\prime}\right)=0$ (exercise) $]^{\mathbb{f}}$. Now we use the formula $\delta(x)=\int_{\mathbb{R}} \frac{d k}{2 \pi} e^{i k x}$ to write the functional $\delta$-distribution $\delta[\hat{C}]$ as a path integral

$$
\delta[\hat{C}]=\int_{\mathcal{N}^{\prime}}[D N] e^{i \hat{C}(N)}
$$

where we have neglected an infinite constant as usual in this formal business. Here $\mathcal{N}^{\prime}$ is the space of lapse functions at a fixed time. Let us introduce also the space of lapses with arbitray time dependence $\mathcal{N}_{t_{1}, t_{2}}$ in $t \in\left[t_{1}, t_{2}\right]$. Then, up to an infinite constant one can verify that

$$
\delta[\hat{C}]=\int_{\mathcal{N}_{t_{1}}^{t_{2}}}[D N] e^{i \int_{t_{1}}^{t_{2}} d t \int_{\Sigma} d^{3} x N(x, t) \hat{C}(x)}
$$

\footnotetext{
${ }^{9}$ At an even more formal level $\bar{\eta}[f]$ is also a solution in the non-commuting case if, as is the case with the currently proposed $\hat{C}$, the algebra with the spatial diffeomorphism constraint closes
} 
The rigging map machinery then tells us that the scalar product on the image of the rigging map is simply given by

$$
<\bar{\eta}(f), \bar{\eta}\left(f^{\prime}\right)>_{p h y s}:=<f, \bar{\eta}\left(f^{\prime}\right)>_{k i n}=\int_{\mathcal{N}_{t_{1}}^{t_{2}}}[D N]<f, e^{i \int_{t_{1}}^{t_{2}} d t \hat{C}\left(N_{t}\right)} f^{\prime}>_{k i n}
$$

This formula looks like a propagator formula, that is, like a transition amplitude between an initial state $f^{\prime}$ on $\Sigma_{t_{1}}$ and a final state $f$ on $\Sigma_{t_{2}}$ after a multi-fingered time evolution generated by $\hat{C}\left(N_{t}\right)$. In fact, if we use the Taylor expansion of the exponential function and somehow regularize the path integral then the expansion coefficients $<T_{s}, \hat{C}\left(N_{t}\right)^{n} T^{\prime} s^{\prime}>_{k i n}$ can be interpreted as probability amplitude of the evolution of the SNW state $T_{s^{\prime}}^{\prime}$ to reach the SNW state $T_{s}$ after $n$ time steps (recall figure 12).

Now by the usual formal manipulations that allow us to express a unitary operator $e^{i\left(t_{2}-t_{1}\right) \hat{H}}$ as a path integral over the classical pase space $\mathcal{M}$ (the rigorous version of which is the Feynman $-\mathrm{Kac}$ formula, e.g. [56]) one can rewrite (III.3.1.4) as

$$
<\bar{\eta}(f), \bar{\eta}\left(f^{\prime}\right)>_{p h y s}=\int[D N D \vec{N} D \Lambda D A D E]<f, e^{i S} f^{\prime}>_{k i n}
$$

where $S$ is the Einstein-Hilbert action written in canonical form in terms of the variables $A, E$, that is

$$
S=\int_{\mathbb{R}} d t \int_{\Sigma} d^{3} x\left\{\dot{A}_{a}^{j} E_{j}^{a}-\left[-\Lambda^{j} G_{j}+N^{a} V_{a}+N C\right]\right\}
$$

and we have simultaneously included also projections on the space of solutions to the Gauss and vector constraint. Now the action ([II.3.1.6) is the $3+1$ split of the following covariant action

$$
S=\int_{M}\left\{\Omega_{I J} \wedge\left[\epsilon^{I J K L}-\beta^{-1} \eta^{I K} \eta^{J K}\right] e_{K} \wedge e_{L}\right\}
$$

discovered in [57] where $\beta$ is the Immirzi parameter. Here $\Omega_{I J}$ is the (antisymmetric) curvature two-form of an (antisymmetric) $S L(2, \mathbb{C})$ connection one-form $\omega_{I J}$ with Lorentz indices $I, J, K, . .=$ $0,1,2,3, \eta$ is the Minkowski metric and $e^{I}$ is the co-tetrad one-form. The first term in (III.3.1.7) is called the Palatini action while the second term is topological (a total differential modulo the equations of motion). The relationn between the four-dimensional fields $\omega_{\mu}^{I J}, e_{\mu}^{I}$ (40 components) and the three-dimensional fields $A_{a}^{j}, E_{j}^{a}, \Lambda^{j}, N, N^{a}$ (25 components) can only be established if certain so-called second class constraints [20] are solved.

\section{III.3.2 Spin Foams and BF - Theory}

Thus, it is formally possible to write the inner product between physical states as a covariant path integral for the classical canonical action and using only the kinematical inner product, thus providing a bridge between the covariant and canonical formalism. However, this bridge is far from being rigorously established as we had to perform many formal, unjustified manipulations. Now rather than justifying the steps that lead from $\hat{C}$ to (III.3.1.5) one can turn the logic upside down and start from a manifestly covariant formulation and derive the canonical formultion. This is the attitude that one takes among people working actively on spin foam models. Thus, let us forget about the topological term in ([II.3.1.7) and consider only the Palatini term. Then the Palatini action has precisely the form of a $\mathrm{BF}$ - action

$$
S_{B F}=\int_{M} \Omega_{I J} \wedge B^{I J}
$$


just that the (antisymmetric) two-form field $B^{I J}$ is not arbitrary (it would have 36 independent components), it has to come from a tetrad with only 16 independent components, that is, it has to be of the form $\epsilon^{I J K L} e_{K} \wedge e_{L}$.

\section{Exercise III.3.1.}

Show that the condition that $B$ comes from a tetrad is almos $\mathbb{0}$ equivalent to the simplicity constraint

$$
\epsilon_{I J K L} B_{\mu \nu}^{I J} B_{\rho \sigma}^{K L}=c \epsilon_{\mu \nu \rho \sigma}
$$

for some spacetime scalar density $c$ of weight one.

The reasoning is now as follows: BF - theory without the constraint (III.3.2.2) is a topological field theory, that is, it has no local degrees of freedom. Therefore quantum BF - theory is not really a QFT but actually a quantum mechanical system and can therefore be handled much more easily than gravity. Let us now write an action equivalent to the Palatini action given by

$$
\begin{aligned}
S_{P}^{\prime}[\omega, B, \Phi] & =S_{B F}[\omega, B]+S_{I}[B, \Phi] \\
S_{I}[B, \Phi] & :=\int_{M} \Phi^{\mu \nu \rho \sigma} \epsilon_{I J K L} B_{\alpha \beta}^{I J} B_{\gamma \delta}^{K L}\left[\delta_{\mu}^{\alpha} \delta_{\nu}^{\beta} \delta_{\rho}^{\gamma} \delta_{\sigma}^{\delta}-\frac{1}{4 !} \epsilon^{\alpha \beta \gamma \delta} \epsilon_{\mu \nu \rho \sigma}\right]
\end{aligned}
$$

where the Lagrange multiplier $\Phi^{\mu \nu \rho \sigma}$ [58] is a four dimensional tensor density of weight one, symmetric in the index pairs $(\mu \nu)$ and $(\rho \sigma)$ and antisymmetric in each index pair. Thus, $\Phi$ has $(6 \cdot 7) / 2=21$ independent components of which the totally skew component is projected out in ([II.3.2.3), leaving us with $36-16=20$ independent components. Hence the Euler Lagrange equations for $\Phi$ precisely delete the amount of unwanted degrees of freedom in $B$ and impose the simplicity constraint. Hence, classically $S_{P}^{\prime}[\Omega, B, \Phi]$ and $S_{P}[\Omega, e]$ are equivalent. Thus, if we write a path integral for $S_{P}^{\prime}$ and treat the Lagrange multiplier term $S_{I}$ in (III.3.2.3) as an interaction Lagrangean (a perturbation) to BF - theory, then we can make use of the powerful techniques that have been developed for the path integral quantization for BF - theory and its perturbation theory.

\section{Exercise III.3.2.}

i) Write the Euler Lagrange equations for BF - theory and conclude that the solutions consist of flat connections $\omega$ and gauge invariant $B$ - fields. Conclude that $\omega$ can be gauged to zero by $S L(2, \mathbb{C})$ transformations locally and that then $B$ is closed, that is, locally exact by Poincaré's theorem. Now, verify that the BF-action is not only invariant under local $S L(2, \mathbb{C})$-transformations but also under

$$
B^{I J}=\mapsto B^{I J}+(D \wedge \theta)^{I J}=B^{I J}+d \theta^{I J}+\omega^{I}{ }_{K} \wedge \theta^{K J}+\theta^{I K} \wedge \omega_{K}{ }^{J}
$$

for some $\operatorname{sl}(2, \mathbb{C})$ valued one - form $\theta$ and that therefore also $B$ can be gauged to zero locally.

Hint:

Use the Bianchi identity for $\Omega$.

ii) Perform the Legendre transformation and conclude that there are as many first class constraints as canonical pairs so that again at most a countable number of global degrees of freedom can exist.

One may wonder how it is possible that a theory with less kinematical degrees of freedom has more dynamical (true) degrees of freedom. The answer is that BF - theory has by far more symmetries than the Palatini theory, thus when constraining the number of degrees of freedom we are freezing more symmetries than we deleted degrees of freedom.

\footnotetext{
${ }^{10}$ Another solution is $B^{I J}=e^{I} \wedge e^{J}$ but this possibility is currently not discussed.
} 
Let us now discuss how one formulates the path integral corresponding to the action (III.3.2.3). It is formally given by

$$
K_{P}\left(\Sigma_{t_{1}}, \Sigma_{t_{2}}\right)=\int[D \omega D B D \Phi] e^{i S_{P}^{\prime}[\omega, B, \Phi]}
$$

where $\Sigma_{t 1}, \Sigma_{t_{2}}$ denote suitable boundary conditions specified in more detail below. Suppose we set $\Phi=0$, then (III.3.2.5) is a path integral for BF - theory and the integral over $B$ results in the functional $\delta$-distribution $\delta[\Omega]$ imposing the flatness of $\omega$. Now flatness of a connection is equivalent to trivial holonomy along contractable loops by the Ambrose - Singer theorem. If one regularizes the path integral by introducing a triangulation $\tau$ of $M$, then $\delta[F]$ can be written as $\prod_{\alpha} \delta(\omega(\alpha), 1)$ where the product is over a generating system of independent, contractible loops in $\tau$ and $\delta(\omega(\alpha), 1)$ denotes the $\delta$-distribution on $S L(2, \mathbb{C})$ with respect to the Haar measure. Since $S L(2, \mathbb{C})$ is a non-compact group, the $\delta$-distribution is a direct integral over irreducible, unitary representations rather than a direct sum as it would be the case for compact groups (Peter\&Weyl theorem). Such representations are infinite dimensional and are labelled by a continuous parameter $\rho \in \mathbb{R}_{0}^{+}$and a discrete parameter $n \in \mathbb{N}_{0}^{+}$. Thus, one arrives at a triangulated a spin foam model: For a fixed triangulation one integrates (sums) over all possible "spins" $\rho(n)$ that label the generating set of loops (equivalently: the faces that they enclose) of that triangulated four manifold. The analogy with the state sum models for TQFT's is obvious.

Now what one does is a certain jump, whose physical implication is still not understood: Instead of performing perturbation theory in $S_{I}$ one argues that formally integrating over $\Phi$ and thus imposing the simplicity constraint is equivalent to the restriction of the direct integral that enters the $\delta$-distributions to simple representations, that is, representations for which either $n=0$ or $\rho=0$. In other words, one says that the triangulated Palatini path integral is the same as the triangulated $\mathrm{BF}$ path integral restricted to simple representations. To motivate this argument, one notices that upon canonical quantization of BF theory on a triangulated manifold the $B$ field is the momentum conjugate to $\omega$ and if one quantizes on a Hilbert space based on $\operatorname{sl}(2, \mathbb{C})$ connections using the Haar measure (similar as we have done for $S U(2)$ for a fixed graph), its corresponding flux operator $\hat{B}_{I J}(S)$ becomes a linear combination of right invariant vector fields $R^{I J}$ on $S L(2, \mathbb{C})$. Now the simplicity constraint becomes the condition that the second Casimir operator $R^{I J} R^{K L} \epsilon_{I J K L}$ vanishes. However, on irreducible representations this operator is diagonal with eigenvalues $n \rho / 4$. While this is a strong motivation, it is certainly not sufficient justification for this way of implementing the simplicity constraint in the path integral because it is not clear how this is related to integrating over $\Phi$.

In any case, if one does this then one arrives at (some version of) the Lorentzian Barrett - Crane model [59]. Surprisingly, for a large class of triangulations $\tau$ the amplitudes

$$
K_{P}^{\tau}\left(\Sigma_{t_{1}}, \Sigma_{t_{2}}\right):=\left[\int[D \omega D B] e^{i S_{B F}[\omega, B]}\right]_{\mid \text {simplereps. }}
$$

actually converge although one integrates over a non-compact group! This is a non-trivial result [60. The path integral is then over all possible representations that label the faces of a spin foam and the boundary conditions keep the representations on the boundary graphs, that is, spin networks fixed $(S L(2, \mathbb{C})$ reduces to the $S U(2)$ on the boundary). This also answers the question of what the boundary conditions should be.

There is still an open issue, namely how one should get rid of the regulator (or triangulation) dependence. Since BF - theory is a topological QFT, the amplitudes are automatically triangulation independent, however, this is certainly not the case with GR. One possibility is to sum over triangulations and a concrete proposal of how to weigh the contributions from different triangulations comes from the so-called field theory formulation of the theory [61]. Here one reformulates the BF 
- theory path integral as the path integral for a scalar field on a group manifold which in this case is a certain power of $S L(2, \mathbb{C})$. The action for that scalar field has a free piece and an interaction piece and performing the perturbation theory (Feynman graphs !) for that field theory is equivalent to the sum over BF - theory amplitudes for all triangulated manifolds with precisely defined weights. This idea can be straightforwardly applied also to our context where the restriction to simple representations is realized by imposing corresponding restrictions (projections) on the scalar field.

Summarizing, spin foam models are a serious attempt to arrive at a covariant formulation of QGR but many issues are still unsettled, e.g.:

1)

There is no clean equivalence with the Hamiltonian formulation as we have seen. Without that it is unlcear how to interpret the spin foam model amplitude and whether it has the correct classical limit. In order to make progress on the issue of the classical limit, model independent techniques for constructing "causal spin foams" [62] with a built in notion of quantum causality and renormalization methods [63], which should allow in principle the derivation of a low energy effective action, have been developed.

2)

The physical correctness of the Barrett - Crane model is unclear. This is emphasized by recent results within the Euclidean formulation [64] which suggest that the classical limit is far off GR since the amplitudes are dominated by spin values close to zero. This was to be expected because in the definition of the Barrett - Crane model there is a certain flexibility concerning the choice of the measure that replaces [ $D \omega D B]$ at the triangulated level and the result [64] indicates that one must gain more control on that choice.

3)

It is not even clear that these models are four - dimensionally covariant: One usually defines that the amplitudes for a fixed triangulation are the same for any four - diffeomorphic triangulation. However, recent results [65] show that this natural definition could result nevertheless in anomalies. This problem is again related to the choice of the measure just mentioned.

Thus, substantially more work is required in order to fill in the present gaps but the results already obtained are very promising indeed.

\section{III.4 Quantum Black Holes}

\section{III.4.1 Isolated Horizons}

Any theory of quantum gravity must face the question whether it can reproduce the celebrated result due to Bekenstein and Hawking [66] that a black hole in a spacetime $(M, g)$ should account for a quantum statisical entropy given by

$$
S_{B H}=\frac{\operatorname{Ar}(H)}{4 \ell_{P}^{2}}
$$

where $H$ denotes the two-dimensional event horizon of the blak hole. This result was obtained within the framework of QFT on Curved SpaceTimes (CST) and should therefore be valid in a semiclassical regime in which quantum fluctuations of the gravitational field are neglible (large black holes). The most important question from the point of view of a microscopical theory of quantum gravity is, what are the microscopical degrees of freedom that give rise to that entropy. In particular, how can 
it be within a quantum field theory with an infinite number of degrees of freedom, that this entropy, presumably a measure for our lack of information of what happens behind the horizon, comes out finite.

In [67] the authors performed a bold computation: For any surface $S$ and any positive number $A_{0}$ they asked the question how many SNW states there are in QGR such that the area operators eigenvalues lie within the interval $\left[A_{0}-\ell_{P}^{2}, A_{0}+\ell_{P}^{2}\right]$. This answer is certainly infinite because a SNW can intersect $S$ in an uncountably infinite number of different positions without changing the eigenvalues. This divergence can be made less severe by moding out by spatial diffeomorphisms which we can use to map these different SNW onto each other in the vicinity of the surface. However, since there are still an infinite number of non-spatially difeomorphic states which look the same in the vicinity of the surface but different away from it, the answer is still divergent. Therefore, one has to argue that one must not count information off the surface, maybe invoking the Hamiltonian constraint or using the information that $S=H$ is not an arbitrary surface but actually the horizon of a black hole. Given this assumption, the result of the, actually quite simple counting problem came rather close to ([I.4.1.1) with the correct factor of $1 / 4$.

Thus the task left is to justify the assumptions made and to make the entropy counting water - tight by invoking the information that $H$ is a black hole horizon. The outcome of this analysis created a whole industry of its own, known under the name "isolated horizons", which to large part is a beautiful new chapter in classical GR. In what follows we will focus only on a tiny fraction of the framework, mostly concentrating on the ingredients essential for the quantum formulation. For reviews see 68 which also contain a complete list of references on the more classical aspects of this programme, the pivotal papers concerning the quantum applications are [69.

By definition, an event horizon is the external boundary of the part of $M$ that does not lie in the past of null future infinity $J^{+}$in a Penrose diagramme. From an operational point of view, this definition makes little sense because in order to determine whether a candidate is an event horizon, one must know the whole spacetime $(M, g)$ which is never possible by measurements which are necessarily loacal in spacetime (what looks like an eternal black hole now could capture some dust later and the horizon would change its location). Thus one looks for some local substitute of the notion of an event horizon which captures the idea that the black hole has come to some equilibrium state at least for some amount of time. This is roughly what an isolated horizon $\Delta$ is, illustrated in figure 14. More in technical details we have the following.

\section{Definition III.4.1.}

A part $\Delta$ of the boundary $\partial M$ of a spacetime $(M, g)$ is called an isolated horizon, provided that 1) $\Delta \equiv \mathbb{R} \times S^{2}$ is a null hypersurface and has zero shear and expansion $\mathbb{\square}$.

2) The field equations and matter energy conditions hold at $\Delta$.

3) $g$ is Lie derived by the null generator $l$ of $\Delta$ at $\Delta$.

The canonical formulation of a field theory on a manifold $M$ with boundary $\Delta$ must involve boundary conditions at $\Delta$ in order that the variation principle be well-defined (the action must be functionally differentiable). Such boundary conditions usually give birth to boundary degrees of freedom [70] which would normally be absent but now come into being because (part of the) gauge transformations are forced to become trivial at $\Delta$. In the present situation what happens is that the

\footnotetext{
${ }^{11}$ Recall the notions of shear, expansion and twist of a congruence of vector fields in connection with Raychaudhuri's equation.
} 


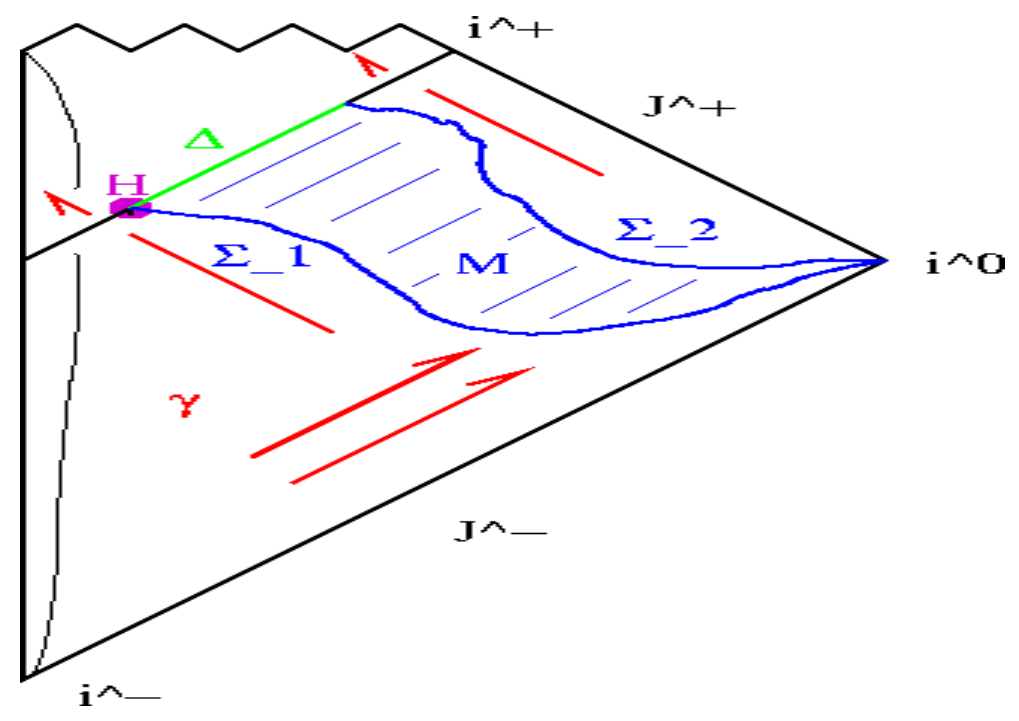

Figure 14: An isolated horizon $\Delta$ boundary of a piece $M$ (shaded) of spacetime also bounded by spacelike hypersurfaces $\Sigma_{1}, \Sigma_{2}$. Radiation $\gamma$ may enter or leave $M$ and propagate into the singularity before or after the isolated horizon has formed but must not cross $\Delta$. An intersection of a spacelike hypersurface $\Sigma$ with $\Delta$ is denoted by $H$ which has the topology of a sphere.

boundary term is actually a $U(1)$ Chern-Simons action ${ }^{\mathbb{T Z}}$

$$
S_{C S}=\frac{A_{0}}{\pi \beta} \int_{\Delta} W \wedge d W=\int_{\mathbb{R}} d t \int_{H} d^{2} y \epsilon^{I J}\left[\dot{W}_{I} W_{J}+W_{t}(d W)_{I J}\right]
$$

where $W$ is a $U(1)$ connection one form and $H=S^{2}=\Sigma \cap \Delta$ is a sphere. The relation between the bulk fields $A_{a}^{j}, E_{j}^{a}$ and the boundary fields $W_{I}, I=1,2$ is given by

$$
X_{H}^{*} A^{j}=W r^{j} \text { and }\left[X_{H}^{*}(* E)_{j}\right] r_{j}=-\frac{A_{0}}{2 \pi \beta} d W
$$

where $X_{H}: H \rightarrow \Sigma$ is the embedding of the boundary $H$ of $\Sigma$ into $\Sigma$ and $r^{j}$ is an arbitrary but fixed unit vector in $s u(2)$ which is to be preserved under $S U(2)$ gauge transformations at $\Delta$ and therefore reduces $S U(2)$ to $U(1)$. The number $A_{0}$ is the area of $H$ as measured by $g$ which turns out to be a constant of the motion as a consequence of the field equations. The existence of $r^{j}$ is a consequence of definition ([II.4.1) and $* E$ is the natural metric independent two - form dual to $E$.

\section{III.4.2 Entropy Counting}

One now has to quantize the system. This consists of several steps whose details are complicated and which we will only sketch in what follows.

i) Kinematical Hilbert Space

The bulk and boundary degrees of freedom are independent of each other, therefore we choose $\mathcal{H}_{\text {kin }}=\mathcal{H}_{\text {kin }}^{\Sigma} \otimes \mathcal{H}_{\text {kin }}^{H}$ where both spaces are of the form $L_{2}\left(\overline{\mathcal{A}}, d \mu_{0}\right)$ just that the first factor is for an $S U(2)$ bundle over $\Sigma$ while the second is for an $U(1)$ bundle over $H$.

\footnotetext{
${ }^{12}$ It was observed first in [71] that general relativity in terms of connection variables and in the presence of boundaries leads to Chern - Simons boundary terms.
} 
ii) Quantum Boundary conditions

Equation ([II.4.1.3) implies, in particular, that in quantum theory we must have schematically

$$
\left.\left[\left[X_{H}^{*(* E)}\right]_{j}\right] r_{j}\right] \otimes \operatorname{id}_{\mathcal{H}_{k i n}^{H}}=\operatorname{id}_{\mathcal{H}_{k i n}^{\Sigma}} \otimes\left[-\frac{A_{0}}{2 \pi \beta} \widehat{d W}\right]
$$

Now we have seen in the bulk theory that we have discussed in great detail throughout this review, that $* E$ is an operator valued distribution which must be smeared by two - surfaces in order to arrive at the well-defined electric fluxes. Since (III.4.2.1) is evaluated at $H$, this flux operator will non-trivially act only on SNWF's $T_{s}$ which live in the bulk but intersect $H$ in punctures $p \in H \cap \gamma(s)$. Now the distributional character of the electric fluxes implies that the left hand side of (III.4.2.1) is non-vanishing only at those punctures. Thus the curvature of $W$ is flat everywhere except for the punctures where it is distributional.

Consider now SNWF's $T_{s}$ of the bulk theory and those of the boundary theory $T_{c}^{\prime}$. Then $\left.\left[X_{H}^{*}(* E)_{j}\right] r_{j}\right]$ acts on $T_{s}$ like the $z$-component of the angular momentum operator and will have distributional eigenvalues proportional to the magnetic quantum numbers $m_{e}$ of the edges with punctures $p=e \cap H$ and spin $j_{e}$ where $m_{e} \in\left\{-j_{e},-j_{e}+1, . ., j_{e}\right\}$.

iii) Implementation of Quantum Dynamics at $\Delta$

It turns out that $\left.X_{H}^{*} \widehat{(* E)} j\right] r_{j}$ and $\widehat{d W}$ are the generators of residual $S U(2)$ gauge transformations close to $X_{H}(H)$ and of $U(1)$ on $H$ respectively. Now these residual $S U(2)$ transformations are frozen to $U(1)$ transformations by $r_{j}$ and the most general situation in order for a state to be gauge invariant is that these residual $S U(2)$ transformations of the bulk theory and the $U(1)$ transformations of the boundary theory precisely cancel each other. It turns out that this cancellation condition is precisely given by the quantum boundary condition (III.4.2.1). Thus the states that solve the Gauss constraint are linear combinations of states of the form $T_{s} \otimes T_{c}^{\prime}$ where the boundary data of these states are punctures $p \in \mathcal{P}$ where $p \in \gamma(s) \cap H$, the spins $j_{p}=j_{e_{p}}$ of edges $e \in E(\gamma(s))$ with $e_{p} \cap H=p$ and their magnetic quantum numbers $m_{p}=m_{e_{p}}$. However, due to the specific features of the geometrical quantization of Chern-Simons theories [72 the $m_{p}$ cannot be specified freely, they have to satisfy the constraint

$$
\sum_{p \in \mathcal{P}} 2 m_{p}=0 \bmod k, \quad k=\frac{A_{0}}{4 \pi \ell_{P}^{2}}
$$

where $k$ is called the level of a Chern Simons theory which is constrained to be an integer due to Weil's quantization obstruction cocycle criterion of geometric quantization [30] and comes about as follows: The $T_{c}^{\prime}$ are actually fixed to be $\Theta$-functions of level $k$ labelled by integers $a_{p}$ which satisfy the gauge invariance condition

$$
2 m_{p}=-a_{p} \bmod k, \sum_{p} a_{p}=0 \bmod k
$$

Next, the spatial diffeomorphism constraint of the bulk theory tells us that the position of the punctures on $H$ are not important, important is only their number.

Finally, one of the boundary conditions at $\Delta$ implies that the lapse becomes trivial $N=0$ at $H$ if $\hat{C}(N)$ is to generate an infinitesimal time reparameterization [?] Thus, luckily we can

${ }^{13}$ This does not mean that the lapse of a classical isolated horizon solution must vanish at $S$, rather there is a subtle difference between gauge motions and symmetries for field theories with boundaries $[70]$ where in this case symmetries map solutions to gauge inequivalent or equivalent ones respectively, if $N_{\mid H} \neq 0$ or $N_{\mid H}=0$ respectively. 


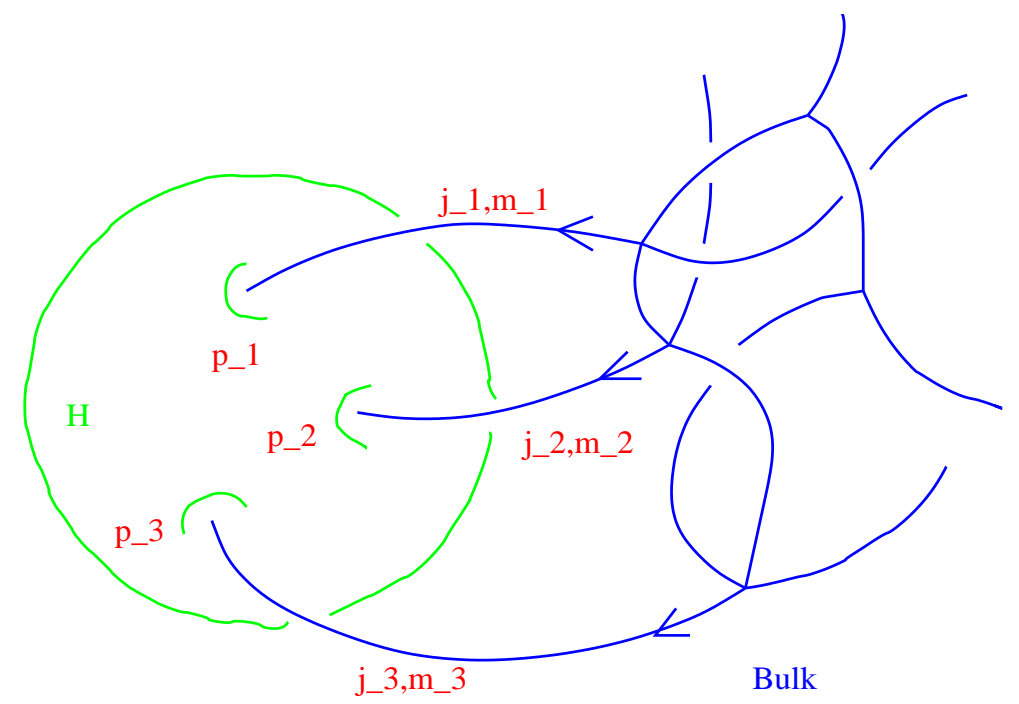

Figure 15: Punctures, spins, magnetic quantum numbers and entropy counting. Only the relevant boundary data are shown, the bulk information is traced over.

escape the open issues with the Hamiltonian constraint as far as the quantum dynamics at $H$ is concerned.

We can now come to the issue of entropy counting. First of all we notice that $\operatorname{Ar}(H)$ is a Dirac observable because $H$ is invariant under $\operatorname{Diff}(H)$ and $N=0$ at $H$. Given $n$ punctures with spins $j_{l}, l=1, . ., N$ the area eigenvalue for $H$ is

$$
\lambda(n, \vec{j})=8 \pi \ell_{P}^{2} \beta \sum_{n=1}^{n} \sqrt{j_{l}\left(j_{l}+1\right)}
$$

Now the physical Hilbert space is of the form

$$
\mathcal{H}_{\text {phys }}=\bigoplus_{n, \vec{j}, \vec{m}, \vec{a}={ }_{k}-2 \vec{m}} \mathcal{H}_{n, \vec{j}, \vec{m}}^{B} \otimes \mathcal{H}_{n, \vec{j}, \vec{m}}^{B H} \otimes \mathcal{H}_{n, \vec{a}}^{H}
$$

where $\mathcal{H}_{n, \vec{j}, \vec{m}}^{B H}$ describes bulk degrees of freedom at $H$ corresponding to the black hole (finite dimensional), $\mathcal{H}_{n, \vec{j}, \vec{m}}^{B}$ describes bulk degrees of freedom away from $H$ and finally $\mathcal{H}_{n, \vec{a}}^{H}$ describes Chern Simons degrees of freedom which are completetly fixed in tems of $\vec{m}$ due to reasons of gauge invariance (III.4.2.3). The situation is illustrated in figure 15. Let $\delta>0$ and let $S_{A_{0}, \delta}$ be the set of eigenstates $\psi_{n, \vec{j}, \vec{m}} \in \mathcal{H}_{n, \vec{j}, \vec{m}}^{B H}$ of the area operator such that the eigenvalue lies in the interval $\left[A_{0}-\delta, A_{0}+\delta\right]$ and $N_{A_{0}, \delta}$ their number. Define the density matrix

$$
\hat{\rho}_{B H}=\operatorname{id}_{B} \otimes\left[\frac{1}{N} \sum_{A_{0}, \delta} \sum_{\psi \in S_{A_{0}, \delta}}|\psi><\psi|\right] \otimes \operatorname{id}_{H}
$$

The quantum statistical entropy from this microcanonical ensemble is given by

$$
S_{B H}=-\operatorname{Tr}\left(\hat{\rho}_{B H} \ln \left(\hat{\rho}_{B H}\right)\right)=\ln \left(N_{A_{0}, \delta}\right)
$$

Thus we just need to count states and the answer will be finite because the area operator has an area gap. 


\section{Exercise III.4.1.}

Estimate $N_{A_{0}, \delta}$ from above and below taking into account the constraint (111.4.2.9) and that $k$ is an integer (purely combinatorical problem!).

The result of the counting problem is that $S_{B H}$ is indeed given by (III.4.1.1) to leading order in $A_{0}$ (there are logarithmic corrections) for $\delta \approx \ell_{P}^{2}$ provided that

$$
\beta=\frac{\ln (2)}{\pi \sqrt{3}}
$$

Here the numbers $\ln (2), \sqrt{3}$ comes from the fact that the configurations with lowest spin $j_{l}=1 / 2$ make the dominant contribution to the entropy with eigenvalue $\propto \beta n \sqrt{3} \approx A_{0}$ and number of states given by $N_{A_{0}, \delta} \approx 2^{n}$ that is, two Boolean degrees of freedom per puncture [73]. This provides an explicit explanation for the origin of the entropy. Now fixing $\beta$ at the value ([II.4.2.8) would make little sense would it be different for different types of black hole (that is, in presence of different matter, charges, rotation, other hair,..). However, this is not the case !

In summary, the analysis sketched above provides a self-contained derivation of $S_{B H}$ within QGR. The result is highly non-trivial because it was not to be expected from the outset that Loop Quantum Gravity, classical GR and Chern Simons theory would interact in such a harmonic way as to provide the expected result: Chern - Simons theory is very different from QGR and still they have an interface at $H$. The result applies to astrophysically interesting balck holes of the Schwarzschild type and does not require supersymmetry. Nevertheless, the calculation still has a semiclassical input because the presence of the isolated horizon is fed in at the classical level already. It would be more satisfactory to have a quantum definition of an (isolated) horizon but this is a hard task and left for future research. Another unsolved problem then is the calculation of the Hawking effect from first principles.

\section{III.5 Semiclassical Analysis}

\section{III.5.1 The Complexifier Machinery for Generating Coherent States}

Let us first specify what we mean by semiclassical states.

\section{Definition III.5.1.}

Let be given a phase space $\mathcal{M},\{.,$.$\} with preferred Poisson subalgebra \mathcal{O}$ of $C^{\infty}(\mathcal{M})$ and a Hilbert space $\mathcal{H}$, [.,.] together with an operator subalgebra $\hat{\mathcal{O}}$ of $\mathcal{L}(\mathcal{H})$. The triple $\mathcal{M},\{.,\},. \mathcal{O}$ is said to be a classical limit of the triple $\mathcal{H},[.,],. \hat{\mathcal{O}}$ provided that there exists an (over) complete set of states $\left\{\psi_{m}\right\}_{m \in \mathcal{M}}$ such that for all $O, O^{\prime} \in \mathcal{O}$ the infinitesimal Ehrenfest property

$$
\begin{aligned}
& \left|\frac{<\hat{O}>_{m}}{O(m)}-1\right| \ll 1 \\
& \left|\frac{<\left[\hat{O}, \hat{O}^{\prime}\right]>_{m}}{i \hbar\left\{O, O^{\prime}\right\}(m)}-1\right| \ll 1
\end{aligned}
$$

and the small fluctuation property

$$
\left|\frac{<\hat{O}^{2}>_{m}}{<\hat{O}>_{m}^{2}}-1\right| \ll 1
$$


holds at generiq points in $\mathcal{M}$. Here $<.>_{m}:=<\psi_{m}, . \psi_{m}>/\left\|\psi_{m}\right\|^{2}$ denotes the expectation value functional.

For systems with constraints, strictly speaking, semiclassical states should be physical states, that is, those that solve the constraints because we are not interested in approximating gauge degrees of freedom but only physical observables. Only then are the predictions ( $\hbar$ corrections to the classical limit) of the theory reliable. In the present situation with QGR, however, we are more interested in constructing kinematical semiclassical states for the following reason: As we have shown, the status of the physical correctness of the Hamiltonian constraint operator $\hat{C}$ is unsettled. We would therefore like to test whether it has the correct classical limit. This test is obviously meaningless on states which the Hamiltonian constraint annihilates anyway. For the same reason it also does not make sense to construct semiclassical states which are at least spatially diffeomorphism invariant because the Hamiltonian constraint does not leave this space invariant.

The key question then is how to construct semiclassical states. Fortunately, for phase spaces which have a cotangent bundle structure as is the case with QGR, a rather general construction guideline is available [74], the so-called Complexifier Method, which we will now sketch:

Let $(\mathcal{M},\{.,\}$.$) be a phase space with (strong) symplectic structure \{.,$.$\} (notice that \mathcal{M}$ is allowed to be infinite dimensional). We will assume that $\mathcal{M}=T^{*} \mathcal{C}$ is a cotangent bundle. Let us then choose a real polarization of $\mathcal{M}$, that is, a real Lagrangean submanifold $\mathcal{C}$ which will play the role of our configuration space. Then a loose definition of a complexifier is as follows:

Definition III.5.2. A complexifier is a positive definite function $\mathrm{m} C$ on $\mathcal{M}$ with the dimension of an action, which is smooth a.e. (with respect to the Liouville measure induced from $\{.,$.$\} ) and$ whose Hamiltonian vector field is everywhere non-vanishing on $\mathcal{C}$. Moreover, for each point $q \in \mathcal{C}$ the function $p \mapsto C_{q}(p)=C(q, p)$ grows stronger than linearly with $\|p\|_{q}$ where $p$ is a local momentum coordinate and $\|.\|_{q}$ is a suitable norm on $T_{q}^{*}(\mathcal{C})$.

In the course of our discussion we will motivate all of these requirements.

The reason for the name complexifier is that $C$ enables us to generate a complex polarization of $\mathcal{M}$ from $\mathcal{C}$ as follows: If we denote by $q$ local coordinates of $\mathcal{C}$ (we do not display any discrete or continuous labels but we assume that local fields have been properly smeared with test functions) then

$$
z(m):=\sum_{n=0}^{\infty} \frac{i^{n}}{n !}\{q, C\}_{(n)}(m)
$$

define local complex coordinates of $\mathcal{M}$ provided we can invert $z, \bar{z}$ for $m:=(q, p)$ where $p$ are the fibre (momentum) coordinates of $\mathcal{M}$. This is granted at least locally by definition 111.5 .2 . Here the multiple Poisson bracket is inductively defined by $\{q, C\}_{(0)}=q,\{q, C\}_{(n+1)}=\left\{\{q, C\}_{(n)}, C\right\}$ and makes sense due to the required smoothness. What is interesting about ([II.5.1.3) is that it implies the following bracket structure

$$
\{z, z\}=\{\bar{z}, \bar{z}\}=0
$$

while $\{z, \bar{z}\}$ is necessarily non-vanishing. The reason for this is that (III.5.1.3) may be written in the more compact form

$$
z=e^{-i \mathcal{L}_{\chi_{C}}} q=\left(\left[\varphi_{\chi_{C}}^{t}\right]^{*} q\right)_{t=-i}
$$

\footnotetext{
${ }^{14}$ The set of points where ([II.5.1.1), ([II.5.1.2) are violated should have small Liouville measure.

${ }^{15}$ For the rest of this section $C$ will denote a complexifier function and not the Hamiltonian constraint.
} 
where $\chi_{C}$ denotes the Hamiltonian vector field of $C, \mathcal{L}$ denotes the Lie derivative and $\varphi_{\chi_{C}}^{t}$ is the one - parameter family of canonical transformations generated by $\chi_{C}$. Formula ([II.5.1.5) displays the transformation ([III.5.1.3) as the analytic extension to imaginary values of the one parameter family of diffeomorphisms generated by $\chi_{C}$ and since the flow generated by Hamiltonian vector fields leaves Poisson brackets invariant, ([II.5.1.4) follows from the definition of a Lagrangean submanifold. The fact that we have continued to the negative imaginary axis rather than the positive one is important in what follows and has to do with the required positivity of $C$.

The importance of this observation is that either of $z, \bar{z}$ are coordinates of a Lagrangean submanifold of the complexification $\mathcal{M}^{\mathbb{C}}$, i.e. a complex polarization and thus may serve to define a Bargmann-Segal representation of the quantum theory (wave functions are holomorphic functions of $z)$. The diffeomorphism $\mathcal{M} \rightarrow \mathcal{C}^{\mathbb{C}} ; m \mapsto z(m)$ shows that we may think of $\mathcal{M}$ either as a symplectic manifold or as a complex manifold (complexification of the configuration space). Indeed, the polarization is usually a positive Kähler polarization with respect to the natural $\{.,$.$\} -compatible complex$ structure on a cotangent bundle defined by local Darboux coordinates, if we choose the complexifier to be a function of $p$ only. These facts make the associated Segal-Bargmann representation especially attractive.

We now apply the rules of canonical quantization: a suitable Poisson algebra $\mathcal{O}$ of functions $O$ on $\mathcal{M}$ is promoted to an algebra $\hat{\mathcal{O}}$ of operators $\hat{O}$ on a Hilbert space $\mathcal{H}$ subject to the condition that Poisson brackets turn into commutators divided by $i \hbar$ and that reality conditions are reflected as adjointness relations, that is,

$$
\left[\hat{O}, \hat{O}^{\prime}\right]=i \hbar\left\{\widehat{O, O^{\prime}}\right\}+o(\hbar), \quad \hat{O}^{\dagger}=\hat{\bar{O}}+o(\hbar)
$$

where quantum corrections are allowed (and in principle unavoidable except if we restrict $\mathcal{O}$, say to functions linear in momenta). We will assume that the Hilbert space can be represented as a space of square integrable functions on (a distributional extension $\overline{\mathcal{C}}$ of $) \mathcal{C}$ with respect to a positive, faithful probability measure $\mu$, that is, $\mathcal{H}=L_{2}(\overline{\mathcal{C}}, d \mu)$ as it is motivated by the real polarization.

The fact that $C$ is positive motivates to quantize it in such a way that it becomes a self-adjoint, positive definite operator. We will assume this to be the case in what follows. Applying then the quantization rules to the functions $z$ in (III.5.1.3) we arrive at

$$
\hat{z}=\sum_{n=0}^{\infty} \frac{i^{n}}{n^{!}} \frac{[\hat{q}, \hat{C}]_{(n)}}{(i \hbar)^{n}}=e^{-\hat{C} / \hbar} \hat{q} e^{\hat{C} / \hbar}
$$

The appearence of $1 / \hbar$ in ([II.5.1.7) justifies the requirement for $C / \hbar$ to be dimensionless in definition III.5.2. We will call $\hat{z}$ annihilation operator for reasons that will become obvious in a moment.

Let now $q \mapsto \delta_{q^{\prime}}(q)$ be the $\delta$-distribution with respect to $\mu$ with support at $q=q^{\prime}$. (More in mathematical terms, consider the complex probability measure, denoted as $\delta_{q^{\prime}} d \mu$, which is defined by $\int \delta_{q^{\prime}} d \mu f=f\left(q^{\prime}\right)$ for measurable $\left.f\right)$. Notice that since $C$ is non-negative and necessarily depends non-trivially on momenta (which will turn into (functional) derivative operators in the quantum theory), the operator $e^{-\hat{C} / \hbar}$ is a smoothening operator. Therefore, although $\delta_{q^{\prime}}$ is certainly not square integrable, the complex measure (which is probability if $\hat{C} \cdot 1=0$ )

$$
\psi_{q^{\prime}}:=e^{-\hat{C} / \hbar} \delta_{q^{\prime}}
$$

has a chance to be an element of $\mathcal{H}$. Whether or not it does depends on the details of $\mathcal{M},\{.,\},$.$C .$ For instance, if $C$ as a function of $p$ at fixed $q$ has flat directions, then the smoothening effect of 
$e^{-\hat{C} / \hbar}$ may be insufficient, so in order to avoid this we required that $C$ is positive definite and not merely non-negative. If $C$ would be indefinite, then ([II.5.1.8) has no chance to make sense as an $L_{2}$ function.

We will see in a moment that ([11.5.1.8) qualifies as a candidate coherent state if we are able to analytically extend ([II.5.1.8) to complex values $z$ of $q^{\prime}$ where the label $z$ in $\psi_{z}$ will play the role of the point in $\mathcal{M}$ at which the coherent state is peaked. In order that this is possible (and in order that the extended function is still square integrable), ([II.5.1.8) should be entire analytic. Now $\delta_{q^{\prime}}(q)$ roughly has an integral kernel of the form $e^{i\left(k,\left(q-q^{\prime}\right)\right)}$ (with some pairing $<., .>$ between tangential and cotangential vectors) which is analytic in $q^{\prime}$ but the integral over $k$, after applying $e^{-\hat{C} / \hbar}$, will produce an entire analytic function only if there is a damping factor which decreases faster than exponentially. This provides the intuitive explanation for the growth requirement in definition [11.5.2. Notice that the $\psi_{z}$ are not necessarily normalized.

Let us then assume that

$$
q \mapsto \psi_{m}(q):=\left[\psi_{q^{\prime}}(q)\right]_{q^{\prime} \rightarrow z(m)}=\left[e^{-\hat{C} / \hbar} \delta_{q^{\prime}}(q)\right]_{q^{\prime} \rightarrow z(m)}
$$

is an entire $L_{2}$ function. Then $\psi_{m}$ is automatically an eigenfunction of the annihilation operator $\hat{z}$ with eigenvalue $z$ since

$$
\hat{z} \psi_{m}=\left[e^{-\hat{C} / \hbar} \hat{q} \delta_{q^{\prime}}\right]_{q^{\prime} \rightarrow z(m)}=\left[q^{\prime} e^{-\hat{C} / \hbar} \delta_{q^{\prime}}\right]_{q^{\prime} \rightarrow z(m)}=z(m) \psi_{m}
$$

where in the second step we used that the delta distribution is a generalized eigenfunction of the operator $\hat{q}$. But to be an eigenfunction of an annihilation operator is one of the accepted definitions of coherent states!

Next, let us verify that $\psi_{m}$ indeed has a chance to be peaked at $m$. To see this, let us consider the self-adjoint (modulo domain questions) combinations

$$
\hat{x}:=\frac{\hat{z}+\hat{z}^{\dagger}}{2}, \hat{y}:=\frac{\hat{z}-\hat{z}^{\dagger}}{2 i}
$$

whose classical analogs provide real coordinates for $\mathcal{M}$. Then we have automatically from ([II.5.1.10)

$$
<\hat{x}>_{m}:=\frac{<\psi_{m}, \hat{x} \psi_{m}>}{\left\|\psi_{m}\right\|^{2}}=\frac{z(m)+\bar{z}(m)}{2}=: x(m)
$$

and similar for $y$. Equation (III.5.1.12) tells us that the operator $\hat{z}$ should really correspond to the function $m \mapsto z(m), m \in \mathcal{M}$.

Now we compute by similar methods that

$$
<[\delta \hat{x}]^{2}>_{m}:=\frac{<\psi_{m},\left[\hat{x}-<\hat{x}>_{m}\right]^{2} \psi_{m}>}{\left\|\psi_{m}\right\|^{2}}=<[\delta \hat{y}]^{2}>_{m}=\frac{1}{2}\left|<[\hat{x}, \hat{y}]>_{m}\right|
$$

so that the $\psi_{m}$ are automatically minimal uncertainty states for $\hat{x}, \hat{y}$, moreover the fluctuations are unquenched (equal each other). This is the second motivation for calling the $\psi_{m}$ coherent states. Certainly one should not only check that the fluctuations are minimal but also that they are small as compared to the expectation value, at least at generic points of the phase space, in order that the quantum errors are small.

The infinitesimal Ehrenfest property

$$
\frac{<[\hat{x}, \hat{y}]>_{z}}{i \hbar}=\{x, y\}(m)+O(\hbar)
$$


follows if we have properly implemented the canonical commutation relations and adjointness relations. The size of the correction, however, does not follow from these general considerations but the minimal uncertainty property makes small corrections plausible. Condition (III.5.1.14) supplies information about how well the symplectic structure is reproduced in the quantum theory.

For the same reason one expects that the peakedness property

$$
\mid \frac{<\psi_{m}, \psi_{m^{\prime}}>\left.\right|^{2}}{\left\|\psi_{m}||^{2}\right\| \psi_{m^{\prime}} \|^{2}} \approx \chi_{K_{m}}\left(m^{\prime}\right)
$$

holds, where $K_{m}$ is a phase cell with center $m$ and Liouville volume $\approx \sqrt{\left\langle[\delta \hat{x}]^{2}>_{m}<[\delta \hat{y}]^{2}>_{m}\right.}$ and $\chi$ denotes the characteristic function of a set.

Finally one wants coherent states to be overcomplete in order that every state in $\mathcal{H}$ can be expanded in terms of them. This has to be checked on a case by case analysis but the fact that our complexifier coherent states are for real $z$ nothing else than regularized $\delta$ distributions which in turn provide a (generalized) basis makes this property plausible to hold.

\section{Exercise III.5.1.}

Consider the phase space: $\mathcal{M}=T^{*} \mathbb{R}=\mathbb{R}^{2}$ with standard Poisson brackets $\{q, q\}=\{p, p\}=$ $0,\{p, q\}=1$ and configuration space $\mathcal{C}=\mathbb{R}$. Consider the complexifier $C=p^{2} /(2 \sigma)$ where $\sigma$ is a dimensionful constant such that $C / \hbar$ is dimensionfree. Check that it meets all the requirements of definition III.5.9 and perform the coherent state construction displayed above.

Hint:

Up to a phase, the resulting, normalized coherent states are the usual ones for the harmonic oscillator with Hamiltonian $H=\left(p^{2} / m+m \omega^{2} q^{2}\right) / 2$ with $\sigma=m \omega$. Verify that the states $\psi_{m}$ are Gaussian peaked in the configuration representation with width $\sqrt{\hbar / \sigma}$ around $q=q_{0}$ and in the momentum representation around $p=p_{0}$ with width $\sqrt{\hbar \sigma}$ where $m=\left(p_{0}, q_{0}\right)$.

As it has become clear from the discussion, the complexifier method gives a rough guideline, but no algorithm, in order to arrive at a satisfactory family of coherent states, there are things to be checked on a case by case basis. On the other hand, what is nice is that given only one input, namely the complexifier $C$, it is possible to arrive at a definite and constructive framework for a semiclassical analysis. It is important to know what the classical limit of $\hat{C}$ is, otherwise, if we have just an abstract operator $\hat{C}$ then the map $m \mapsto z(m)$ is unknown and an interprtation of the states in terms of $\mathcal{M}$ is lost.

\section{III.5.2 Application to QGR}

Let us now apply these ideas to QGR. Usually the choice of $C$ is stronly motivated by a Hamiltonian, but in QGR we have none. Therefore, at the moment the best we can do is to play with various proposals for $\hat{C}$ and to explore the properties of the resulting states. For the simplest choice of $\hat{C}$ 755 those properties have been worked out more or less completely and we will briefly describe them below.

The operator $\hat{C}$ is defined by its action on cylindrical functions $f=p_{\gamma}^{*} f_{\gamma}$ by

$$
\frac{\hat{C}}{\hbar} f=-p_{\gamma}^{*}\left[\frac{1}{2}\left[\sum_{e \in E(\gamma)} l(e)\left[R_{e}^{j} / 2\right]^{2}\right] f_{\gamma}\right]=: p_{\gamma}^{*}\left[\hat{C}_{\gamma} f_{\gamma}\right]
$$

where the positive numbers $l(e)$ satisfy $l\left(e \circ e^{\prime}\right)=l(e)+l\left(e^{\prime}\right)$ and $l\left(e^{-1}\right)=l(e)$ and $R_{e}^{j}$ are the usual right invariant vector fields. 


\section{Exercise III.5.2.}

Recall the definition of the maps $p_{\gamma^{\prime} \gamma}$ for $\gamma \prec \gamma^{\prime}$ from section II.1.9 and check that the $\hat{C}_{\gamma}$ are consistently defined, that is, $\hat{C}_{\gamma^{\prime}} \circ p_{\gamma^{\prime} \gamma}^{*}=p_{\gamma^{\prime} \gamma}^{*} \circ \hat{C}_{\gamma}$.

This choice is in analogy to the harmonic oscillator where the quantum complexifier is essentially the Laplacian $-(d / d x)^{2}$. The classical limit of (III.5.2.1) depends in detail on the function $l$ which is analogous to the parameter $\hbar / \sigma$ for the case of the harmonic oscillator. For instance [74], one can choose a) three families of foliations $s \mapsto H_{s}^{I}, I=1,2,3$ of $\sigma$ by two dimensional surfaces $H_{s}^{I}$ such that there is a bijection $\left(s^{1}, s^{2}, s^{3}\right) \mapsto x(\vec{s}):=\left[\cap_{I} H_{s^{I}}^{I}\right] \in \sigma$ and b) a partition $P_{s}^{I}$ of the $H_{s}^{I}$ into small surfaces $S$ and define

$$
C=\frac{1}{2 a^{2} \kappa} \int_{\mathbb{R}} d s \sum_{I=1}^{3} \sum_{S \in P_{s}^{I}}[\operatorname{Ar}(S)]^{2}
$$

where $\operatorname{Ar}(S)$ is again the area functional and $a$ is a dimensionful constant of dimension $\mathrm{cm}^{1}$. The function $l$ for this example is then roughly $l(e)=\frac{\left(\beta \ell_{P}\right)^{2}}{a^{2}} \int d s \sum_{I} \sum_{S \in P_{S}^{I}} \chi_{S}(e)$ where $\chi_{S}(e)=1$ if $S \cap e \neq \emptyset$ and vanishes otherwise.

The $\delta$-distribution with respect to the measure $\mu_{0}$ can be written as the sum over all SNW's (exercise !)

$$
\delta_{A^{\prime}}(A)=\sum_{s} T_{s}\left(A^{\prime}\right) \overline{T_{s}(A)}
$$

with resulting coherent states

$$
\psi_{A^{\mathbb{C}}}(A)=\sum_{s} e^{-\frac{1}{2} \sum_{e \in E(\gamma(s))} l(e) j_{e}\left(j_{e}+1\right)} T_{s}\left(A^{\mathbb{C}}\right) \overline{T_{s}(A)}
$$

where the $S L(2, \mathbb{C})$ connection $A^{\mathbb{C}}$ is defined by

$$
A^{\mathbb{C}}[A, E]=\sum_{n=0}^{\infty} \frac{i^{n}}{n !}\left(\{A, C\}_{(n)}\right)[A, E]
$$

Thus we see that in this case the symplectic manifold given as the cotangential bundle $\mathcal{M}=T^{*} \mathcal{A}$ over the space of $S U(2)$ connections is also naturally given as the complex manifold $\mathcal{A}^{\mathbb{C}}$ of $S L(2, \mathbb{C})$ connections. From the general discussion above it now follows that the classical interpretation of the annihilation operators

$$
\hat{A}^{\mathbb{C}}(e):=e^{-\hat{C} / \hbar} \hat{A}(e) e^{\hat{C} / \hbar}
$$

is simply the holonomy of the complex connection $A^{\mathbb{Q}}(e)$.

In order to study the semiclassical properties of these states we consider their cut-offs $\psi_{A^{\mathbb{Q}}, \gamma}$ for each graph $\gamma$ defined on cylindrical functions $f=p_{\gamma}^{*} f_{\gamma}$ by

$$
<\overline{\psi_{A^{\mathbb{T}}}}, f>_{k i n}=:<\overline{\psi_{A^{\mathbb{G}}, \gamma}}, f>_{k i n}
$$

Now, (if we work at the non-gauge invariant level,) one can check that

$$
\psi_{A^{\mathbb{G}}, \gamma}(A)=\prod_{e \in E(\gamma(s)} \psi_{A^{\mathbb{T}}(e)}^{l(e)}(A(e))
$$

\footnotetext{
${ }^{16}$ This formula gets exact in the limit of infinitely fine partition, at finite coarseness, it is an approximation to the exact, more complicated formula.
} 
where for any $g \in S L(2, \mathbb{C}), h \in S U(2)$ we have defined

$$
\psi_{g}^{t}(h):=\sum_{j}(2 j+1) e^{-t j(j+1) / 2} \chi_{j}\left(g h^{-1}\right)
$$

\section{Exercise III.5.3.}

Verify, using the Peter\&WWeyl theorem, that for $g \in S U(2)$ we have $\psi_{g}^{0}(h)=\delta_{g}(h)$, the $\delta$-distribution with respect to $L_{2}\left(S U(2), d \mu_{H}\right)$. Conclude that (M1.5.2.9) is just the analytic extension of the heat kernel $e^{-t \Delta / 2}$ where $\Delta$ is the Laplacian on $S U(2)$. Thus the states (III.5.2.9) are in complete analogy with those for the harmonic oscillator, just that $\mathbb{R}$ was replaced by $S U(2)$ and the complexification $\mathbb{C}$ of $\mathbb{R}$ by the complexification $S L(2, \mathbb{C})$ of $S U(2)$. In this form, coherent states on compact gauge groups were originally proposed by Hall [70].

The analysis of the semiclassical properties of the states $\psi_{A^{\mathbb{d}}, \gamma}$ on $\mathcal{H}_{k i n}$ can therefore be reduced to that of the states $\psi_{g}^{t}$ on $L_{2}\left(S U(2), d \mu_{H}\right)$. We state here without proof that the following properties could be proved [75]: I) Overcompleteness, II) expectation value property, III) Ehrenfest property, IV) peakedness in phase space, V) annihilation operator eigenstate property, VI) minimal uncertainty property and VII) small fluctuation property. Thus, these states have many of the desired properties that one requires from coherent states.

In the following graphic we display as an example the peakedness properties of the analog of (III.5.2.9) for the simpler case of the gauge group $U(1)$, the case of $S U(2)$ is similar but requires more plots because of the higher dimensionality of $S U(2)$. Thus $g_{0}=e^{p} h_{0} \in U(1)^{\mathbb{C}}=\mathbb{C}-\{0\}, p \in$ $\mathbb{R}, h_{0} \in U(1)$ and $u \in U(1)$ where we parameterize $u=e^{i \phi}, \phi \in[-\pi, \pi)$. Similarly, $g=e^{p_{1}} u, p_{1} \in \mathbb{R}$. We consider in figure 16 the peakedness in the configuration representation given by the probability amplitude

$$
u=e^{i \phi} \mapsto j_{g_{0}}^{t}(u)=\left|\psi_{g_{0}}^{t}(u)\right|^{2} /\left\|\psi_{g_{0}}^{t}\right\|^{2}
$$

at $h_{0}=1, p \in[-5,5]$. In figure 17 the phase space peakedness expressed by the overlap function

$$
g=e^{p_{1}} u \mapsto i^{t}\left(g, g_{0}\right)=\frac{\left|<\psi_{g}^{t}, \psi_{g_{0}}^{t}>\right|^{2}}{\left.\left\|\psi_{g}^{t}||^{2}\right\| \psi_{g_{0}}^{t}\right|^{2}}
$$

is shown at fixed $p=0, h_{0}=1$ for $p \in[-5,5], u \in U(1)$. We have made use of the fact (exercise !) that $\psi_{g_{0}}(u)$ and $\left\langle\psi_{g}, \psi_{g_{0}}>\right.$ respectively depend only on the combinations $g_{0} u^{-1}=e^{p_{0}} h u^{-1}$ and $\bar{g} g_{0}=e^{p+p_{1}} u^{-1} h_{0}$ respectively. Therefore, peakedness at $u=h_{0}$ or $g=g_{0}=e^{p} h_{0}$ respectively for any $h_{0}$ is equivalent to peakedness at $u=1$ or at $g=e^{p_{0}}$ respectively for $h_{0}=1$. Both plots are for the value $t=0.001$ and one clearly sees the peak width of $\sqrt{t} \approx 0.03$ when resolving those plots around the peak as in figure 18, which has a close to Gaussian shape just like the harmonic oscillator coherent states have. As a first modest application, these states have been used in order to analyze how one would obtain, at least in principle, the QFT's on CST's (Curved SpaceTime) limit from full QGR in 49. In particular, it was possible to perform a detailed calculation concerning the existence of Poincaré invariance violating dispersion relations of photon propagation within QGR which were discussed earlier at a more phenomenological level in the pioneering papers [77]: The idea is that the metric field is a collection of quantum operators which are not mutually commuting. Therefore it should be impossible to construct a state which is peaked on, say the Minkowski metric, and which is a simultaneous eigenstate of all the metric operator components, in other words, there should be no such thing as a Poincar/'e invariant state in full QGR $\square$, already because such an object should

\footnotetext{
${ }^{17}$ This seems to contradict the fact that we are even interested in four dimensionally diffeomorphism invariant states
} 


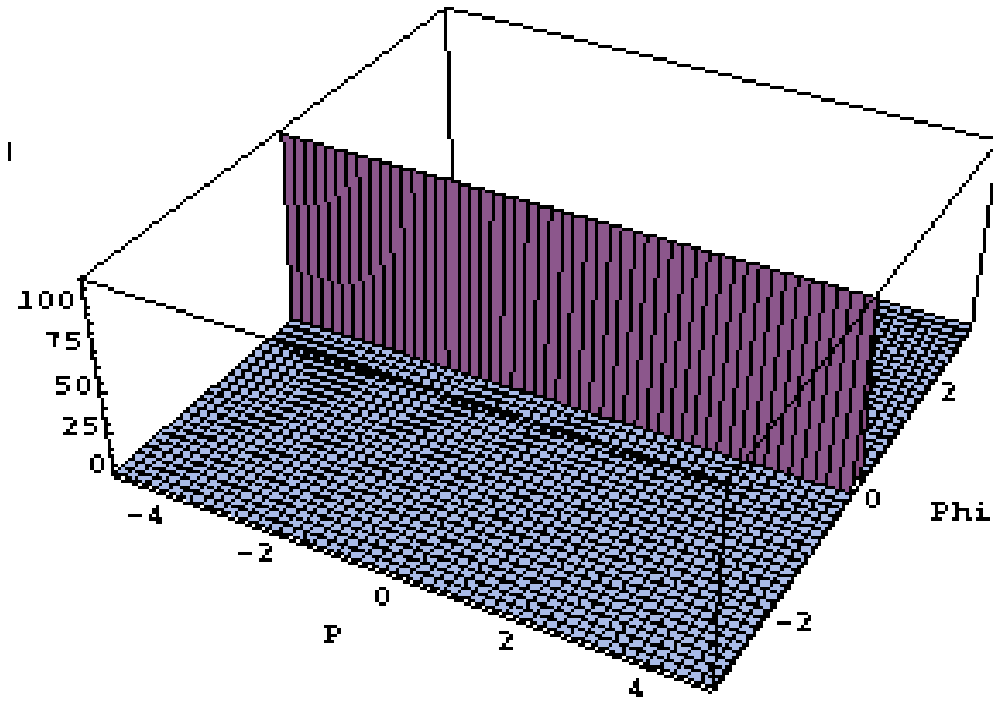

Figure 16: Probability amplitude $u \mapsto j_{g_{0}}^{t}(u)$ at $p \in[-5,5], h_{0}=1$.

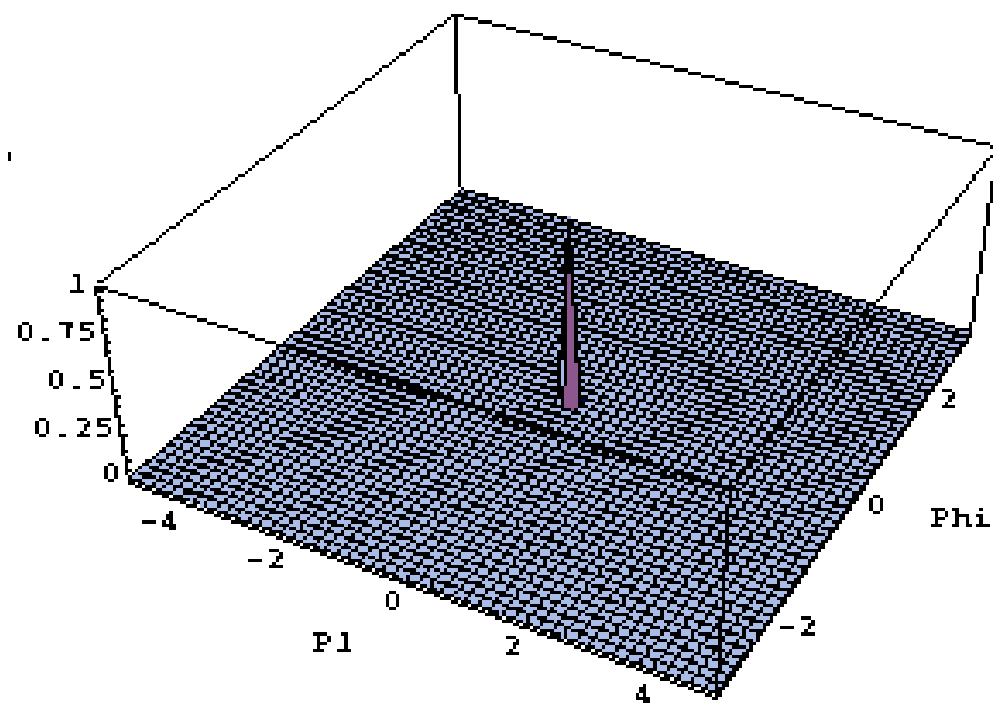

Figure 17: Overlap function $g \mapsto i_{g_{0}}^{t}(g)$ at $p=0, h_{0}=1$ for $p_{1} \in[-5,5], u \in U(1)$. 


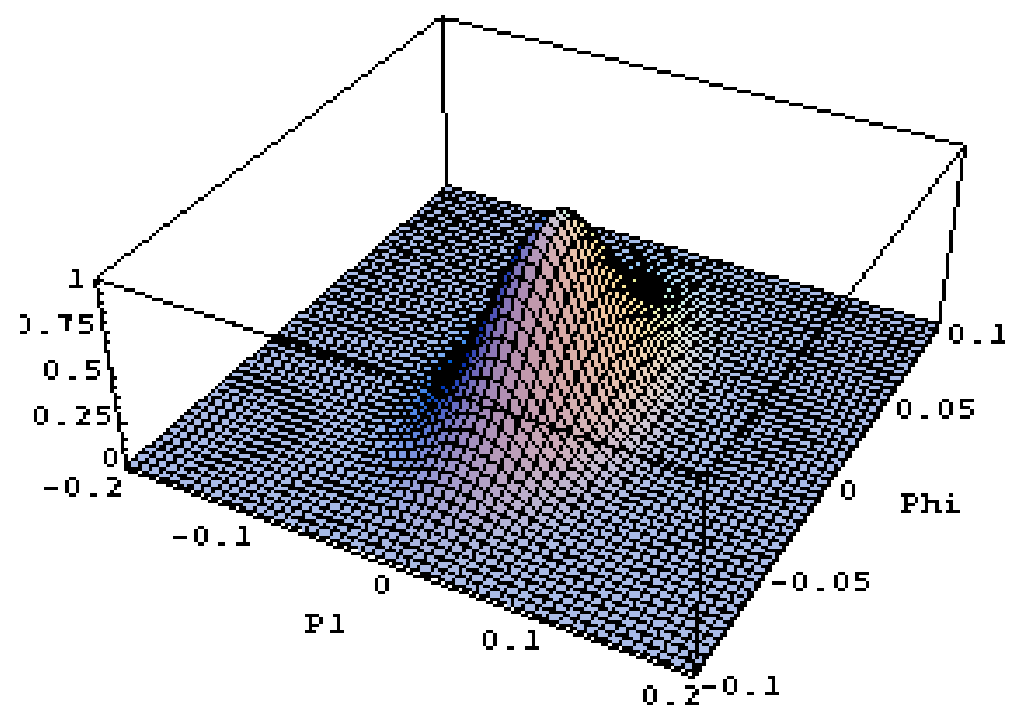

Figure 18: Resolution of a neighbourhood of the peak of the function $g \mapsto i_{g_{0}}^{t}(g)$ at $p=0, h_{0}=1$.

be highly background dependent. The best one can construct is a coherent state peaked on the Minkowski metric. The small fluctuations that are encoded in that state influence the propagation of matter and these tiny disturbances could accumulate to measurable sizes in so-called $\gamma$-ray burst experiments [78] where one measures the time delay of photons of higher energy as compared to those of lower energy as they travel over cosmological distances as a result of the energy dependence of the speed of light. If such an effect exists then it is a non-perturbative one because perturbatively defined QFT's on Minkowski space are by construction Poincaré invariant (recall e.g. the Wightman axioms from section [.1).

These are certainly only first moderate steps. The development of the semiclassical analysis for QGR is still in its very beginning and there are many interesting and new mathematical and physical issues that have to be settled before one can seriously attack the proof that, for instance, the Hamiltonian constraint of section [II.1 has the correct classcical limit or that full QGR reduces to classical GR plus the standard model in the low energy regime.

\section{III.6 Gravitons}

\section{III.6.1 The Isomorphism}

The reader with a strong background in ordinary QFT and/or string theory will have wondered throughout these lectures where in QGR the graviton, which plays such a prominent role in the perturbative, background dependent approaches to quantum gravity, resides. In fact, if one understands the graviton, as usually, as an excitation of the quantum metric around Minkowski space, then there is a clear connection with the semiclassical analysis of the previous section: One should construct a suitable coherent state which is peaked on the gauge invariant phase space point characterizing

and the fact that the Poincaré group should be a tiny subgroup thereof. However, this is not the case because we require the states only to be invariant under diffeomorphisms which are pure gauge and those have to die off at spatial infinity. Poincaré transformations are therefore not gauge transformation but symmetries and what we are saying is that there are no Poincaré symmetric, diffeomorphism gauge invariant states. 
Minkowski space and identify suitable excitations thereof as gravitons. It is clear that at the moment such graviton states from full QGR cannot be constructed, because we would need first to solve the Hamiltonian constraint.

However, one can arrive at an approximate notion of gravitons through the quantization of linearized gravity: Linearized gravity is nothing else than the expansion of the full GR action around the gauge variant initial data $\left(E^{0}\right)_{j}^{a}=\delta_{j}^{a},\left(A^{0}\right)_{a}^{j}=0$ to second order in $E-E^{0}, A$ which results in a free, classical field theory with constraints. In fact, the usual notion of gravitons is precisely the ordinary Fock space quantization of that classical, free field theory [79]. In order to see whether QGR can possibly accomodate these graviton states, Varadarajan in a beautiful series of papers 80 has carried out a polymer like quantization of that free field theory on a Hilbert space $\mathcal{H}_{\text {kin }}$ which is in complete analogy to that for full QGR, the only difference being that the gauge group $S U(2)$ is replaced by the gauge group $U(1)^{3}$. While there are certainly large differences between the highly interacting QGR theory and linearized gravity, one should at least be able to gain some insight into the the answer to the question, how a Hilbert space in which the excitations are one dimensional can possibly describe the Fock space excitations (which are three dimensional).

The problem of describing gravitons within linearized gravity by polymer like excitations is mathematically equivalent to the simpler problem of describing the photons of the ordinary Fock Hilbert space $\mathcal{H}_{F}$ of Maxwell theory by polymer like excitations within a Hilbert space $\mathcal{H}_{P}=L_{2}(\overline{\mathcal{A}}, d \mu)$ where $\overline{\mathcal{A}}$ is again a space of generalized $U(1)$ connections with some measure $\mu$ thereon. Thus, we decribe the latter problem in some detail since it requires less space and has the same educational value.

The crucial observation is the following isomorphism $\mathcal{I}$ between two different Poisson subalgebras of the Poisson algebra on the phase space $\mathcal{M}$ of Maxwell theory coordinatized by a canonical pair $(E, A)$ defined by a $U(1)$ connection $A$ and a conjugate electric field $E$ : Consider a one-parameter family of test functions of rapid decrease which are regularizations of the $\delta$-distribution, for instance

$$
f_{r}(x, y)=\frac{e^{-\frac{\|x-y\|^{2}}{2 r^{2}}}}{(\sqrt{2 \pi} r)^{3}}
$$

where we have made use of the Euclidean spatial background metric. Given a path $p \in \mathcal{P}$ we denote its distributional form factor by

$$
X_{p}^{a}(x):=\int_{0}^{1} d t \dot{p}^{a}(t) \delta(x, p(t))
$$

The smeared form factor is defined by

$$
X_{p, r}^{a}(x):=\int d^{3} y f_{r}(x, y) X_{p}^{a}(y)=\int_{0}^{1} d t \dot{p}^{a}(t) f_{r}(x, p(t))
$$

which is evidently a test function of rapid decrease. Notice that a $U(1)$ holonomy maybe written as

$$
A(p):=e^{i \int d^{3} x X_{p}^{a}(x) A_{a}(x)}
$$

and we can define a smeared holonomy by

$$
A_{r}(p):=e^{i \int d^{3} x X_{p, r}^{a}(x) A_{a}(x)}
$$


Likewise we may define smeared electric fields as

$$
E_{r}^{a}(x):=\int d^{3} y f_{r}(x, y) E^{a}(y)
$$

If we denote by $q$ the electric charge (notice that in our notation $\alpha=\hbar q^{2}$ is the fine structure constant), then we obtain the following Poisson subalgebras: On the one hand we have smeared holonomies but unsmeared electric fields with

$$
\left\{A_{r}(p), A_{r}\left(p^{\prime}\right)\right\}=\left\{E^{a}(x), E^{b}(y)\right\}=0, \quad\left\{E^{a}(x), A_{r}(p)\right\}=i q^{2} X_{p, r}^{a}(x) A_{r}(p)
$$

and on the other hand we have unsmeared holonomies but smeared electric fields with

$$
\left\{A(p), A\left(p^{\prime}\right)\right\}=\left\{E_{r}^{a}(x), E_{r}^{b}(y\}=0, \quad\left\{E_{r}^{a}(x), A(p)\right\}=i q^{2} X_{p, r}^{a}(x) h_{p}\right.
$$

Thus the two Poisson algebras are isomorphic and also the * relations are isomorphic, both $E^{a}(x), E_{r}^{a}(x)$ are real valued while both $A(p), A_{r}(p)$ are $U(1)$ valued. Thus, as abstract ${ }^{*}$ - Poisson algebras these two algebras are indistinguishable and we may ask if we can find different representations of it. Even better, notice that $A_{r}(p) A_{r}\left(p^{\prime}\right)=A_{r}\left(p \circ p^{\prime}\right), A_{r}(p)^{-1}=A_{r}\left(p^{-1}\right)$ so the smeared holonomy algebra is also isomorphic to the unsmeared one. Hence there is an algebra ${ }^{*}$-isomorphism $\mathcal{I}$ defined on the generators by $\mathcal{I}_{r}\left(h_{p}\right)=h_{p, r}, \mathcal{I}_{r}\left(E_{r}\right)=E$. One must also show that the $A_{r}(p)$ are still algebarically independent as are the $A(p)$ 80.

\section{III.6.2 Induced Fock Representation With Polymer - Excitations}

Now we know that the unsmeared holonmy algebra is well represented on the Hilbert space $\mathcal{H}_{\text {kin }}=$ $L_{2}\left(\overline{\mathcal{A}}, d \mu_{0}\right)$ while the smeared holonomy algebra is well represented on the Fock Hilbert space $\mathcal{H}_{F}=$ $L_{2}\left(\mathcal{S}^{\prime}, d \mu_{F}\right)$ where $\mathcal{S}^{\prime}$ denotes the space of divergence free, tempered distributions and $\mu_{F}$ is the Maxwell-Fock measure. These measures are completely characterized by their generating functional

$$
\omega_{F}\left(\hat{A}_{r}(p)\right):=\mu_{F}\left(A_{r}(p)\right)=e^{-\frac{1}{4 \alpha} \int d^{3} x X_{p, r}^{a}(x) \sqrt{-\Delta}^{-1} X_{p, r}^{b} \delta_{a b}}
$$

since finite linear combinations of the $h_{p, r}$ are dense in $\mathcal{H}_{F}$ 80. Here $\Delta=\delta^{a b} \partial_{a} \partial_{b}$ denotes the Laplacian and we have taken a loop $p$ rather than an open path so that $X_{p, r}$ is transversal. Also unsmeared electric fields are represented through the Fock state $\omega_{F}$ by

$$
\omega_{F}\left(\hat{A}_{r}(p) \hat{E}^{a}(x) \hat{A}_{r}\left(p^{\prime}\right)\right)=-\frac{\alpha}{2}\left[X_{p, r}^{a}(x)-X_{p^{\prime}, r}^{a}(x)\right] \omega_{F}\left(\hat{h}_{p \circ p^{\prime}, r}\right)
$$

and any other expectation value follows from these and the commutation relations.

Since $\omega_{F}$ defines a positive linear functional we may define a new representation of the algebra $A(p), E_{r}^{a}$ by

$$
\omega_{r}(\hat{A}(p)):=\omega_{F}\left(\hat{A}_{r}(p)\right) \text { and } \omega_{r}\left(\hat{A}(p) \hat{E}_{r}^{a}(x) \hat{A}\left(p^{\prime}\right)\right):=\omega_{F}\left(\hat{A}_{r}(p) \hat{E}^{a}(x) \hat{A}_{r}\left(p^{\prime}\right)\right)
$$

called the $r$-Fock representation. In other words, we have $\omega_{r}=\omega_{F} \circ \mathcal{I}_{r}$.

Since $\omega_{r}$ is a positive linear functional on $C(\overline{\mathcal{A}})$ by construction there exists is a measure $\mu_{r}$ on $\overline{\mathcal{A}}$ that represents $\omega_{r}$ in the sense of the Riesz representation theorem (recall [1.1.2.12]). In [81] Velhinho showed that the one - parameter family of measures $\mu_{r}$ are expectedly mutually singular with respect to each other and with respect to the uniform measure $\mu_{0}$ (that is, the support of one measure is a measure zero set with respect to the other and vice versa). 
Result 1: There is a unitary transformation between any of the Hilbert spaces $\mathcal{H}_{r}$ and their images under $\mathcal{I}_{r}$ in the usual Fock space $\mathcal{H}_{F}$. Since finite linear combinations of the $A_{r}(p)$ for fixed $r$ are still dense in $\mathcal{H}_{F}$ [80], there exists indeed a polymer like description of the usual $n$-photon states.

Recall that the Fock vacuum $\Omega_{F}$ is defined to be the zero eigenvalue coherent state, that is, it is annihilated by the annihilation operators

$$
\hat{a}(f):=\frac{1}{\sqrt{2 \alpha}} \int d^{3} x f^{a}\left[\sqrt[4]{-\Delta} \hat{A}_{a}-i(\sqrt[4]{-\Delta})^{-1} \hat{E}^{a}\right]
$$

where $f^{a}$ is any transversal smearing field. We then have in fact that $\omega_{F}()=.<\Omega_{F}, \Omega_{F}>_{\mathcal{H}_{F}}$. (For readers familiar with $C^{*}$-algebras this means that $\Omega_{F}$ is the cyclic vector that is determined by $\omega_{F}$ through the GNS construction.) The idea is now the following: From ([II.6.2.3) we see that we can easily answer any question in the $r$-Fock representation which has a preimage in the Fock representation, we just have to replace everywhere $A_{r}(p), E^{a}(x)$ by $A(p), E_{r}^{a}(x)$. Since in the $r-$ Fock representations only exponentials of connections are defined, we should exponentiate the annihilation operators and select the Fock vacuum through the condition

$$
e^{i \hat{a}(f)} \Omega_{F}=\Omega_{F}
$$

In particular, choosing $f=\sqrt{2 \alpha}(\sqrt[4]{-\Delta})^{-1} X_{p, r}$ for some loop $p$ we get

$$
e^{\int d^{3} x X_{p, r}^{a}\left[i \hat{A}_{a}+(\sqrt{-\Delta})^{-1} \hat{E}^{a}\right]} \Omega_{F}=\Omega_{F}
$$

Using the commutation relations and the Baker - Campell - Hausdorff formula one can write (III.6.2.6) in terms of $\hat{A}_{r}(p)$ and the exponential of the electric field appearing in (III.6.2.6) times a numerical factor. The resulting expression can then be translated into the $r$-Fock representation. Denoting the translated expression by $\mathcal{I}_{r}^{-1}\left(e^{i \hat{a}(f)}\right)$ we now ask the question, whether there exists a state $\Omega_{r} \in \mathcal{H}_{\text {kin }}=L_{2}\left(\overline{\mathcal{A}}, d \mu_{0}\right)$ such that $\mathcal{I}_{r}^{-1}\left(e^{i \hat{a}(f)}\right) \Omega_{r}=\Omega_{r}$. Remarkably, expanding $\Omega_{r}$ into the charge network basis introduced in section (III.1) one finds a (up to a multiplicative constant) unique solution given by

$$
\Omega_{r}=\sum_{c} e^{-\frac{\alpha}{2} \sum_{e, e^{\prime} \in E(\gamma(c))} G_{e, e^{\prime}}^{r} n_{e}(c) n_{e^{\prime}}(c)} \overline{T_{c}}
$$

where $c=\left(\gamma(c),\left\{n_{e}(c)\right\}_{e \in E(\gamma(c))}\right)$ denotes a charge network (the $U(1)$ analogue of a spin network) and

$$
G_{e, e^{\prime}}^{r}=\int d^{3} x X_{e, r}^{a} \sqrt{-\Delta}^{-1} X_{e^{\prime}, r}^{b} \delta_{a b}^{T}
$$

where $\delta_{a b}^{T}=\delta_{a b}-\partial_{a} \Delta^{-1} \partial_{b}$ denotes the transverse projector.

\section{Exercise III.6.1.}

Fill in the gaps that lead from (III.6.2.0 to (III.6.2.8).

Let us discuss this result. First of all, (III.6.2.7) is not normalizable with respect to the inner product on $\mathcal{H}_{\text {kin }}$ and neither are the images of $n$-photon states or coherent states from $\mathcal{H}_{F}$. This seems to indicate that the space $\mathcal{H}_{\text {kin }}$ does not play any role for physically interesting states. However, in [74] it was shown that this is not the case: It turns out, that, given a suitable regularization, that one can indeed obtain the expectation values such as $\omega_{r}(A(p))$ from the formal expression

$$
\omega_{r}(A(p)):=\frac{<\Omega_{r}, A(p) \Omega_{r}>}{\left\|\Omega_{r}\right\|^{2}}
$$


where both numerator and denominator are infinite but the fraction is finite.

Result 2: The polymer images of photon states can be obtained as certain limits of states from $\mathcal{H}_{\text {kin }}$ which therefore is a valid starting point in order to obtain physically interesting representations.

Moreover, as can be expected from the similarity between the formulas (III.6.2.8) and (III.5.2.4) (for $A^{\mathbb{C}}=0$ corresponding to vacuum $E=A=0$ in the present case), the states $\Omega_{r}$ also arise from a complexifier, given in this case by

$$
C=\frac{1}{2 q^{2}} \int_{\mathbb{R}^{3}} d^{3} x\left[E_{r}^{a} \sqrt{-\Delta}^{-1} E_{r}^{b}\right] \delta_{a b}
$$

Result 3: The complexifier framework is also able to derive images of $n$-photon states and usual Fock coherent states from the universal input of a complexifier.

We conclude that at least for the linearized theory the question posed at the beginning of this section could be answered affirmatively: There is indeed a precise framework available for how to accomodate graviton states into the framework of loop quantum gravity. This is a promising result and should have an analog in the full theory. 


\section{Part IV}

\section{Selection of Open Research Problems}


Let us summarize the most important open research problems that have come up during the discussion in these lectures.

i) Hamiltonian Constraint and Semiclassical States

The unsettled correctness of the quantum dynamics is the major roadblock to completing the quantization programme of QGR. In order to make progress a better understanding of the kinematical semiclassical sector of the theory is necessary.

ii) Physical Inner Product

Even if we had the correct Hamiltonian constraint and the complete space of solutions, at the moment there is no really good idea available of how to construct a corresponding physical inner product because the constraint algebra is not a Lie algebra but an open algebra in the BRST sense so that techniques from rigged Hilbert spaces are not available. A framework for such open algebras must be developed so that an inner product can be constructed at least in principle.

iii) Dirac Observables

Not even in classical general relativity do we know enough Dirac observables. For QGR they are mandatory for instance in order to select an inner product by adjointness conditions and in order to arrive at an interpretation of the final theory. A framework of how to define Dirac observables, at least in principle, even at the classical level, would be an extremely important contribution.

iv) Covariant Formulation

The connection between the Hamiltonian and the Spin Foam formulation is poorly understood. Without such a connection e.g. a proof of covariance of the canonical formulation on the one hand and a proof for the correct classical limit of the spin foam formulation on the other cannot be obtained using the respective other formulation. One should prove a rigorous Feynman Kac like formula that allows to switch between these complementary descriptions.

v) QFT on CST's and Hawking Effect from First Principles

The low energy limit of the theory in connection with the the construction of semiclassical states must be better understood. Once this is done, fundamental issues such as whether the Hawking effect is merely an artefact of an invalid description by QFT's on CST's while a quantum theory of gravity should be used or whether it is a robust result can be answered. Similar remarks apply to the information paradoxon associated with black holes etc.

vi) Combinatorial Formulation of the Theory

The description of a theory in terms of smooth and even analytic structures curves, surfaces etc. at all scales in which the spectra of geometrical operators are discrete at Planck scales is awkward and cannot be the most adequate language. There should be a purely combinatorical formulation in which notions such as topology, differential structure etc. can only have a semiclassical meaning.

vii) Avoidance of Classical and UV Singularities

That certain classical singularities are absent in loop quantum cosmology and that certain operators come out finite in the full theory while in the usual perturbative formulation they would suffer from UV singularities are promising results, but they must be better understood. If one could make contact with perturbative formulations and pin - point exactly why in QGR 
the usual perturbative UV singularities are absent then the theory would gain a lot more respect in other communities of high energy physicists. There must be some analog of the renormalization group and the running of coupling constants that one usually finds in QFT's and CST's. Similar remarks apply to the generalization of the loop quantum cosmology result to the full theory.

viii) Contact with String $(M)$ - Theory

If there is any valid perturbative description of quantum gravity then it is almost certainly string theory. It is conceivable that both string theory and loop quantum gravity are complementary descriptions but by themselves incomplete and that only a fusion of both can reach the status of a fundamental theory. To explore these possibilities, Smolin has launched an ambitious programme [82] which to our mind so far did not raise the interest that it deserves 18 . The contact arises through Chern - Simons theory which is part of both Loop Quantum Gravity and M - Theory 83] (when considered as the high energy limit of 11 dimensional Supergravity). Another obvious starting point is the definition of $\mathrm{M}$ - Theory as the quantum supermembrane in 11 dimensions [84, a theory that could be obtained as the quantization of the classical supermembrane by our non-perturbative methods. Finally, a maybe even more obvious connection could be found through the so-called Pohlmeyer String 85] which appears to be a method to quantize the string non-perturbatively, without supersymmetry, anomalies or extra dimensions, by working directly at the level of Dirac observables which are indeed possible to construct explicitly in this case.

We hope to have convinced the reader that Loop Quantum Gravity is an active and lively approach to a quantum theory of gravity which has produced already many non-trivial results and will continue to do so in the future. There are still a huge number of hard but fascinating problems to be solved of which the above list is at most the tip of an iceberg. If at least a tiny fraction of the readers would decide to dive into this challenging area and help in this endeavour, then these lectures would have been successful.

\section{Acknowledgements}

We thank the Heraeus - Stiftung and the organizers, Dominico Giulini, Claus Kiefer and Claus Lämmerzahl, for making this wonderful and successful meeting possible and the participants for creating a stimulating atmosphere through long and deep discussions, very often until early in the morning in the "Bürgerkeller".

\footnotetext{
${ }^{18}$ That we did not devote a section to this topic in this review is due to the fact that we would need to include an introduction to $\mathrm{M}$ - Theory into these lectures which would require too much space. The interested reader is referred to the literature cited.
} 


\section{Bibliography}

[1] T. Thiemann, "Introduction to Modern Canonical Quantum General Relativity", gr-qc/0110034

[2] C. Rovelli, "Loop Quantum Gravity", Review written for electronic journal "Living Reviews", gr-qc/9710008; "Strings, Loops and Others : a critical Survey of the present Approaches to Quantum Gravity", plenary lecture given at 15th Intl. Conf. on Gen. Rel. and Gravitation (GR15), Pune, India, Dec 16-21, 1997, gr-qc/9803024; "Notes for a Brief History of Quantum Gravity", gr-qc/0006061

M. Gaul, C. Rovelli, "Loop Quantum Gravity and the Meaning of Diffeomorphism Invariance", Lect.Notes Phys.541:277-324,2000, gr-qc/9910079

G. Horowitz, "Quantum Gravity at the Turn of the Millenium", gr-qc/0011089

S. Carlip, "Quantum Gravity: A Progress Report", Rept.Prog.Phys.64:885,2001 gr-qc/0108040

A. Ashtekar, "Quantum Mechanics of Geometry", gr-qc/9901023

[3] E. E. Flanagan, R. M. Wald, "Does Backreaction enforce the averaged null energy condition in semiclassical gravity ?", Phys. Rev. D54 (1996) 6233-6283, gr-qc/9602052

[4] R. M. Wald, "General Relativity", The University of Chicago Press, Chicago, 1989

[5] W. Junker, "Hadamard States, Adiabatic Vacua and the Construction of Physical States for Scalar Quantum Fields on Curved Spacetime", Rev. Math. Phys. 8 (1996) 1091 - 1159

[6] M.H. Goroff, A. Sagnotti, Phys. Lett. B160 (1985) 81 M.H. Goroff, A. Sagnotti, Nucl. Phys. B266 (1986) 709

[7] S. Deser, "Non-Renormalizability of (Last Hope) $D=11$ Supergravity with a Survey of Divergences in Quantum Gravities", hep-th/9905017

[8] O. Lauscher, M. Reuter, "Towards Nonperturbative Renormalizability of Quantum Einstein Gravity", Int. J. Mod. Phys. A17 993 (2002), hep-th/0112089]; "Is Quantum Gravity Nonperturbatively Renormalizable ?", Class. Quant. Grav. 19483 (2002), hep-th/0110021

[9] J. Polchinsky, "String Theory", vol. 1 and 2, Cambridge University Press, Cambridge, 1998

[10] D. Marolf, C. Rovelli, "Relativistic Quantum Measurement", Phys.Rev. D66 (2002) 023510, gr-qc/0203056

[11] J. B. Hartle, S. W. Hawking, Phys. Rev. D28 (1983) 2960

[12] J. Ambjorn, J. Jurkiewicz, R. Loll, "Lorentzian and Euclidean Quantum Gravity: Analytical and Numerical Results", hep-th/0001124; "A Non-Perturbative Lorentzian Path Integral for Gravity", Phys. Rev. Lett. 85 (2000) 924, hep-th/0002050

J. Ambjorn, A. Dasgupta, J. Jurkiewicz, R. Loll, "A Lorentzian Cure For Euclidean Troubles", Nucl. Phys. Proc. Suppl. 106 (2002) 977-979, hep-th/0201104

R. Loll, A. Dasgupta, "A Proper Time Cure for the Conformal Sickness in Quantum Gravity", Nucl. Phys. B606 (2001) 357-379, hep-th/0103186

[13] M. J. Gotay, J. Isenberg, J. E. Marsden, "Momentum Maps and Classical Relativistic Fields. Part 1: Covariant Field Theory", (with the collaboration of Richard Montgomery, Jedrzej Sniatycki and Philip B. Yasskin) physics/9801019

H.A. Kastrup, "Canonical Theories of Dynamical Systems in Physics" Phys. Rept. 101 (1983) 
I. V. Kanatchikov, "Canonical Structure of Classical Field Theory in the Polymomentum Phase Space", Rept. Math. Phys. 41 (1998) 49-90, hep-th/9709229; "On the Field Theoretic Generalizations of a Poisson Algebra", Rept. Math. Phys. 40 (1997) 225, hep-th/9710069

C. Rovelli, "A Note on the Foundation of Relativistic Mechanics" gr-qc/0111037; ". 2. Covariant Hamiltonian General Relativity", gr-qc/0202079]; "Covariant Hamiltonian Formalism for Field Theory: Symplectic Structure and Hamilton-Jacobi Equation on the Space G", grc/0207043

[14] A. Ashtekar, L. Bombelli, O. Reula, "The Covariant Phase Space of Asymptotically Flat Gravitational Fields", in: "Analysis, Geometry and Mechanics: 200 Years After Lagrange", Ed. by M. Francaviglia, D. Holm, North-Holland, Amsterdam, 1991

[15] R.E. Peierls, Proc. R. Soc. Lond. A 214 (1952) 143

B. S. DeWitt, "Dynamical Theory of Groups and Fields", Gordon \& Breach, New York, 1965

[16] C. J. Isham, N. Linden, "Quantum Temporal Logic and Decoherence Functionals in the Histories Approach to Generalized Quantum Theory", J. Math. Phys. 35 (1994) 5452-5476 [grqc/9405029|; "Continuous Histories and the History Group in generalized Quantum Theory", J. Math. Phys. 36 (1995) 5392-5408, gr-qc/9503063

C.J. Isham, N. Linden, K. Savvidou, S. Schreckenberg, "Continuous Time and Consistent Histories" J. Math. Phys. 39 (1998) 1818-1834, quant-ph/9711031

[17] R. B. Griffiths, J. Stat. Phys. 36 (1984) 219; Found. Phys. 231601

R. Omnés, J. Stat. Phys. 53 (1988) 893; ibid 53 (1988) 933; ibid 53 (1988) 957; ibid 57 (1989) 357; Rev. Mod. Phys. 64 (1992) 339

M. Gell-Mann , J. B. Hartle, "Classical Equations for Quantum Systems", Phys. Rev. D47 (1993) 3345-3382, gr-qc/9210010; " Equivalent Sets of Histories and Multiple Quasiclassical Domains", gr-qc/9404013; "Strong Decoherence", gr-qc/9509054

J. B. Hartle, "Space-Time Quantum Mechanics and the Quantum Mechanics of Space-Time", Les Houches Sum.Sch.1992:0285-480, gr-qc/9304006

F. Dowker, A. Kent, "Properties of Consistent Histories" Phys.Rev.Lett. 75 (1995) 3038-3041, [gr-qc/9409037; "On the Consistent Histories Approach to Quantum Mechanics" J. Statist. Phys. 82 (1996) 1575-1646, gr-qc/9412067]

[18] I. V. Kanatchikov, "Precanonical Perspective in Quantum Gravity", Nucl. Phys. Proc. Suppl. 88 (2000) 326-330, gr-qc/0004066; "Precanonical Quantization and the Schroedinger Wave Functional", Phys. Lett. A283 (2001) 25-36 hep-th/0012084]; "Precanonical Quantum Gravity: Quantization without the Spacetime Decomposition", Int. J. Theor. Phys. 40 (2001) 1121-1149, gr-qc/0012074

[19] C.J. Isham, K.N. Savvidou, "Quantizing the Foliation in History Quantum Field Theory", quant-ph/0110161

[20] P. A. M. Dirac, "Lectures on Quantum Mechanics", Belfer Graduate School of Science, Yeshiva University Press, New York, 1964

[21] D. M. Gitman, I. V. Tyutin, "Quantization of Fields with Constraints", Springer-Verlag, Berlin, 1990

M. Henneaux, C. Teitelboim, "Quantization of Gauge Systems" Princeton University Press, Princeton, 1992

[22] P. G. Bergmann, A. Komar, "The Phase Space Formulation of General Relativity and Approaches Towards its Canonical Quantization", Gen. Rel. Grav., 1 (1981) 227-254

A. Komar, "General Relativistic Observables via Hamilton Jacobi Functionals", Phys. Rev. D4 (1971) 923-927; "Commutator Algebra of General Relativistic Observables", Phys. Rev. D9 
(1974) 885-888; "Generalized Constraint Structure for Gravitation Theory", Phys. Rev. D27 (1983) 2277-2281; "Consistent Factor Ordering of General Relativistic Constraints", Phys. Rev. D20 (1979) 830-833

P. G. Bergmann, A. Komar, "The Coordinate Group Symmetries of General Relativity", Int. J. Theor. Phys. 5 (1972) 15

[23] S. A. Hojman, K. Kuchar, C. Teitelboim, "Geometrodynamics Regained", Annals Phys. 96 (1976) 88-135

[24] C.G. Torre, I.M. Anderson, "Symmetries of the Einstein Equations" Phys. Rev. Lett. 70 (1993) 3525-3529, gr-qc/9302033]; "Classification of Generalized Symmetries for the Vacuum Einstein Equations", Commun. Math. Phys. 176 (1996) 479-539, gr-qc/9404030

[25] A. Ashtekar, Phys. Rev. Lett. 57 (1986) 2244, Phys. Rev. D36 (1987) 1587

[26] G. Immirzi, Nucl. Phys. Proc. Suppl. 57 (1997) 65

[27] F. Barbero, Phys. Rev. D51 (1995) 5507; Phys. Rev. D51 (1995) 5498

[28] M. Nakahara, "Geometry, Topology and Physics", Institute of Physics Publishing, Bristol, 1998

[29] D. Giulini, D. Marolf, "On the Generality of Refined Algebraic Quantization", Class. Quant. Grav. 16 (1999) 2479-2488 gr-qc/9812024

[30] N. M. J. Woodhouse, "Geometric Quantization", 2nd. edition, Clarendon Press, Oxford, 1991

[31] R. Gambini, A. Trias, Phys. Rev. D22 (1980) 1380

C. Di Bartolo, F. Nori, R. Gambini, A. Trias, Lett. Nuov. Cim. 38 (1983) 497

R. Gambini, A. Trias, Nucl. Phys. B278 (1986) 436

[32] M. F. Atiyah, "Topological Quantum Field Theories", Publ. Math. IHES 68 (1989) 175-186

M. F. Atiyah, "The Geometry of Physics and Knots", Cambridge University Press, Cambridge 1990

[33] T. Jacobson, L. Smolin, "Nonperturbative Quantum Geometries" Nucl. Phys. B299 (1988) 295, C. Rovelli, L. Smolin, "Loop Space Representation of Quantum General Relativity", Nucl. Phys. B331, 80 (1990)

[34] R. Giles, Phys. Rev. D8 (1981) 2160

[35] H. Sahlmann, "When Do Measures on the Space of Connections Support the Triad Operators of Loop Quantum Gravity", gr-qc/0207112; "Some Comments on the Representation Theory of the Algebra Underlying Loop Quantum Gravity", gr-qc/0207111

H. Sahlmann, T. Thiemann, "Representation Theory of Diffeomorphism Invariant Gauge Theories", in preparation

[36] A. Ashtekar and J. Lewandowski, J. Math. Phys. 36, 2170 (1995).

[37] J. Velhinho, "A Groupoid Approach to Spaces of Generalized Connections", hep-th/0011200

[38] A. Ashtekar, C. J. Isham, Class. Quantum Grav. 9 (1992) 1433

[39] A. Ashtekar and J. Lewandowski, "Representation theory of analytic holonomy $C^{\star}$ algebras", in Knots and quantum gravity, J. Baez (ed), Oxford University Press, Oxford 1994

[40] C. Rovelli, L. Smolin, "Spin Networks and Quantum Gravity", Phys. Rev. D53 (1995) 5743

J. Baez, "Spin Networks in Non-Perturbative Quantum Gravity", in : "The Interface of Knots and Physics", L. Kauffman (ed.), American Mathematical Society, Providence, Rhode Island, 1996, [gr-qc/9504036]

[41] N. J. Vilenkin, "Special Functions and the Theory of Group Representations", American Mathematical Society, Providence, Rhode Island, 1968

[42] T. Thiemann, O. Winkler, "Gauge Field Theory Coherent States (GCS): IV. Infinite Tensor Product and Thermodynamical Limit", Class. Quantum Grav. 18 (2001) 4997-5033, hepth/0005235

M. Arnsdorf, "Loop Quantum Gravity on Noncompact Spaces", Nucl. Phys. B577 (2000) 529546, gr-qc/9909053] 
[43] A. Ashtekar, J. Lewandowski, D. Marolf, J. Mourão, T. Thiemann, "Quantization for diffeomorphism invariant theories of connections with local degrees of freedom", Journ. Math. Phys. 36 (1995) 6456-6493, gr-qc/9504018]

[44] C. Rovelli, L. Smolin, "Discreteness of volume and area in quantum gravity", Nucl. Phys. B442 (1995) 593, Erratum : Nucl. Phys. B456 (1995) 734 A. Ashtekar, J. Lewandowski, "Quantum Theory of Geometry I: Area Operators", Class. Quantum Grav. 14 (1997) A55-81

[45] A. Ashtekar, J. Lewandowski, "Quantum Theory of Geometry II : Volume Operators", Adv. Theo. Math. Phys. 1 (1997) 388-429

J. Lewandowski, Class. Quantum Grav. 14 (1997) 71-76

R. Loll, "Spectrum of the Volume Operator in Quantum Gravity", Nucl.Phys.B460:143-154,1996 gr-qc/9511030

R. De Pietri, C. Rovelli, "Geometry Eigenvalues and Scalar Product from Recoupling Theory in Loop Quantum Gravity", Phys. Rev. D54 (1996) 2664, gr-qc/9602023

T. Thiemann, "Closed Formula for the Matrix Elements of the Volume Operator in Canonical Quantum Gravity", Journ. Math. Phys. 39 (1998) 3347-3371, gr-qc/9606091]

[46] T. Thiemann, "A Length Operator for Canonical Quantum Gravity", Journ. Math. Phys. 39 (1998) 3372-3392, gr-qc/9606092

[47] T. Thiemann, "Spatially Diffeomorphism Invariant Geometrical Operators in Quantum General Relativity", work in progress

[48] T. Thiemann, "Anomaly-free Formulation of non-perturbative, four-dimensional Lorentzian Quantum Gravity", Physics Letters B380 (1996) 257-264, gr-qc/9606088

T. Thiemann, "Quantum Spin Dynamics (QSD): I.", Class. Quantum Grav. 15 (1998) 839-73, gr-qc/9606089; "II. The Kernel of the Wheeler-DeWitt Constraint Operator", Class. Quantum Grav. 15 (1998) 875-905, gr-qc/9606090; "III. Quantum Constraint Algebra and Physical Scalar Product in Quantum General Relativity", Class. Quantum Grav. 15 (1998) 1207-1247, gr-qc/9705017]; "IV. 2+1 Euclidean Quantum Gravity as a model to test 3+1 Lorentzian Quantum Gravity", Class. Quantum Grav. 15 (1998) 1249-1280, gr-qc/9705018; "V. Quantum Gravity as the Natural Regulator of the Hamiltonian Constraint of Matter Quantum Field Theories", Class. Quantum Grav. 15 (1998) 1281-1314, gr-qc/9705019; "VI. Quantum Poincaré Algebra and a Quantum Positivity of Energy Theorem for Canonical Quantum Gravity", Class. Quantum Grav. 15 (1998) 1463-1485, [gr-qc/9705020]; "Kinematical Hilbert Spaces for Fermionic and Higgs Quantum Field Theories", Class. Quantum Grav. 15 (1998) 1487-1512, gr-qc/9705021

[49] H. Sahlmann, T. Thiemann, "Towards the QFT on Curved Spacetime Limit of QGR. 1. A General Scheme", gr-qc/0207030; “2. A Concrete Implementation", gr-qc/0207031

[50] D. Marolf, J. Lewandowski, "Loop Constraints : A Habitat and their Algebra", Int.J.Mod.Phys.D7:299-330,1998, gr-qc/9710016

R. Gambini, J. Lewandowski, D. Marolf, J. Pullin, "On the Consistency of the Constraint Algebra in Spin Network Gravity", Int.J.Mod.Phys.D7:97-109,1998, gr-qc/9710018

[51] M. Gaul, C. Rovelli, "A Generalized Hamiltonian Contraint Operator in Loop Quantum Gravity and its Simplest Euclidean Matrix Elements", Class.Quant.Grav.18:1593-1624,2001, grqc/0011106

[52] C. Kiefer, Lect.Notes Phys.541:158-187,2000, gr-qc/9906100 A.O. Barvinsky, "Quantum Cosmology at the Turn of the Millenium", gr-qc/0101046

J. B. Hartle, "Quantum Cosmology: Problems for the 21st Century", gr-qc/9701022

[53] M. Bojowald, "Loop Quantum Cosmology. I. Kinematics", Class. Quantum Grav. 17 (2000) 1489 gr-qc/9910103; "II. Volume Operators", Class. Quantum Grav. 17 (2000) 1509 gr-qc/9910104; "III. Wheeler-DeWitt Operators", Class.Quant.Grav.18:1055-1070,2001 
gr-qc/0008052; "IV. Discrete Time Evolution" Class.Quant.Grav.18:1071-1088,2001 grc/0008053; "Absence of Singularity in Loop Quantum Cosmology", Phys.Rev.Lett.86:52275230,2001, gr-qc/0102069; "Dynamical Initial Conditions in Quantum Cosmology", Phys.Rev.Lett.87:121301,2001, [gr-qc/0104072]; "The Inverse Scale Factor in Isotropic Quantum Geometry", Phys.Rev.D64:084018,2001, gr-qc/0105067]; "The Semiclassical Limit of Loop Quantum Cosmology", Class.Quant.Grav.18:L109-L116,2001, gr-qc/0105113; "Inflation from Quantum Geometry", gr-qc/0206054

[54] J. C. Baez , "An Introduction to Spin Foam Models of Quantum Gravity and BF Theory", Lect.Notes Phys.543:25-94,2000, gr-qc/9905087

J. C. Baez, "Spin Foam Models", Class.Quant.Grav.15:1827-1858,1998, gr-qc/9709052

J. W. Barrett, "State Sum Models for Quantum Gravity", gr-qc/0010050; "Quantum Gravity as Topological Quantum Field Theory", J.Math.Phys.36:6161-6179,1995, gr-qc/9506070

A. Perez, "Spin Foam Models for Quantum Gravity", to appear

[55] M. Reisenberger, C. Rovelli, "Sum over Surfaces Form of Loop Quantum Gravity", Phys. Rev. D56 (1997) 3490-3508

[56] G. Roepstorff, "Path Integral Approach to Quantum Physics: An Introduction", Springer Verlag, Berlin, 1994

[57] S. Holst, "Barbero's Hamiltonian Derived From a Generalized Hilbert-Palatini Action", Phys. Rev. D53 (1996) 5966, gr-qc/9511026

N. Barros e Sá, "Hamiltonian Analysis of General Relativity with the Immirzi Parameter", Int. J. Mod. Phys. D10 (2001) 261-272, gr-qc/0006013

[58] L. Freidel, K. Krasnov, R. Puzio, "BF Description of Higher Dimensional Gravity Theories", Adv. Theor. Math. Phys. 3 (1999) 1289-1324, hep-th/9901069]

[59] J. W. Barrett, L. Crane, "Relativistic Spin Networks and Quantum Gravity", J. Math. Phys. 39 (1998) 3296-3302, gr-qc/9709028]

[60] J. C. Baez, J. W. Barrett, "Integrability of Relativistic Spin Networks", gr-qc/0101107

A. Perez, C. Rovelli, "Spin Foam Model for Lorentzian General Relativity", Phys. Rev. D63 (2001) 041501, gr-qc/0009021

L. Crane, A. Perez, C. Rovelli, "A Finiteness Proof for the Lorentzian State Sum Spin Foam Model for Quantum General Relativity", gr-qc/0104057; "Perturbative Finiteness in Spin-Foam Quantum Gravity", Phys. Rev. Lett. 87 (2001) 181301

[61] D. V. Boulatov, Mod. Phys. Lett. A7 (1992) 1629

H. Ooguri, Mod. Phys. Lett. A7 (1992) 2799

[62] F. Markopoulou, L. Smolin, "Causal Evolution of Spin Networks", Nucl.Phys.B508:409430,1997, gr-qc/9702025

F. Markopoulou, "Dual Formulation of Spin Network Evolution", gr-qc/9704013

F. Markopoulou, L. Smolin, "Quantum Geometry with Intrinsic Local Causality", Phys.Rev.D58:084032,1998, gr-qc/9712067

F. Markopoulou, "The Internal Description of a Causal Set: What the Universe Like from Inside", Commun.Math.Phys.211:559-583,2000, gr-qc/9811053]

F. Markopoulou, "Quantum Causal Histories", Class.Quant.Grav.17:2059-2072,2000, hepth/9904009

F. Markopoulou, "An Insider's Guide to Quantum Causal Histories", Nucl.Phys.Proc.Suppl.88:308-313,2000, hhep-th/9912137

[63] F. Markopoulou, "An Algebraic Approach to Coarse Graining", hep-th/0006199

[64] J. C. Baez, J. D. Christensen, T. R. Halford, D. C. Tsang, "Spin Foam Models of Riemannian Quantum Gravity", Class. Quant. Grav. 19 (2002) 4627-4648, gr-qc/0202017 
A. Perez, "Spin Foam Quantization of Plebanski's Action", Adv. Theor. Math. Phys. 5 (2002) 947-968, gr-qc/0203058

[65] M. Bojowald and A. Perez, "Spin Foam Quantization and Anomalies", in preparation

[66] J. D. Bekenstein, "Black Holes and Entropy", Phys.Rev. D7 (1973) 2333-2346

J. D. Bekenstein, "Generalized Second Law for Thermodynamics in Black Hole Physics", Phys. Rev. D9 (1974) 3292-3300

S.W. Hawking, "Particle Creation by Black Holes", Commun. Math. Phys. 43 (1975) 199-220

[67] K. Krasnov, "On Statistical Mechanics of Gravitational Systems", Gen. Rel. Grav. 30 (1998) 53-68, gr-qc/9605047

C. Rovelli, "Black Hole Entropy from Loop Quantum Gravity" Phys. Rev. Lett. 77 (1996) 3288-3291, gr-qc/9603063

[68] A. Ashtekar, C. Beetle, O. Dreyer, S. Fairhurst, B. Krishnan, J. Lewandowski, J. Wisniewski "Isolated Horizons and Their Applications", Phys.Rev.Lett.85:3564-3567,2000 gr-qc/0006006

A. Ashtekar, "Classical and Quantum Physics of Isolated Horizons", Lect.Notes Phys.541:5070,2000

A. Ashtekar, "Interface of general Relativity, Quantum Physics and Statistical Mecahnics: Some recent Developments", Annalen Phys.9:178-198,2000, gr-qc/9910101.

[69] A. Ashtekar, A. Corichi, K. Krasnov, "Isolated Horizons: The Classical Phase Space", Adv.Theor.Math.Phys.3:419-478,2000, gr-qc/9905089

A. Ashtekar, J. C. Baez, K. Krasnov, "Quantum Geometry of Isolated Horizons and Black Hole Entropy", Adv.Theor.Math.Phys.4:1-94,2001, gr-qc/0005126

[70] T. Regge, C. Teitelboim, "Role of Surface Integrals in the Hamiltonian Formulation of General Relativity", Annals Phys. 88 (1974) 286

[71] L. Smolin, "Linking Topological Quantum Field Theory and Non-Perturbative Quantum Gravity", J. Math. Phys. 36 (1995) 6417, gr-qc/9505028

[72] S. Axelrod, S. D. Pietra, E. Witten, "Geometric Quantization of Chern-Simons Gauge Theory", J. Diff. Geo. 33 (1991) 787-902

[73] G. 't Hooft, "The Holographic Principle: Opening Lecture", in "Erice 1999, Basics and highlights in fundamental physics", 397-413, hep-th/0003004

[74] T. Thiemann, "Reality Conditions inducing Transforms for Quantum Gauge Field Theories and Quantum Gravity", Class. Quantum Gravity 13 (1996) 1383-1403, gr-qc/9511057; "An Account of Transforms on $\mathcal{A} / \mathcal{G}$ ", Acta Cosmologica 21 (1995) 145-167, gr-qc/9511049; "Gauge Field Theory Coherent States (GCS): I. General Properties", Class.Quant.Grav.18:20252064,2001, hep-th/0005233; "Complexifier Coherent States for Quantum General Relativity", gr-qc/0206037

[75] T. Thiemann, "Quantum Spin Dynamics (QSD): VII. Symplectic Structures and Continuum Lattice Formulations of Gauge Field Theories", Class.Quant.Grav.18:3293-3338,2001, [hepth/0005232

T. Thiemann, O. Winkler, "Gauge Field Theory Coherent States (GCS): II. Peakedness Properties", Class.Quant.Grav.18:2561-2636,2001, hep-th/0005237; "III. Ehrenfest Theorems", Class. Quantum Grav. 18 (2001) 4629-4681, hep-th/0005234

H. Sahlmann, T. Thiemann, O. Winkler, "Coherent States for Canonical Quantum General Relativity and the Infinite Tensor product Extension", Nucl.Phys.B606:401-440,2001, grqc/0102038

[76] B. C. Hall, Journ. Funct. Analysis 122 (1994) 103

B. C. Hall, J. J. Mitchell, "Coherent States on Spheres", J. Math. Phys. 43 (2002) 12111236, quant-ph/0109086; "The Large Radius Limit for Coherent States on Spheres", quantph/0203142 
[77] G. Amelino-Camelia, "Are we at Dawn with Quantum Gravity Phenomenology", Lectures given at 35th Winter School of Theoretical Physics: From Cosmology to Quantum Gravity, Polanica, Poland, 2-12 Feb 1999, Lect.Notes Phys.541:1-49,2000, gr-qc/9910089

G. Amelino-Camelia, John R. Ellis, N.E. Mavromatos, D.V. Nanopoulos, Subir Sarkar, "Potential Sensitivity of Gamma Ray Burster Observations to Wave Dispersion in Vacuo", Nature 393:763-765,1998, astro-ph/9712103 R. Gambini, J. Pullin, "Nonstandard Optics from Quantum Spacetime" Phys.Rev.D59:124021,1999, gr-qc/9809038]

R. Gambini, J. Pullin, "Quantum Gravity Experimental Physics ?", Gen.Rel.Grav.31:16311637,1999

J. Alfaro, H. A. Morales-Tecotl, L. F. Urrutia, "Quantum Gravity Corrections to Neutrino Propagation", Phys.Rev.Lett.84:2318-2321,2000, gr-qc/9909079

J. Alfaro, H. A. Morales-Tecotl, L. F. Urrutia, "Loop Quantum Gravity and Light Propagation", Phys. Rev. D65 (2002) 103509, [hep-th/0108061]

[78] S. D. Biller et al, Phys. Rev. Lett. 83 (1999) 2108

[79] A. Ashtekar, Carlo Rovelli, L. Smolin, "Gravitons and Loops", Phys.Rev. D44 (1991) 1740-1755, hep-th/9202054

[80] M. Varadarajan, "Fock representations from U(1) Holonomy Algebras", Phys. Rev. D61 (2000) 104001 gr-qc/0001050; "Photons from Quantized Electric Flux Representations", Phys.Rev. D64 (2001) 104003, [gr-qc/0104051]; M. Varadarajan, "Gravitons from a Loop Representation of Linearized Gravity", Phys. Rev. D66 (2002) 024017, grr-qc/0204067]

[81] J. Velhinho, "Invariance Properties of Induced Fock Measures for U(1) Holonomies", Commun. Math. Phys. 227 (2002) 541-550, math-ph/0107002

[82] L. Smolin, "Strings as Perturbations of Evolving Spin Networks", Nucl.Phys.Proc.Suppl.88:103113,2000, [hep-th/9801022]; "A Holographic Formulation of Quantum General Relativity", Phys.Rev.D61:084007,2000, hhep-th/9808191; "Towards a Background Independent Approach to M Theory", hep-th/9808192; "The Cubic Matrix Model and Duality Between Strings and Loops", hep-th/0006137; "A Candidate for a Background Independent Formulation of M Theory", Phys.Rev.D62:086001,2000, hhep-th/9903166]; "The Exceptional Jordan Algebra and the Matrix String", hep-th/0104050

Y. Ling, L. Smolin, "Eleven - Dimensional Supergravity as a Constrained Topological Field Theory", Nucl.Phys.B601:191-208,2001, hep-th/0003285]; "Supersymmetric Spin Networks and Quantum Supergravity", Phys.Rev.D61:044008,2000, [hep-th/9904016]; "Holographic Formulation of Quantum Supergravity", Phys.Rev.D63:064010,2001, hep-th/0009018]

[83] L. Smolin, "M Theory as a Matrix Extension of Chern-Simons Theory", Nucl.Phys.B591:227242,2000, hep-th/0002009; L. Smolin, "Quantum Gravity with a Positive Cosmological Constant", hep-th/0209079

[84] R. Helling, H. Nicolai, "Supermebranes and Matrix Theory", hep-th/9809103

[85] K. Pohlmeyer, "A Group Theoretical Approach to the Quantization of the Free Relativistic Closed String", Phys. Lett. B119 (1982) 100

K. Pohlmeyer, K.H. Rehren, "Algebraic Properties of the Invariant Charges of the Nambu-Goto Theory", Commun.Math.Phys. 105 (1986) 593; "The Algebra formed by the Charges of the Nambu-Goto Theory: Identification of a Maximal Abelean Subalgebra" Commun. Math. Phys. 114 (118) 55; "The Algebra formed by the Charges of the Nambu-Goto Theory: Their Geometric Origin and Their Completeness", Commun. Math. Phys. 114 (1988) 177

K. Pohlmeyer, "The Invariant Charges of the Nambu-Goto Theory in WKB Approximation", Commun. Math. Phys. 105 (1986) 629; "The Algebra formed by the Charges of the NambuGoto Theory: Casimir Elements", Commun. Math. Phys. 114 (1988) 351; "Uncovering the 
Detailed Structure of the Algebra Formed by the Invariant Charges of Closed Bosonic Strings Moving in (1+2)-Dimensional Minkowski Space", Commun. Math. Phys. 163 (1994) 629-644; "The Invariant Charges of the Nambu-Goto Theory: Non-Additive Composition Laws", Mod. Phys. Lett. A10 (1995) 295-308; "The Nambu-Goto Theory of Closed Bosonic Strings Moving in (1+3)-Dimensional Minkowski Space: The Quantum Algebra of Observables", Annalen Phys. 8 (1999) 19-50, [hep-th/9805057]

K. Pohlmeyer, M. Trunk, "The Invariant Charges of the Nambu-Goto Theory: Quantization of Non-Additive Composition Laws", hep-th/0206061

G. Handrich, C. Nowak, "The Nambu-Goto Theory of Closed Bosonic Strings Moving in (1+3)Dimensional Minkowski Space: The Construction of the Quantum Algebra of Observables up to Degree Five", Annalen Phys. 8 (1999) 51-54, hep-th/9807231]

G. Handrich, "Lorentz Covariance of the Quantum Algebra of Observables: Nambu-Goto Strings in 3+1 Dimensions", Int. J. Mod. Phys. A17 (2002) 2331-2349

G. Handrich, C. Paufler, J.B. Tausk, M. Walter, "The Representation of the Algebra of Observables of the Closed Bosonic String in 1+3 Dimensions: Calculation to Order $\hbar^{7}$ ", mathh/0210024

C. Meusburger, K.H. Rehren, "Algebraic Quantization of the Closed Bosonic String", mathh/0202041. 